Universidad deValladolid

PROGRAMA DE DOCTORADO EN CONSERVACIÓN Y USO SOSTENIBLE DE LOS SISTEMAS FORESTALES

DOCTORAL THESIS:

\title{
Spatial capture-recapture models for the ecological study of common voles (Microtus arvalis) in NW Spain
}

\author{
presented by Juan Romairone \\ A thesis submitted to attain the degree of \\ Doctor by the University of Valladolid
}

Advisors:

Dr. Juan José Luque Larena

Dr. François R. Mougeot

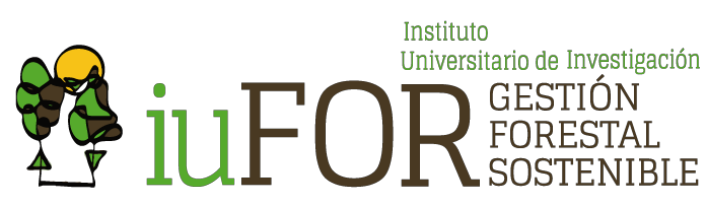


"El verdadero conservacionista es el que sabe que el mundo no es una herencia de sus padres, sino un préstamo de sus hijos"

J.J.Audubon

A la educación recibida.

A mi familia. 
Abstract 1

Resumen $\quad 4$

$\begin{array}{ll}\text { 1. Introduction } & 7\end{array}$

1.1 Monitoring small mammal populations 8

1.1.1 Obtaining reliable estimates and general applications of 8 abundance variations

1.1.2 The study of small mammals: elusive and cryptic species that require specific methodology for monitorization

1.2 Different methods to monitor the abundance of rodent populations

1.2.1 Direct methods based on capture-mark-recapture (CMR) 15

1.2.2 Direct method based on a single catch effort (Single Trapping Sessions; STS)

1.2.3 Indirect method based on Vole Activity Signs (VAS)

1.3 Statistical analysis of capture-recapture data

20

1.3.1 Non-spatial analysis of capture-recapture (CR) data 21

1.3.2 Spatial analysis of capture-recapture data 22

1.4 A case study on common voles (Microtus arvalis) 24

2. Objectives 26

3. Chapters 29

3.1 Chapter 1. Spatial capture-recapture toolbox for small 29 mammals

Spatial capture-recapture design and modelling for the study of small mammals

3.2 Chapter 2. Abundance indices validation

Using spatial capture-recapture modelling to calibrate indirect abundance estimates for the large-scale monitoring of a farmland rodent pest 
An assessment of capture biases that influence estimates of population sex-ratio in a fossorial rodent

\section{Discussion}

4.1 Brief summary of the main message of this thesis

4.2 The importance of detection probability for population monitoring

4.3 Capture-recapture studies and how they deal with detectability

4.4 Spatial capture-recapture studies

4.4.1 Making more use of SCR model outputs

4.4.2 Limitations of SCR models to our model species

4.5 Future research lines

5. Conclusions

6. Conclusiones

7. References

8. Acknowledgements

9. Appendix

9.1 Appendix 1

9.2 Appendix 2 


\section{ABSTRACT}

Gaining reliable estimates of population sizes is a fundamental aspect for many, if not most, ecological studies. The methods to do so, are continually adapting and as a result, these new methods must be tailored for particular groups or species to achieve these increasingly robust estimates of population sizes. Such methods, however, require substantial investments, both in time and money, to achieve fruition and alternative methods should be considered when they are sufficiently accurate depending on stakeholder requirements. As such, having rapid cost-effective surveying methods calibrated with the time-intensive methods is a crucial requirement in many applied systems. The common vole (Microtus arvalis) is a facultative agricultural pest that recently invaded farmlands in northwest Spain, causing crop damages and public health risks (e.g. transmission of tularemia to humans) during population outbreaks. There is thus, an urgent need to better understand how vole populations function to prevent and manage outbreaks. In this thesis, state-of-the-art models are applied to field data to fill important knowledge gaps related to field survey methods for common voles. Spatial capture-recapture (SCR) modelling offers a powerful tool to study elusive animals and precisely estimate population density and structure, and to study key basic ecological parameters. The thesis is based on the detailed study of a free-ranging common vole population in an experimental plot of 1.2 ha located in an agricultural landscape of NW Spain and on the use of capture-mark-recapture (CMR) methods applied every month over a two-year period during a density decline phase. I refined aspects of the CMR method and applied the SCR models to precisely estimate density and population structure (i.e. sex-ratio), and to obtain sex-specific insights on animal 
movement's space use or behavior. SCR models resolve many of the issues faced by classic capture-recapture (CR) methods, which do not consider the spatial structure of the ecological processes. This thesis consists of three main chapters: (i) the design of a toolbox that allows the application of SCR modelling for the study of small mammals, with a case study on common voles; (ii) a validation and calibrating of an indirect method of estimating vole abundance based on vole activity signs, commonly used for the large-scale monitoring of this farmland rodent pest, and (iii) an assessment of capture biases that may influence estimates of population sex-ratio in the common vole. After testing and adjusting the trapping methodology to the model species, the highlight results were: (i) a 5-fold reduction in trap mortality through the inclusion of a nest box coupled to the trapping device, (ii) the optimization of traps configuration (minimum distance between traps) to accommodate the movement of individual that were more sedentary (females), (iii) a seasonal variation in the detectability of vole activity signs associated with increasing vegetation height and reduced vole activity, (iv) a validation of the use of vole activity signs measured inside the field to predict vole densities up to 100 voles per ha, though with a poorer performance of vole signs measured in field margins, (v) an assessment of bias in adult sex-ratio estimated using capture methods, which are affected by season, sexual differences in baseline detection probability and movement. The results of this thesis allow the improvement of current methods used to estimate common vole density and will enable the study further key ecological parameters in the future. They have also provided a better understanding of the heterogeneities in capturability or detectability and potential bias associated with survey methods, particularly crucial given the 
study species' role as both pest and disease vector. Cumulatively, the outcomes of this thesis have the potential to help improve common vole monitoring, but similarly have a broader applicability to other similar species. 


\section{RESUMEN}

Obtener estimas precisas y fiables del tamaño de la población es un aspecto fundamental para muchos, si no la mayoría, de los estudios ecológicos. Los métodos para llevarlo a acabo se están adaptando continuamente y, como resultado, estos nuevos métodos deben adecuarse a grupos o especies particulares, con el fin de lograr estimas cada vez más sólidas del tamaño de la población. Tales métodos, sin embargo, requieren inversiones sustanciales, tanto de tiempo como económicas, para lograr dicho fin, por lo que deben considerarse métodos alternativos, cuando son lo suficientemente precisos, según los requisitos de las partes interesadas. Por lo tanto, contar con métodos de monitorización rápidos y rentables debidamente calibrados con los métodos que requieren mucho tiempo/dinero es un requisito crucial en muchos sistemas aplicados. El topillo campesino (Microtus arvalis) es una de las principales plagas facultativas de reciente invasión en tierras agrícolas en el noroeste de España, causando daños a los cultivos y problemas de salud pública (ej. transmisión de la tularemia a humanos) durante los brotes de población. Por lo tanto, existe una necesidad urgente de comprender mejor cómo funcionan las poblaciones de topillo campesinos para prevenir y gestionar estos brotes. En esta Tesis, se aplican los modelos estadísticos más avanzados hasta el momento y que, aplicados a los datos recogidos en campo, nos permiten mejorar los métodos para cuantificar topillos campesinos en su medio natural. Los modelos de captura-recaptura espacial (SCR, "spatial capture-recapture" en inglés) nos ofrecen una herramienta potente para poder estudiar animales con comportamientos difíciles de detectar, permitiéndonos obtener de una manera robusta, precisa e insesgada estimas de la densidad absoluta y de la 
estructura de la población, así como otros parámetros ecológicos clave. Esta Tesis se basa en el estudio detallado de una población silvestre de topillo campesino en una parcela experimental de 1,2 ha ubicada en un entorno agrícola del noroeste de España y en el uso de los métodos de capturamarcaje-recaptura (CMR) aplicados todos los meses a lo largo de un periodo de más de dos años durante una fase de disminución de la densidad poblacional. Se refinan los aspectos del método de CMR y se aplican los modelos SCR para medir con precisión la densidad y la estructura de la población (proporción de sexos) y obtener información específica sobre comportamiento y uso del espacio por parte de machos y hembras. Los modelos SCR resuelven muchos de los problemas a los que se enfrentan los métodos clásicos de captura-recaptura (CR), que no tienen en cuenta la estructura espacial de los procesos ecológicos. Esta Tesis consta de tres capítulos, cuyos objetivos son: i) el diseño de una serie de recomendaciones que permite la aplicación de los modelos de SCR para el estudio de pequeños mamíferos, con el topillo campesino como especie modelo, ii) validar y calibrar un método indirecto para estimar la abundancia de topillo basado en sus signos de actividad, comúnmente utilizado para el monitoreo a gran escala de esta plaga de roedores en tierras de cultivo, y iii) evaluar los potenciales sesgos en la captura que pueden influir en las estimaciones de la proporción de sexos en una población silvestre de topillo campesino. Tras probar y ajustar la metodología de captura en la especie modelo, los resultados más destacados son: (i) una reducción de 5 veces la mortalidad durante la captura mediante la adición de una caja nido acoplada al dispositivo de captura, (ii) la optimización de la malla de trampeo (distancia mínima entre las trampas) al movimiento de 
los individuos que se movieron menos (hembras), (iii) variación estacional en la detección de signos de actividad de topillo asociados al aumento de la altura de la vegetación y la reducción de la actividad en la superficie, (iv) la validación del método basado en el uso de signos de actividad de topillos para predecir densidades de hasta 100 topillos por hectárea medidos en el interior del campo y con un peor funcionamiento de los signos de actividad medidos en los márgenes de campo, y (v) una evaluación de los sesgos en la proporción de sexos adultos estimada utilizando diferentes métodos de captura, que son afectados por la estación, y las diferencias sexuales en la probabilidad de detección y el movimiento de los individuos. Los resultados de esta Tesis permiten la mejora de los métodos actuales utilizados para estimar la densidad de topillo campesino y permitirá el estudio de parámetros ecológicos clave en el futuro. También han proporcionado una mejor comprensión de las heterogeneidades en la probabilidad de captura o detectabilidad y el sesgo potencial asociado con otros métodos alternativos, especialmente destacado dado el papel de la especie de estudio como plaga y vector de enfermedades. Además de todo esto, los resultados de esta tesis tienen el gran potencial de ayudar a mejorar y optimizar la monitorización del topillo campesino, ofreciendo de manera similar una amplia aplicabilidad a otras especies similares. 


\section{Introduction}

\section{Foreword}

To understand how different biotic and abiotic factors influence the distribution and abundance of wildlife, it is necessary to measure quantitative changes of population size over time and space (e.g. population dynamics). Robust measurements of animal population abundances are the foundation for research, conservation and decision-making in wildlife management. Most researchers interested in the study and management of populations and their communities (e.g. ecologists, environmental managers, etc.) often formulate a basic question: "what is the best way to estimate the size of a wild animal population?" Yet, due to the cryptic nature, secretive behavior and heterogeneity in spatial distributions of many animal species, it is usually unfeasible to obtain a complete census or absolute count of individuals within a wild population. For this reason, researchers have to rely on the sampling of a proportion of the population and subsequently use statistical methods that take into account sources of variation in detectability (e.g., sex, time, habitat, etc.) to provide accurate and credible estimates of abundance or density of the whole population. Currently, there exists a great variety of methods to measure animal abundance, with different levels of precision and application limitations. The performance of each method (e.g. direct methods such as capture-markrecapture and catch-effort or indirect methods based on presence or abundance indices) will vary in relation to the ecology and behavior of the study species, the scale and design of the study, the habitat type, the time spent in the study and the logistics (e.g. human resources and material available). Using 
state-of-the-art of statistical methodology (i.e. spatial capture-recapture modeling) allows researchers to obtain reliable and accurate estimates of freeranging animal populations. A main aim of the present thesis is to consolidate, update and improve ecological knowledge (e.g. abundances, space use, sexratio) of a relevant species like the common vole. The common vole is keystone species for the functioning of many ecosystems (as an ecosystem engineer and prey for many predators), but when over-abundant common voles can have detrimental impacts on human health through their role as disease vectors, and also by becoming a pest for agriculture.

\subsection{Monitoring small mammal populations}

1.1.1 Obtaining reliable estimates and general applications of abundance variations

The main basis of population ecology studies revolves around population dynamics and studying which factors (intrinsic or extrinsic) determine or control the changes in the population size or abundance (e.g. studying the natural variations in abundance related to space and time or through experimental manipulation [Aplin et al., 2003]). Many factors have been suggested to cause population cycles, both extrinsic (e.g., weather, food supply, predation or disease) and intrinsic (e.g., social, dispersal, physiological and genetic factors) (Power, 1992; Sinclair \& Krebs, 2002; Korpimäki et al., 2004; Radchuk et al., 2016).

In order to properly monitor and explore various factors affecting populations (e.g. Cornulier et al., 2013), we need reliable, precise estimates of abundance (Güthlin et al., 2014; Krebs, 2014). Abundance is a crucial 
ecological parameter, necessary to understand the population dynamics and essential for the ecology, conservation and management of animal populations (Seber, 1982; Engeman \& Whisson 2006; Conn et al., 2006). Despite the considerable work developing methods capable of reliably estimating abundance, there remain nuances and complications for researchers and managers. For instance, it is imperative that abundance estimates consider, among other assumptions, the inevitable bias resulting from those animals that are present in a population but are not detected by the sampling methods in use (Ketz et al., 2018; Sabino-Marques et al., 2018; Jiménez, 2017).

Depending on the study aims, the absolute or relative sizes of the studied population can be assessed and estimated. The absolute size estimates can be used to get the density, i.e. the number of individuals in an area or volume (Krebs, 1978, 2014). Determining the density of a population in a manner that is cheap and easy to carry out is a common goal in many studies focused on basic ecological questions.

When estimates of absolute population size or density are not needed, population trend indicators or relative abundance indices can be used to obtain some knowledge of the spatial or temporal variation in abundance. In many cases, relative abundances are obtained by measuring biological indices (e.g. activity signs such as droppings, number of burrows, tracks, etc.) or similar signs that can be used to infer the presence or absence of the individuals in a given sampled area. Abundance indices can subsequently be validated and calibrated by comparing them with absolute density estimates obtained from more complex methods, therefore obtaining the desired cheap and easy method to carry out population studies or sampling protocols (Krebs, 2014). 
While such data may be used to answer basic ecological questions, they may similarly allow the study of other aspects such as population structure (e.g. age or sex composition within population).

Ecologists can use time series of animal abundance to explore predatorprey relationships (Gilg et al., 2003; Mougeot et al., 2019), examine the relationships between habitat and species (Hoek et al., 2015; Rodríguez-Pastor et al., 2016), or to evaluate the effects of experimental treatments in the study populations (Krebs, 2013; Paz et al., 2013). In a more applied context, wildlife managers often use abundance estimates to: (i) determine extraction quotas, making more precise decisions and evaluating the effectiveness of management actions (Delibes-Mateos et al., 2008), (ii) formulate conservation plans for rare or threatened species (Garrote et al., 2011) based on their distributions or population trends (Jiménez, 2017), (iii) understand the epidemiology of relevant pathogens (Acevedo et al., 2007; Gortázar et al., 2006) and (iv) establish management practices for wildlife control and pest management (Conn et al., 2006). Therefore, the preciseness of monitoring the abundance within populations is mandatory for any scientifically-grounded management or conservation plan, in order to establish simultaneous comparisons between populations, and to evaluate population dynamics over long periods of time (Battersby \& Greenwood 2003; Valente et al., 2014).

1.1.2 The study of small mammals: elusive and cryptic species that require specific methodology for monitorization

Small mammals are a broad grouping which includes the orders Insectivora, Rodentia, Lagomorpha, Carnivora and Chiroptera (Hayward \& Phillipson, 1979). 
For the purpose of this thesis, the focus will be on rodents. Small mammals represent a significant food resource for many avian and mammalian predator species (Aschwanden et al., 2007). Estimating the abundance or population density is challenging for small rodents (Rees et al., 2011). They are often nocturnal, with elusive habits, dwelling underground or beneath dense vegetation cover, and have relatively small home ranges (Williams et al., 2002). In addition, the population densities of most small mammals, such as Arvicoline rodents like voles, lemmings or gerbils, are highly variable even within small spatial scales, often varying within tens of meters (Castañeda et al., 2018) or inhabiting irregular environments and, therefore, showing a spatial structuring similar to a meta-population (Sabino-Marques et al., 2018).

When making population estimates, one of the main difficulties that arise is due to differences in the detectability of individuals within a particular a population. Imperfect detection is the general rule and not the exception, and a common characteristic that all field studies share (Schmidt \& Pellet, 2010). For example, different traits that characterize species, or individuals within a species, such as life history, size, behavior or rarity, can strongly affect detection probabilities (Iknayan et al., 2014). Likewise, individual traits, such as sex, age or distance to the observer, can be important causes of imperfect detection (Dénes et al., 2015). Detectability also varies due to specific factors of the study, like search/catching effort, observer, weather, sampling method, time of day or month of the year (Dénes et al., 2015). For instance, detection will vary between sites due to factors that influence visual or auditory detection independently of the observer, and/or because of differences in the habitat structure (Alldredge et al., 2007). 
Among small rodents such as voles, many species are elusive and cryptic, most are burrowers (i.e. they excavate galleries and chambers, spending much time underground [Brügger et al., 2010]). Complicating matters further, small rodents will often remain inactive for long periods, staying hidden during the day while making location effectively impossible while conversely being difficult to locate at night when they are active (Hansson, 1979; Boonstra et al., 1992). Some species are particularly difficult to detect when their numbers are low, such as the Cabrera vole (Microtus cabrerae), which is a threatened species both in Portugal (SNPRCN, 1990) and in Spain (Palomo \& Gisbert, 2002). These characteristics generally make it impossible to directly count or census wild species, e.g. counting all individuals (Dénes et al., 2015) and as a result, researchers often rely on alternative methods that are considered proportional to the size of the population, like abundance indices or individuals detected per unit of sampling effort (Buckland et al., 2008).

Small rodents have a widespread distribution, being particularly abundant in agricultural landscapes globally (e.g. Jacob 2003; Tattersall \& Macdonald, 2003), and are often considered a key component of ecosystems (Gauthier et al., 2011; Krebs, 2011) in the role as herbivores (Cornulier et al., 2013; Fischer \& Schröder, 2014). Many of these small-sized rodents are important seed predators (Daedlow et al., 2014; Fischer \& Türke, 2016), providing biological control by consuming seeds of plants considered weeds (Daedlow et al., 2014; Fischer \& Türke, 2016), which affect the growth, recruitment and population density of other plants (Pearson et al., 2014). From a broad ecological perspective small burrowing rodents are a key component of the food web, generating ecosystem services such as the aeration of the inner 
parts of the ground, can act as plant seed dispersal, may be involved in soil fertilization and through creation of burrows provide shelter for many other species such other small mammals, reptiles or amphibians (Martin, 2003; Jacob et al., 2014; Zhang et al., 2003). Moreover, voles are important prey species for raptors (Baker \& Brooks, 1981; Paz et al., 2013) and other generalist or specialized mammals like weasels (Graham \& Lambin, 2002; Tapper, 1979; Mougeot et al., 2019). However, when over-abundant, voles cause damage to agriculture and forestry (O'Brien 1994; Pugh et al., 2003; Jacob \& Tkadlec, 2010) or contribute to the transmission of diseases to humans (Pugh et al., 2003; Pikula et al., 2002) and are considered as pest species.

Arvicoline rodents, and in particular voles, are one of the most studied group of wild mammals (Stenseth \& Ims, 1993; Krebs, 2013; Jacob et al., 2014). Among this group we can find two extremes: (i) species, such as the common vole (Microtus arvalis), whose populations greatly fluctuate in abundance and have a profound impact on the dynamics of the resources they feed on and their natural vertebrate predators (Stenseth \& Ims 1993; Ims \& Fuglei 2005), and (ii) species such as the Cabrera's vole (M. cabrerae) which are threatened, rare, have very low overall abundance and typically occupy small habitat patches irregularly distributed (Pita et al., 2007). In the latter case, when the low numbers of individuals in a population may be critical, it is important to consider that the detection probability of a species in a specific place may strongly depend on its abundance (McCarthy et al., 2013; Warren et al., 2013).

For all the above-mentioned reasons, an effective and appropriate monitoring method is often desirable, one which may be adapted to specific 
case studies, as different factors contribute to varying detectability (e.g. abundance indices for abundant-pest species vs. genetic sampling (e.g. droppings) for rare, threatened species). Both situations would require a common and robust statistical method that deal with the problem of detectability. Depending on the aims, monitoring may be based on: (i) a fast and cheap method or a trapping/extracting method that allows implementation at a large spatial scale, for instance such as the monitoring of a common species that can become pests during population outbreaks, or (ii) a method that does not put the focal species at risk, when dealing with rare ones (i.e. threatened or protected species).

\subsection{Different methods to monitor the abundance of rodent populations}

Various monitoring techniques have been developed for ecological study of small rodents. It is rarely, if ever, possible to conduct a complete census of rodent population due to budget, time or manpower constrains, or the difficulty to attain all the individuals; therefore, managers often rely on estimates of population abundance (e.g. Engeman et al., 2013; Grimm et al., 2014).

The selection or application of a method to estimate animal abundance must consider aspects such as the logistical and financial resources available, the ecology of the species under study, the periodicity of sampling, and the management issues that are to be addressed (Mayle et al., 1999; Pollock et al., 2002; Valente et al., 2014; Wilson \& Delahay, 2001). The methods used to estimate small rodent abundance can be broadly classified as direct or indirect. 
In the direct methods, there is contact (e.g. visual observation, trapping and manipulation) with the monitored animal (Focardi et al., 2002; Lyra-Jorge et al., 2008; Rodríguez-Pastor et al., 2016). With indirect methods the animals are not seen, but their presence or activity is evaluated based on signs of such as tracks or runs, groups of pellets/faeces, recent excavation of burrows or feeding remains (Lyra-Jorge et al., 2008; Valente et al., 2014; Virgós \& Casanovas, 1999; Jareño et al., 2014; Gervais, 2010). Below I briefly describe alternative methods used to estimate abundance in small rodents, from the most detailed to the easiest.

\subsubsection{Direct methods based on capture-mark-recapture (CMR)}

The capture-mark-recapture (CMR) method is commonly used to estimate population size (Otis et al., 1978; Seber, 1982; Pollock et al., 1990; Amstrup et al., 2005). Currently, capturing and marking individuals by different means (e.g. collars, toe clipping, transponders under the skin of small rodents, ear tags) is the most effective technique for the study of different aspects of the ecology of wildlife species which require detailed information of population size. Livetrapping is considered to be the best technique for monitoring populations of small mammals (Flowerdew et al., 2004; Wiewel et al., 2007) from which diverse information can be obtained from the captured individuals, including species, sex, weight, physiological and reproductive condition, among others.

The CMR method is considered the "golden standard" for obtaining information on wild populations, but also has a number of disadvantages. In relation to the capture of rodents, this method requires significant financial, personnel, time, effort, and material investments such as a large number of 
devices (e.g. >100 traps) that are normally required to capture a sufficient number of animals (e.g. Batzli et al., 1983; Henttonen et al., 1987; Krebs et al., 1995, 2014; Romairone et al., 2018). In addition, various consecutive trapping sessions are required to obtain complete capture histories of multiple individuals (with the requirement that these individuals have a permanent marking for identification, that is, the individual's marking is present and detectable for the full duration of the study; Fauteux et al., 2018). Constant and repeated efforts to capture and mark individuals can be costly and even incompatible with certain conservation strategies, especially for long-term monitoring (Pollock et al., 2002). It can also be difficult to capture enough individuals to obtain robust statistical inference (Lindberg, 2012), and capture and tagging can affect the behavior of individuals. For instance, the probability of capturing animals can often change after being trapped for the first time, as some individuals may be "trap-shy" (inclination to avoid the traps) or "trap-happy" (prone to re-enter the traps; Yip et al., 2000), which may bias the observed individual capture/recapture histories.

Variants to CMR can be used which do not require physical capture or manipulation of individuals (Royle et al., 2014; Greenwood \& Robinson 2006; Jareño et al., 2014; Fauteux et al., 2018). Non-invasive "trapping" methods are increasingly popular, including the use of camera trappings (Karanth \& Nichols, 1998; O'Connell et al., 2011), acoustic recording devices (Dawson \& Efford, 2009) and methods based on fecal or hair samples, DNA extraction and genotyping in order to identify individuals (Royle et al., 2014; Sabino-Marques et al., 2018). While these methods could be considered to be easier than traditional CMR, they still require a substantial time and expensive investment. 
As such, researchers and managers may still prefer to rely on further alternative methods that provide indices of relative abundance (Jareño et al., 2014; Gervais, 2010).

1.2.2 Direct method based on a single catch effort (Single Trapping Sessions; STS)

The simplest direct method to estimate abundance is to set a sufficient number of trapping devices for a given time and report the number of individuals captured per unit of catching effort (Skalski et al., 2005; Buckland et al., 2008). This system has been used in many studies of small mammals (e.g. rodents) in order to estimate relative abundance (e.g. Rodríguez-Pastor et al., 2016; Quéré et al., 2000; Krebs, 2013; Mougeot et al., 2019), or to compare abundance estimates obtained by other alternative direct or indirect methods (e.g. Jareño et al., 2014; Fauteux et al., 2018). For example, the number of captured individuals/100-night traps (i.e. 1 night-trap = 1 operational trap set for $24 \mathrm{~h}$ ) provides an index of the number of individuals present in the sampled area, and can be used to compare the relative density of the animals in an area at different times or to compare density in different areas, provided that the same method of capture is used in all circumstances (e.g. Rodríguez-Pastor et al., 2016).

This method also allows researchers to collect other descriptive variables of the captured individuals, such as a naïve estimate of the population sex-ratio (Skalski et al., 2005). As with CMR, information can also be gathered to provide insights on population characteristics (e.g. age structure) while also allowing measurable samples (i.e. blood, parasites) to be collected from the captured 
individuals (e.g. Rodríguez-Pastor et al., 2017, 2019). Knowledge of a population's sex- and age-ratio, which may differ between species/populations, are essential to understand the reproductive potential of those populations (Wobeser, 2007). For instance, during the outbreak of a disease, it is possible to estimate the specific number of individuals in the population based on the sex of the affected and/or dead individuals in relation to a simple count or capture. However, such sex-ratio or age-ratio estimates may be biased by differences in the probability of detection of different classes of individuals (heterogeneity, Otis et al., 1978). Such issues may be most pronounced in species where males are more conspicuous or mobile than females, or due to the differential expression of the disease in between sexes. It is therefore important to bear in mind that the unequal distribution of a sex is sometimes the rule in some animal populations (Wobeser, 2007) but not always (equal proportion 1:1; Fischer, 1930). This potential bias tends to be greater when a small proportion of the population is captured, because the probabilities of capture and the number of capture occasions are low (Hilborn et al., 1976). Both abundance estimation and the accurate evaluation of the proportion of sexes in a population, often requires the use of other alternative methods or a regular sampling program that takes into account the probabilities of capture/detection based on sex, spatial and temporal activity (Nichols \& Pollock 1983; Kikkawa, 1964; Yoccoz et al., 1993). Understanding these potential biases may allow to correct estimates obtained from simpler methods (Bryja et al., 2005). 


\subsubsection{Indirect method based on Vole Activity Signs (VAS)}

Estimation of abundance is often been based on the use of descriptive indices (Schwarz \& Seber, 1999; Wilson \& Delahay, 2001; O'Connell et al., 2011), with the main assumption that the index, scalar or ordinal, is directly proportional to the population density of the study species.

Unlike capture methods, indirect methods are based on presence indices, based on signs of activity, to estimate the abundance of a species (Gervais, 2010; Jareño et al., 2014; Massei et al., 2018). These methods do not require catching or seeing individuals, and are therefore less expensive (Thompson et al., 1998), quicker and easier to apply and, subsequently, allow work to be carried out at larger spatial scales (Giraudoux et al., 1995; Miñarro et al., 2012). There exist a great variety of field techniques to obtain indices of abundance, and the most appropriate technique must be chosen depending on the study species and habitat where the research will be carried out (Gompper et al., 2006). Commonly, indices of abundance are based on signs of animal activity such as tracks, runs, burrowing activity, pellet/feces count, fresh clipping or signs of herbivory, etc. (Güthlin et al., 2014).

Abundance indices based on vole activity signs (VAS), such as the presence of fresh droppings, recent excavation with active burrows, or stored cut fresh vegetation, can be a useful alternative to the capture and marking of animals (Gervais, 2010; Jareño et al., 2014). Multiple studies have highlighted the utility of indirect methods to estimate abundance (Delattre et al., 1999; Lambin et al., 2000; Wheeler 2008; Terraube et al., 2011; Jareño et al., 2014), although in some cases, these indirect methods proved to be inaccurate 
(Gervais, 2010). A main limitation is also that no information is usually provided on the population composition (e.g. age, sex structure), except when combined with genotyping of feces which allow for sex and individual identification (e.g. Sabino-Marques et al., 2018; Ferreira et al., 2018; Piggot \& Taylor, 2003). Another important limitation of using an abundance index is that they are often not well validated or calibrated. In such cases, VAS can only provide a relative abundance, and not absolute abundance that can be compared in different contexts (Anderson 2003; Krebs et al., 2012). In addition, these indirect methods are less accurate and often prone to errors or biases (Tellería, 2004; Witmer 2005; Güthlin et al., 2014). For example, observer effects or differences in persistence or detection of signs between stations or habitats or changing weather conditions (e.g. rain) can lead to biased estimates (Güthlin et al., 2014). Obtaining reliable estimates of abundance is often a methodological

challenge for researchers and managers, so there is a constant need to develop better methods or validate existing ones, so that they are easy to use and inexpensive, and have the desired level of accuracy to be useful for ecological studies and management (Krebs, 1999; Tellería, 2004; Witmer, 2005; Jareño et al., 2014).

\section{3 Statistical analysis of capture-recapture data}

\subsubsection{Non-spatial analysis of capture-recapture $(\mathrm{CR})$ data}

The most widely used method for estimating the abundance of small mammals is the classical capture-recapture (CR) model (Seber, 1986; Krebs et al., 2011; Borchers \& Efford, 2008). However, a set of assumptions must be considered to estimate the population size properly based on CMR techniques (Krebs, 2014). 
The probability of recapture is an essential component for the functioning of these models (Royle et al., 2014). Some variables that may affect this parameter can be incorporated, such as the behavior of a previously captured individual, which could increase or decrease the probability of being recaptured, or the type of bait used (when traps are baited) or the capture history of traps (in which captured individuals may leave residual odours).

All CR models use a proportion of the animals present in a given area, with an important assumption that each animal has the same probability of being captured $(p=1)$ though this assumption is often violated in natural populations (Krebs, 2014). The animals captured for the first time are returned to the study population, which is sampled again, and the proportion of recaptured marked animals is used to estimate the population size. In this case, numerous capture opportunities are required to achieve reliable population estimates (Chao \& Huggins, 2005). It is important to highlight that traditional CR models have two main limitations: (i) the effective sampling area is imprecise (animals trapped on the edge of the trapping grid have a home range not fully included in the trapping area), and, (ii) there is a heterogeneity in the capture probabilities, due to the location of the centers of activity of individuals (Chandler \& Royle, 2013; Royle et al., 2013; Royle et al., 2014; Morin et al., 2017). Classical capture-recapture (CR) model assume that an animal has the same probability of being captured in all traps, when in fact they typically move around an activity center (e.g. burrow, colony) and are more likely to be trapped near this place. 


\subsubsection{Spatial analysis of capture-recapture data}

In the last 15 years, a new class of statistical tools has emerged, which have led to improvements in estimates of abundance and other key population parameters. The spatial capture-recapture (SCR) models (Efford, 2004; Borchers \& Efford, 2008; Royle \& Young, 2008; Royle et al., 2014, 2017) have recently been developed to simultaneously consider the factors that may affect among-individual variations in capture probability as well as the spatial location of traps and captures, thereby obtaining more accurate estimates of density, space use and population structure (Efford, 2004; Royle et al., 2014, 2017). These models are gaining popularity because they can be adapted to different types of capture data, collected using a variety of monitoring methods other than direct capture, such as non-invasive methods (camera trapping of recognizable individuals; DNA genetic sampling of hair traps or scats and genotyping of individuals) (Royle et al., 2014; Sollmann et al., 2013).

SCR models are commonly used to estimate absolute densities (Chandler \& Royle, 2013; Kéry et al., 2011; Royle et al., 2011), but their scope in different applications is constantly expanding (Jiménez et al., 2017; Royle et al., 2017), in such a way that these methods can be adapted to the study of small rodents, such as voles. SCR modelling is currently considered the most robust methodology, in comparison with other non-spatial CR methods (Parmenter et al., 2003; Krebs et al., 2011; Gerber \& Parmenter, 2015; Romairone et al., 2018). Like the non-spatial CR models, SCR models estimate ecological parameters other than density and consider the imperfect detection of individuals: the heterogeneity in capture probability. In other words, animals may vary in their capture probabilities according to age, sex, social status or 
other factors (Pollock, 1982). In addition, the SCR models use the information contained in the spatial configuration of detections and non-detections, to produce spatially explicit abundance estimates (Royle et al., 2014, 2017). This provides not only better estimates but also allows the mapping of the variation in abundance within the trapping grid.

SCR modeling provides absolute densities with an associated error and allows obtaining precise sex-ratio estimates and other ecological parameters, such as the information on movement (sigma parameter, $\sigma$ ). Sigma describes how the probability of detection decreases as a function of the distance between the trapping device and the activity center of an individual (which under certain circumstances is directly correlated with an individual's home range). The $\sigma$ parameter can be estimated by class of individuals (for instance by sex). Estimates on a behavioral parameter, the probability of baseline detection $\left(\lambda_{0}\right)$, are similarly computed. The basal detection probability can vary among individual classes (e.g. sex), over time (i.e. over successive capture occasions) or according an individual's capture history (i.e. trap shy vs trap happy individuals). Importantly, the estimation of these parameters (or covariates) not only generates more precise density estimates (all these factors introduce potential biases), but also informs on individual behavior and population composition. For instance, considering the influence of sex on capture probabilities or sigma not only allows to better estimate the abundance of each sex (and of the overall population), but also allows to explore to what extent individual behavior or home ranges may differ between sexes. 


\subsection{A case study on common voles (Microtus arvalis)}

The common vole (Microtus arvalis) is one of the most abundant rodents in Europe and is a main facultative vertebrate agricultural pest that can cause significant crop damages during population outbreaks (Jacob \& Tkadlec, 2010). The common vole is also a host and amplifier of diseases that affect humans (Han et al., 2015; Luque-Larena et al., 2017, 2015; Rodríguez-Pastor et al., 2017). In recent decades, the common vole colonized the agricultural landscapes of Castilla-y-León (northwest Spain), invading about five million of hectares of agricultural land where population irruptions are now common (Luque-Larena et al., 2013). Since the invasion, regular population outbreaks have occurred (Luque-Larena et al., 2013) and local populations have been shown to have cyclic population dynamics with a period of approximately 3 years (Mougeot et al., 2019). During common vole outbreaks in NW Spain, there have been claims of significant damages to crops and economic losses in agricultural areas. Moreover, the common vole has been shown to act as an amplifier of zoonotic diseases that have important implications for public health, such as tularemia and bartonellosis (Han et al., 2015; Luque-Larena et al., 2017, 2015; Rodríguez-Pastor et al., 2017; Rodríguez-Pastor et al., 2019). For all these reasons, there is a great interest in monitoring the abundance of populations of common vole, for which reliable estimates are required.

SCR modeling is a powerful analytical tool used to estimate density and obtain information on the use of space and the behavior of elusive and/or animals difficult to detect, like the common voles. Despite its great application potential in medium-large size species of carnivores (Royle et al., 2014; 
Sollmann et al., 2011), SCR has rarely been applied to the study of small mammals that are ecologically key such as voles (Krebs et al., 2011; Ergon \& Gardner, 2014; Sutherland et al., 2014; Sabino-Marques et al., 2018). This thesis aims to resolve this knowledge gap by applying the most advanced statistical methodology to date to the study of the common voles. Although most studies using SCR modelling have been focused on obtaining accurate density estimates for future management and conservation plans of certain umbrella species (Sollmann et al., 2011; Gray \& Prum, 2012), only a few studies made use of the ecological parameters that can be derived from SCR (i.e. the aforementioned baseline probability of detection, $\left[g_{0}\right]$, or the movement parameter, $[\sigma])$. To the best of our knowledge, only one paper by Casula et al., (2018) used the movement parameter $(\sigma)$ to study whether the home ranges of different rodent species decreased or increased in relation to the density of the other rodents.

Regarding the model study species of this thesis, it should be noted that within the group of voles, the common vole has certain characteristics that make it the most adequate to study variations in the population structure (Bryja et al., 2005). The common vole shows "boom-bust" dynamics, or multi-annual population cycles with peak abundances every 2-5 years (Lambin et al., 2006; Tkadlec \& Stenseth, 2001; Mougeot et al., 2019). Density can increase rapidly from very low numbers to a number above two thousand individuals per hectare in peak years (Bryja et al., 2005). 


\section{Objectives}

Through the different chapters of this thesis I will explore:

1) How to adapt the SCR methodology for the study of small mammals such as voles and make the best possible use of the robust estimates obtained and of the parameters that can be derived by using this methodology (chapter 1). In order to achieve this, the first issue to be resolved is related to mortality associated with live-trapping, which can be high in small mammals and must be minimized given SCR requires recaptures. A nest box coupled to a classic Sherman trap was designed and tested in order to reduce vole mortality in traps. Second, the trapping grid design had to be adapted to the home range of individuals in order to maximize spatial recaptures. This first chapter aims at providing the basic methodology to successfully apply SCR modelling to a species like the common vole.

2) The performance of a commonly used Vole Activity Sign (VAS) index, an indirect method frequently used to monitor small rodent abundance frequently and at a large spatial scale (e.g. regional). The method used must be easy, simple and inexpensive to allow for large-scale spatial monitoring (Delattre et al., 1990; Jareño et al., 2014). As knowledge of the species studied increases and new methods become available, it is important to review and reexamine the reliability of the methods used to measure abundance (Embleton \& Petrovskaya, 2013; Lisická et al., 2007), to allow for more reliable monitoring, forecasting and decision making. The limitations of both time and budget make the CMR or SCR methods less suitable when large-scale continuous monitoring of small mammals is required. By contrast, indirect methods are more 
affordable (Thompson et al., 1998), quick and easy to implement and, therefore, allow working at large scales (Giraudoux et al., 1995; Miñarro et al., 2012). However, they are less accurate and prone to several sources of errors (Telleria, 2004; Witmer, 2005; Güthlin et al., 2014). Vole Activity Sign (VAS) method is currently being used by the regional government, ITACyL, as part of their regional monitoring of vole outbreaks in Castilla-y-León. The specific aims of this chapter (chapter 2) will be to validate and calibrate the VAS method by comparing it with the more precise method based on CMR and SCR. Using two years of consecutive monthly sampling with both methods, comparison will be made using the estimates obtained using two alternative methods and explore how to best use the VAS to predict vole abundance.

3) Population sex-ratio variation in adult common voles, and the potential biases in estimating population sex-ratio using single capture data. Because capture probability can greatly vary among individuals, and in particular between sexes, the estimates of population sex-ratio derived from single trapping sessions can be biased. In chapter 3, sex-ratio estimates obtained using Single Trapping Sessions (STSs) are compared with those obtained using CMR and SCR modeling, in order to study and correct the biases inherent to the former method (owing to potential differences in capturability of male and female voles). Obtaining reliable estimates of the abundance of males and females within a wild population, and therefore the sex-ratio, can be a challenge for the study of small mammals due to their elusive and cryptic lifestyle. This is because abundance estimates are derived directly from different capture methods and, therefore, are sensitive to potential sexual differences in in trapping rates (Bryja et al., 2005). Both sexes can profoundly differ in their 
behavior, movement and ecology (for example, male mate searching behavior during the breeding season). The heterogeneity in the probability of capture between males and females can affect not only the estimates of population abundance, but also the estimates of the sex-ratio (McKnight \& Ligon, 2017). Hence the importance of calibrating these results with robust estimates obtained from methodologies that do take into account factors such as the heterogeneity in the probability of captures of individuals in the population. 


\section{Chapters}

\subsection{Chapter "1"}

SCR toolbox for small mammals

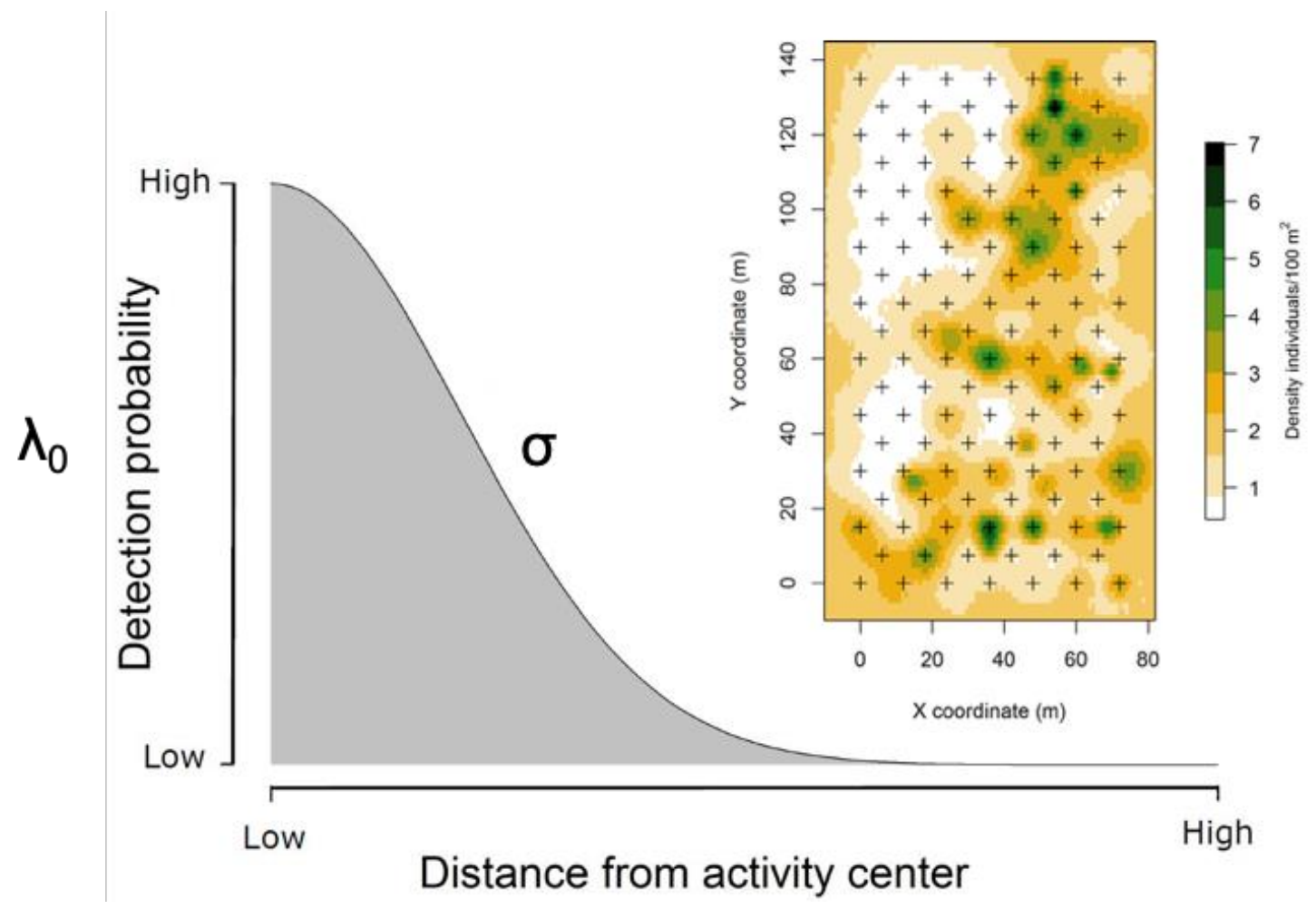




\section{Spatial capture-recapture design and modelling for the study of small mammals}

\section{Abstract}

Spatial capture-recapture modelling (SCR) is a powerful statistical method to derive both density and information on space use and behavior of elusive animals. Yet, SCR has seldom been applied to small mammal species which are central within their ecosystems (i.e. ecologically keystone). Within this chapter I show both the potential and requirements of SCR, using common voles (Microtus arvalis) as a case study species. First, I sought to minimize mortality associated with capture in live-traps, often high in small mammals, and must therefore be kept minimal. A nest box coupled with a classic Sherman trap was designed and led to a 5 -fold reduction of mortality in traps. Second, ensuring that the trapping grid is suitably arranged according to any given individuals home range, thus maximizing spatial recaptures. In May-June 2016, 227 voles in a 1.2-ha area during two monthly sessions were captured and tagged with transponders. Using a Bayesian SCR with a multinomial approach, the following were estimated: (1) the baseline detection rate and its associated variation according to sex, time or behavior (aversion/attraction after a previous capture); (2) how detection probability declines as a function of the distance to an individual's activity center $(\sigma)$, and investigated $\sigma$ variation according to sex; and (3) density and population sex-ratio. I show that reducing the maximum distance between traps from 12 to $9.6 \mathrm{~m}$ doubled spatial recaptures and improved model predictions. Baseline detection rate increased over time (after 
overcoming a likely aversion to entering new odorless traps) and was greater for females than males in June. Males appeared to have larger home ranges, indicated by the $\sigma$ parameter of males being twice that of females. Vole density estimates were $142.92 \pm 38.50$ and $168.25 \pm 15.79$ voles/ha in May and June, respectively, with 2-3 times more females than males. I highlight the potential and broad applicability that SCR offers and provide specific recommendations for using it to study small mammals like voles.

Keywords: mortality, trapping grid, spatial recaptures, movement, home range, density, sex-ratio, common vole, Microtus arvalis 


\section{Introduction}

A challenging yet crucial task for ecological studies is to obtain accurate estimates of population size or density, to inform both conservation and managementand species. The challenge is increased further when working with species that are cryptic or elusive. With such species, the traditional approach has been to use capture-recapture data obtained from live-trapping sessions. The most widely method used for estimating the abundance of small mammals is classic capture-recapture (CR) modelling (Seber, 1986; Krebs et al., 2011; Borchers \& Efford, 2008) and is referred to as the "golden standard" compared to indirect methods that estimate abundance based on activity signs (Jareño et al., 2014). Briefly, CR modelling uses a proportion of the real number of animals in a given area and assumes that each animal has an equal probability of being captured. Animals captured during the first encounter are released back to the population, which is then resampled, and the proportion of tagged animals is used to estimate the population size. In this case, numerous capture occasions are required to achieve reliable population estimates (Chao \& Huggins, 2005).

Despite the widespread use of Classic CR models, they have two apparent limitations. The first the effective sampling area is imprecise, and second, there is heterogeneity in capture probabilities between traps, due to the locations of individuals' activity centers (Chandler \& Royle, 2013; Royle et al., 2013; Royle et al., 2014; Morin et al., 2017). Advances to the classic CR models, such as spatial capture-recapture (SCR) models, have been developed to resolve these two issues. Namely, SCR makes inferences about population density from capture-recapture data in combination with spatial information from 
the locations of captures and traps (Borchers \& Efford, 2008; Efford, 2004; Royle \& Young, 2008; Royle et al., 2017). These models have shown great potential and are increasingly widely used for studying large or medium size mammals such as elusive carnivores (Royle et al., 2014; Sun et al., 2017; Sollmann et al., 2011; Gardner et al., 2010; Sollmann et al., 2012; Royle et al., 2009). Yet, despite their great potential, SCR models have so far seen little application in small mammal studies, such as voles (Krebs et al., 2011; Ergon \& Gardner, 2014; Sutherland et al., 2014). Many vole species are key to the functioning of their ecosystems, while also often being problematic when they reach high densities (becoming pests and leading to human wildlife conflicts) and constitute one of the most well-studied mammal groups (Stenseth \& Ims, 1993; Krebs, 2013; Jacob et al., 2014). Improving population density estimate precision is, therefore, paramount to provide reliable estimates of vole numbers and their dynamics.

All capture-recapture models (CR and SCR) have technical limitations that need to be addressed when setting up a study, as these will influence the quality of the data and subsequently model performance. Such limitations include trap-induced mortality, which can be very high in small mammals (Stephens \& Anderson, 2014; Montgomery, 1980; Eccard \& Klemme, 2013), and trap saturation when using single-catch devices (Krebs et al., 2011; Distiller \& Borchers, 2015; Taylor et al., 2011). In addition, SCR models require a specific spatial organization of traps in the study area that depends on individual movements (Efford, 2004; Sollmann et al., 2012; Sun et al., 2014). I show here how these three technical limitations were overcome in order and 
how these may lead to recommendations for others when studying vole populations.

Trapping animals will often result in the death of some captured individuals (especially when trapping small mammals). Therefore, when individuals to be live-trapped, marked and recaptured, introducing additional sources of mortality can seriously compromise the quality of the data (Jung \& O'Donovan, 2005) and bias the results of capture-mark-recapture studies (Rosenberg \& Antony, 1993). The risk of mortality in traps can be high for small mammals, notably due to hypothermia, dehydration, stress or food shortage (Montgomery, 1980; Stromgren, 2008; Stromgren \& Sullivan, 2014). For instance, in a trapping study in Oregon (USA), small mammal mortality in traps varied dramatically from $6.8 \%$ to $64.3 \%$ depending on species (Dizney et al., 2008). Another study in Wisconsin (USA) showed mortality rates in Sherman traps ranged from $<5 \%$ in deer mice (Peromyscus spp.) to c. $10 \%$ in Arvicolinae rodents and $>75 \%$ in shrews (Stephens \& Anderson, 2014). In western France, mortality of common voles (Microtus arvalis) in traps throughout a capturemark-recapture study averaged 11\% (Pinot et al., 2016). Such risks can be mitigated by using devices added to traps, such as a wooden end-chamber filled with cotton (Noguerales et al., 2015), a nest box (Rosenberg \& Anthony, 1993) or plastic box covering the trap (Klemola et al., 2002) that can enhance the survival of captured individuals.

Trap saturation, which is when there is a high proportion of traps occupied meaning there is limited to no possibility for new captures (Distiller \& Borchers, 2015), and is an additional metholodogical limitation, particularly when population density is high. When trap saturation occurs, this may induce a 
negative bias in density (D) estimates (Royle \& Converse, 2014; Efford et al., 2009). Trap saturation can be avoided simply by adding more traps (Royle \& Converse, 2014) or increasing the frequency of trap checks, so that the new captures further improve the accuracy of the estimates (Thomson et al., 2009).

Of particular importance in SCR are the home range size of the target species, and therefore the spatial organization of traps and the maximum distances between traps (Sun et al., 2017; Sollmann et al., 2012; Sun et al., 2014), which determine the resolution of the information obtained on individual movement (Parmenter et al., 2003; Wilson \& Anderson, 1985). For instance, if the distance between traps is too large, no information on animal movements may be inferred, because a given animal will only be captured in one trap (Dillon \& Kelly, 2007).

Each species may display additional and particular complexities for the estimation of model parameters, which can be included in the modelling process. Most vole species are difficult to detect. Low detection probabilities arise because of their burrowing life style -they spend a variable amount of their time underground (Brügger et al., 2010) or because of specific foraging tactics (Jacob \& Brown, 2000; Verdolin, 2006). Modelling allows to investigate the influence of different factors on capture probability (Willson et al., 2011; Otis et al., 1978) such as differences between sexes or classes of individuals (Ylönen et al., 1990), differences in behavior (degree of trap-happiness, i.e., willingness to re-enter a trap (Tanaka, 1963) or any influence from the trapping devices; neophobia; influence of residual odors from previous captures (Brouard et al., 2015; Krebs \& Boonstra, 1984). These model considerations will provide useful 
information about the study species and population, and, at the same time, reduce uncertainty around density estimates.

In the present study, I report on specific 1) technical considerations such as: mortality in traps, trap saturation, and spatial organizations of traps, and 2) modelling considerations that take into account variation in detection probability (i.e. sex, time or behavior) or movement. All these considerations should be taken into account for successfully applying different models for the study of small mammals in general. I provide and test the effectiveness of a technical improvement to reduce mortality during trapping (the addition of a nest box to single-capture traps) and consider the influence of using new versus previously used traps, the number and spatial organization of traps (trap spacing relative to individual movement) to minimize trap saturation and maximize spatial recaptures. SCR allows to obtain accurate estimates of population density (with an associated error) and other relevant structural and functional parameters in the studied population, such as sex-ratio, space use and movement. I illustrate this using an empirical study of a free-ranging population of common voles (M. arvalis) from an experimental field located in NW Spain. 


\section{Material and methods}

\subsection{Study species and area}

The common vole is a small sized herbivore $(<100 \mathrm{~g})$ distributed from Northern Spain to the Middle East and Central Russia; it is one of the most abundant rodents in Europe and a facultative agricultural pest (Jacob \& Tkadlec, 2010). Some common vole populations are truly cyclical (Tkadlec \& Stenseth, 2001), while other populations seem to fluctuate irregularly (Jacob \& Tkadlec, 2010). In northern and central Europe, multiannual population outbreaks are a common feature and occur regularly every 2-5 years (Cornulier et al., 2013). Some common vole populations cause important crop damages and economic loss during outbreaks in farmland areas (Jacob \& Tkadlec, 2010). In addition, they act as reservoir and amplifier of zoonotic diseases that have important public health implications (Luque-Larena et al., 2015; Rodríguez-Pastor et al., 2017; Luque-Larena et al., 2017; Han et al., 2015). The study was carried out in a large intensive agricultural region of NW Spain (northern plateau, "Tierra de Campos", Castilla-y-León region) where the farming landscape consisted of a mosaic of crops dominated by non-irrigated cereals (Jareño et al., 2015).

2.2. Nest box design and its influence on small mammal survival

In order to increase the survival rate of captured small mammals, I developed a nest box that was coupled to a classic Sherman trap $(8 \times 9 \times 23 \mathrm{~cm}$; LFTA Sherman). I created a device that has some operational similarities with the Longworth trap (Longworth Scientific Instrument Co., Oxford, England), which consists of a nesting chamber box coupled to a tunnel with a trigger and trap 
(Chitty \& Kempson, 1949). The Longworth trap is made of metal (usually aluminum), which provides poor insulation from cold or heat for the trapped animal. In a comparative study between live-trapping devices, Longworth traps had a reduced capture efficiency compared with Sherman traps in some environments (Anthony et al., 2005). Another study (Jung, 2016), comparing Longworth, Sherman and Uggland traps (Granhab, Gnosjö, Sweden), found a slight difference in capture efficiency between the three trapping devices, which was lower in Sherman traps and similar in Longworth vs. Uggland (Jung, 2016; Lambin \& MacKinnon, 1997). Here, I used the Sherman trap, which is made of metal, foldable and has an efficient trapping mechanism, but I added to it a nest box made of thick plastic material to provide an additional shelter with better insulation for trapped animals. Each nest was made using a section of a P.V.C drainage plumbing tube (diameter of $125 \mathrm{~mm}$, length of $150 \mathrm{~mm}$ and thickness of $3.75 \mathrm{~mm}$ ). Both sides of the P.V.C tube were sealed with end caps, and in one cap a hole was cut following the shape and dimensions of the shortest side of a Sherman trap, allowing its coupling with the nest (Appendix 1). The back door (i.e., the one located after the trap trigger) was removed to allow free movement of caught animals into the nest, while the front (trigger) door remained operational to prevent escape. During trapping sessions, nests were provided with dry bedding material (paper stripes) and baited with carrot, apple and rodent pellets (Global Diet 2018, Teklad).

I tested the effectiveness of the nest box at improving the survival of captured small mammals during trapping sessions of a seasonal monitoring program conducted in Tierra de Campos (Jareño et al., 2015; Rodríguez-Pastor et al., 2016). This monitoring consisted of regular vole trappings every 4 months 
(in March, July and November) in fields of cereal, alfalfa or in fallows. During the capture sessions of November 2016, March 2017 and July 2017, I sampled 60 field margins (20 each month), a linear habitat feature that hosts a large proportion of the vole population (Rodríguez-Pastor et al., 2016). I set up 10 traps in each field margin spaced every 2-m and alternated traps with and without a nest (5 in each group; see Appendix 1). Traps were set in the morning and checked and retrieved after $24 \mathrm{~h}$. For each captured mammal, I recorded the species, if it was alive or dead and if the trap had a nest or not. I used these trapping data to compare the proportion of each small mammal species that was retrieved alive from traps with or without nest. Most captures $(n=430)$ were of common vole (66\%), but I also captured wood mouse (Apodemus sylvaticus) (14\%), the Algerian mouse (Mus spretus) (16\%) and common weasel (Mustela nivalis) (4\%). I also report on the effect of nests on the survival of these other species in traps.

\subsection{Experimental field and spatial capture-recapture study}

The SCR study was conducted in May and June 2016 in an experimental field of 1.2 hectares managed by the Universidad de Valladolid. The experimental field is located in Soto de Cerrato ( $\left.41^{\circ} 94^{\prime} \mathrm{N}, 4^{\circ} 42^{\prime} \mathrm{W}\right)$, Castilla-y-León region. The area is characterized by an intensive agricultural landscape with cereal, alfalfa, and fallows (Jareño et al., 2015; Rodríguez-Pastor et al., 2016). The experimental field was planted with alfalfa ("Aragón" variety, non-irrigated) in spring 2015, and enclosed by a wire mesh fence of $2.5 \mathrm{~m}$ height to prevent access from people while allowing small mammals to move through it. 
Common vole monitoring within the enclosure was carried out using a capture-mark-recapture method in a marked study grid. All trapping devices were classic Sherman traps fitted with a nest (as described above). Each trap was also covered by a large U-shape concrete block to protect it from heavy rain, direct sunlight and frost (Appendix 1).

In May 2016, I set up a trapping grid consisting of 140 traps placed in pairs at 70 grid nodes regularly spaced $\left(72 \times 135 \mathrm{~m}, 9720 \mathrm{~m}^{2}\right.$ area) within the experimental field, with a minimum distance between traps of 12-m (Figure 1A). I set up two traps at each node in order to avoid trap saturation, which could result in poor model estimates (Distiller \& Borchers, 2015). I favored singlecapture traps because they were found to have a better trappability than multicapture traps in arid environments (Ylönen et al., 2003) and Nearctic temperate regions (Jung, 2016). In June 2016, the trapping grid was modified in order to reduce the minimum distance between traps and investigate effects on spatial recapture rate (see Results). The altered grid then consisted of 124 traps regularly spaced (Figure $1 \mathrm{~B}$ ) with one trap per node and a minimum distance between traps of 9.6-m. Preliminary analysis of the May data indicated that trap saturation was low and not an issue, but the information on vole movement indicated that the minimum distance between traps should be reduced (Figure 1B). 

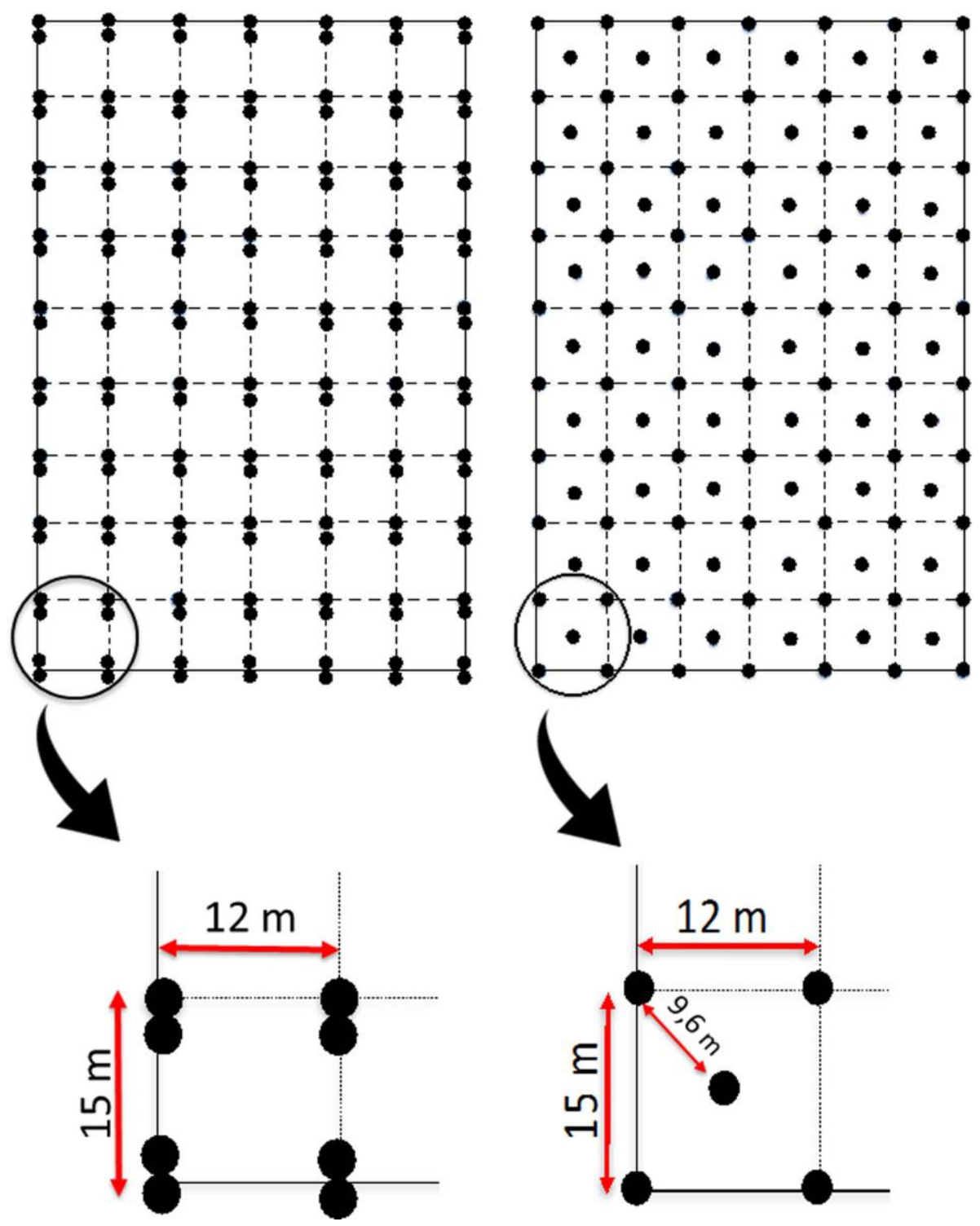

Figure 1. Spatial organization of the traps (grid design) used for the SCR study. In May (A) the trapping grid included 140 traps (black dots), set up in pairs at 70 points spaced by 12 or $15 \mathrm{~m}$. In June (B) the grid included 124 traps set up singly at 124 points, with distances between traps of 15, 12 and $9.6 \mathrm{~m}$. The overall trapping array covered 9720 $\mathrm{m}^{2}(72 \times 135 \mathrm{~m})$.

Capture sessions consisted of two sampling periods (May and June) separated by a 2-weeks interval without trapping (Appendix 1). Each period was organized in 4 consecutive days of trapping, 3 days without trapping, and another 4 consecutive days of trappings with a total of 8 capture occasions 
during each session. Traps were checked twice a day (9:00 a.m. and 4:00 p.m.). Each captured vole was individually marked with a transponder (Glass tag, BIOGLASS 8625, 2x12 mm, Ref: ICAR 941), sexed, weighed with an electronic balance to the nearest $0.1 \mathrm{~g}$, tail length measured with a ruler to the nearest $1 \mathrm{~mm}$, and then released at where it was captured. I also kept the right ear tip of each tagged vole as a source of DNA for future studies. This also allowed me to confirm that none of recaptured voles with a cut ear had lost its transponder or could not be identified because of transponder failure.

\subsection{Statistical analyses}

\subsubsection{Nests and small mammal survival in traps}

I tested for each species separately whether the presence or absence of a nest affected the probability of a captured small mammal to die in trap. For common voles, the only species for which I had a large sample size $(n=284)$, I modelled survival probability ( 1 = survived, $0=$ died in trap) using a binomial mixed model that included the field margin identity as a random factor (to account for the non-independence of data from the same field margin), the factor Nest (presence $=1$ versus absence $=0$ of a nest), the Month and the interaction Nest $\times$ Month, to test if the influence of the nest on survival differed between months.

For all the other species, the limited sample size prevented me from implementing mixed-models that included the field margin identity as a random factor (that failed to converge). I thus report results of simpler binomial models (as for the common vole, but without random effect), in order to report the effect of nest on survival. These models without random effect included Month, and 
thereby broadly controlled for differences in seasonal weather conditions during trapping but did not allow to control for variations in microclimatic conditions, at field margin level.

2.4.2. Model considerations and parameters/covariates evaluation

In order to consider sex differences in movement and home range (Royle et al., 2014), I tested models in which the movement parameter sigma $\sigma$ (the rate at which detection probability declines as a function of distance) differed between sexes $\left(M \sigma_{\text {sex }}\right)$. I also analyzed models where the baseline detection rates (the probability of detecting an individual at its activity center), $\lambda_{0}$, can vary depending on three covariates: 1) sex $\left.\left(M_{\text {sex }}\right), 2\right)$ behavior $\left(M_{b}\right)$, and 3) time $\left(M_{t}\right)$. The time effect considered that $\lambda_{0}$ varies among capture occasions, for instance due to a progressive adaptation of voles to the presence of traps over time. This is especially relevant given that small mammals may behave differently towards "new" than towards "previously used" traps. The behavioral effect considered the degree of boldness of voles towards traps after their first capture (Réale et al., 2007). After first capture individuals may become "trap shy" (less likely to enter a trap) or "trap happy" (more likely to enter a trap), or indifferent (Gerber \& Parmenter, 2015). Finally, one sex may be more detectable than other (because of differences in home range, activity rhythms, or other traits), and this must be taken into account to improve parameter estimates and obtain more precise estimations of density and population sex-ratio (Bryja et al., 2005). Interactions between covariates (time, sex and behavior) were not included in initial models because of sample size limitations (low number of number of recaptures; see Results). 


\subsubsection{Spatial capture-recapture modelling}

I estimated density and abundance within the state space $S$, that is an explicit spatial region within which the sampling of individuals occurs. It includes the trapping grid (spatial array of traps), and a buffer to ensures sufficiently large to include all individuals potentially exposed to sampling. In order to develop the state space in SCR, I used the values of $\sigma$ obtained from a preliminary analysis, and the buffer was created using a distance at least 2.5 times the $\sigma$ parameter of the half-normal from SCR model, beyond which an individual external from the trapping area would not be detectable. In the case study, I used a 30-m buffer amplification around the trapping grid.

Our capture devices are single-catch traps used during 8 capture occasions each month. Multi-catch or independent multinomial distributions are used when the capture probability of an individual in a trap is not independent of its capture probability in other traps, and when the capture of a given individual does not affect the capture of other individuals. This last condition may sometimes be violated when using single-catch traps, but a simulation study (Efford et al., 2009) showed that the multi-catch model is still adequate for single-catch traps when saturation is below $86 \%$ (which was the case in our study). To date, no likelihood function currently exists for single-catch traps (Efford et al., 2009) (but see (Distiller \& Borchers, 2015)). I therefore fitted a multinomial model using a Bayesian approach, accounting for dead individuals (Royle et al., 2014; Jiménez et al., 2017) and carrying out model selection on the various models described above. I took into account mortality in traps by adding a binary matrix that indicated during which capture occasion an individual died, so that it was no longer considered afterwards. I favored a 
Bayesian approach because in BUGS (Spiegelhalter et al., 2003) the construction of even very complex models becomes relatively feasible, transparent and easy to understand (Kéry \& Schaub, 2012). The Deviance Information Criterion (DIC) is not appropriate for model selection with mixed models such as SCR models (Hooten \& Hobbs, 2015). Here, I first evaluated if the $\sigma$ parameter differed between sexes. Then, using the selected model, I fitted all covariates that could potentially affect vole detection (time, behavior and sex) using the logit function:

$$
\operatorname{logit}\left(p_{0}[i, k]\right)=\alpha_{0}+w[1] \cdot \alpha_{1} \cdot \operatorname{time}[k]+w[2] \cdot \alpha_{2} \cdot C[i, k]+w[3] \cdot \alpha_{3} \cdot \operatorname{sex}[i]
$$

Where I are the individuals; $\mathrm{k}$ the occasions, and $\mathrm{C}$ a binary matrix that was used to account for differential behavioural responses of individuals to survey devices related to different capture histories, where $\mathrm{Ci}, \mathrm{K}=1$ if individual I was captured at least once prior to session $\mathrm{k}$, otherwise $\mathrm{Ci}, \mathrm{K}=0$. I did not included interactions between covariates due to sample size limitations (number of recaptures and spatial recaptures; see Results). For model selection, I used the Kuo \& Mallick (1998) indicator variable (w) selection approach to select the best candidate model in relation to the use of both parameters in the models (Royle et al., 2014) and I evaluated the sensitivity of posterior model probabilities to different prior specifications using a normal distribution $\mathrm{N}\left(0, \sigma^{2}\right)$, with $\sigma^{2}=10$ and $\sigma^{2}=100$. All these SCR models can also be fitted in a Maximum likelihood estimation framework using secr (Efford, 2016) or oSCR (Sutherland et al., 2018), with the advantage of using the Akaike Information Criterion (Akaike, 1974) for model selection. I have also done this alternative model selection (see Appendix 1). 
Models were run in Nimble (de Valpine et al., 2017). I ran 3 chains of the Markov Monte Carlo (MCMC) sampler with at least 150,000 iterations in each case (see Appendix 1). To check for chain convergence, I assessed MCMC convergence by visually inspecting trace plots for each monitored parameter, and I calculated the Gelman-Rubin statistic using the R package coda (Plummer et al., 2006) where values below 1.1 indicated convergence. For all parameters in our models, $R$-hat was always $<1.1$. Details of the models (scripts and outputs) are given as Supplementary Information (Appendix 1).

\subsubsection{Testing and simulations}

In order to better understand the results and check for possible sources of error, I also used our data to 1) evaluate trap saturation levels at the scale of an average common vole home range and 2) to simulate the influence of the trapping grid configuration on key parameter estimates (abundance and $\sigma$ ).

To estimate saturation at the scale of common vole home range, I considered an average circular area of maximum detection of $2.5^{*} \sigma$ radius (Royle et al., 2014) and used our trapping data in June to calculate for each trap and occasion the proportion of neighboring traps not available for capture within $a<2.5^{\star} \sigma$ distance around each trap (Appendix 1).

For simulations, I randomly generated the locations of individual voles using the June model outputs (population density and $\sigma$ ) using a script modified from (Royle et al., 2014) and either the trap configuration used in May or the one used in June (Appendix 1). I did four separate simulations (for each sex and trap configuration) using the Nimble software (de Valpine et al., 2017), with 
100 simulated populations in each case, 5000 iterations and a 1000 burn-in. I determined the error in the estimated population size and $\sigma$, calculating the root mean square error (RMSE) of these study parameters for each sex and trap configuration. 


\section{Results}

3.1. Effects of nests on small mammal survival in traps

I found a strong effect of adding a nest box on the survival of common voles during trapping, which differed between months (Mixed model; significant Month $x$ Nest interaction: $x^{2}=6.10 ;$ d.f. $\left.=2, P=0.047\right)$. In July, survival probability was highest and did not differ between traps with or without nest $\left(X^{2}=1.32\right.$, d.f. $=1$, $P=0.25 ;$ Figure $2 A)$. In March, survival probability was significantly higher in traps with nests than in those without nests $\left(X^{2}=6.82\right.$, d.f. $=1, P=0.009$; Figure 2A). In November, survival probability was overall lowest, and was also significantly higher in traps with nests than in traps without nests $\left(X^{2}=54.90\right.$, d.f. $=1, \mathrm{P}<0.001 ;$ Figure 2A) 

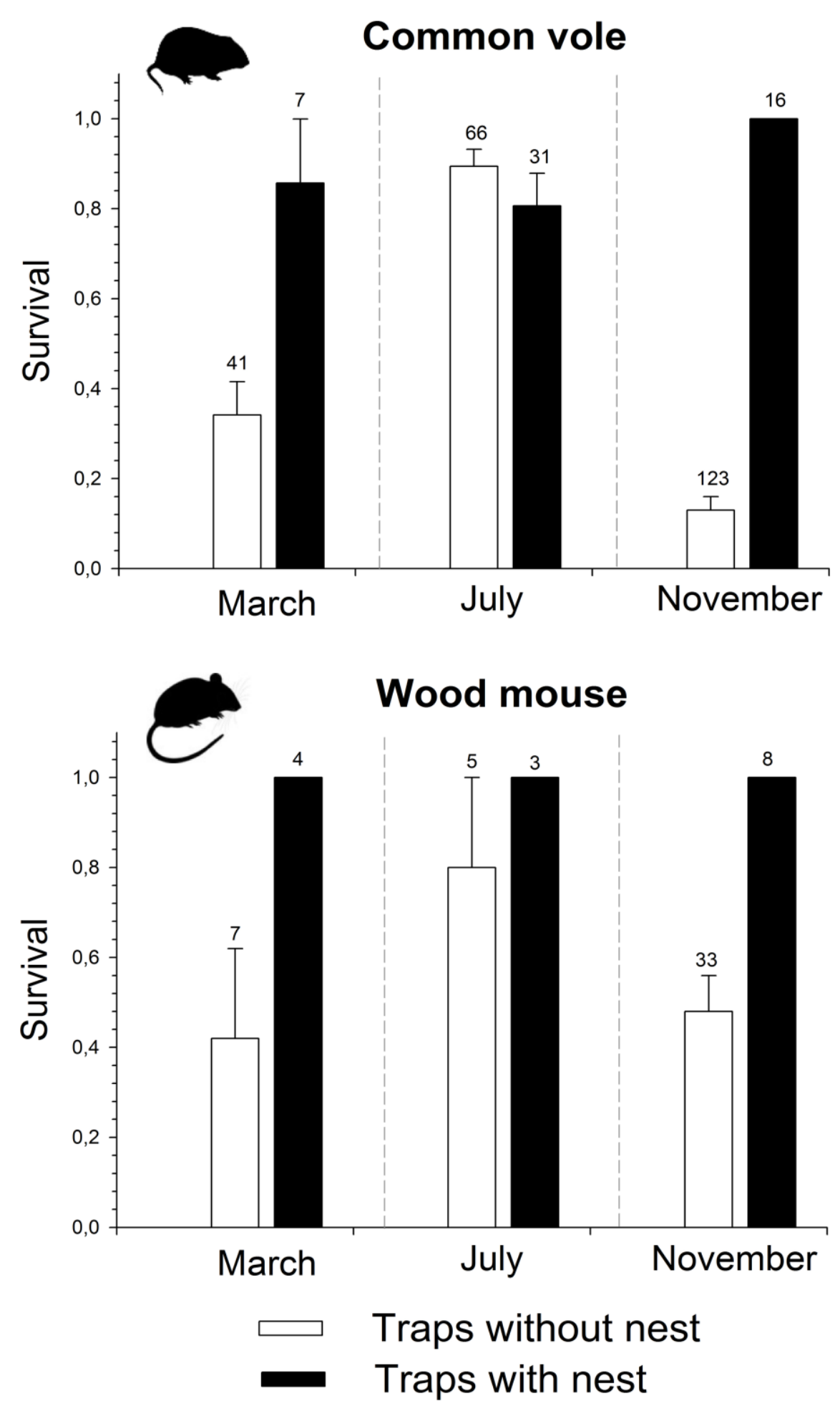

Figure 2. Effect of the addition of a P.V.C nest box to a trap on the mean ( \pm SE) survival probability of captured common voles (Microtus arvalis; upper) and wood mouse (Apodemus sylvaticus; bottom) according to month. Traps were set for $24 \mathrm{~h}$, alternating traps without nest (white bars) or with nest (black bars) in the same field margins. Number above error bars refer to the number of captured individuals. 
In the wood mouse, I found that survival probability was significantly higher in traps with than without a nest $\left(X^{2}=15.80\right.$, d.f. $=1, P<0.001$; Figure 2B), with no significant differences between months $\left(X^{2}=2.08\right.$, d.f. $=2, P=$ 0.354), and no significant interaction Month $x$ Nest (Figure 2B). For the Algerian mouse I could only assess differences in survival in traps with or without nests in November (no individual was captured in traps with nests during March or July). Survival was lower in traps without nests $(60.0 \pm 7.4 \%, n=45$ captures) than in those with a nest $\left(100 \%, n=11 ; X^{2}=9.76\right.$, d.f. $\left.=1, P=0.0018\right)$. For the common weasel, I also found a similar pattern combining all months, with a lower survival in traps without nests $(66.7 \pm 14.2 \%$; $n=12$ captures) than in traps with nests $(100 \%, n=5)$.

\subsection{Vole captures in the experimental field}

During the study, I made a total of 335 captures of common vole (106 in May and 229 in June) from 227 different individuals (81 in May, 1.31 captures/individual; 146 in June, 1.57 captures/individual). In May 66 individuals were detected once, 8 twice, 5 three times, 1 four times and 1 five times, with a total of 13 spatial recaptures. In June 103 individuals were detected once; 21 twice; 13 three times; 4 four times, 3 five times and 2 seven times, with a total of 25 spatial recaptures. The number of captures and recaptures of male and female voles each month is reported in Table 1, describing the "naïve" vole population (as opposed to the modelled population; see below). The number of females increased considerably from May $(n=47)$ to June $(n=99)$, whereas the numbers of males slightly increased from May $(n=34)$ to June $(n=47)$. Common vole mortality in traps (which all had nests) averaged $2.98 \%(0.9 \%$ in 
May and $3.93 \%$ in June). The few individuals that died in traps were taken into account during the modelling (see Methods).

\begin{tabular}{cccc}
\hline $\mathbf{2 0 1 6}$ & \multicolumn{3}{c}{ Captured and marked $(\mathbf{n})$} \\
\hline & Male & Female & Total \\
May & 34 & 47 & 81 \\
June & 47 & 99 & 146 \\
\hline
\end{tabular}

Table 1. Summary of the "naïve" population of common voles. Number of different males and females captured and marked during the sampling sessions of May and June 2016.

\subsection{Trap saturation and spatial recapture rates}

With the original trapping grid design used in May (Figure $1 \mathrm{~A}$ ), I had 13 spatial recaptures (marked individuals captured in more than one trap during the session) and trap saturation (\% of occupied traps in a given capture occasion) ranged between 0.71 and $8.57 \%$ depending on capture occasions. With the new trapping grid design used in June (Figure 1B), I had 25 spatial recaptures, while trap saturation ranged between 1.61 and $17.74 \%$. Saturation values calculated within the maximum detection area $\left(2.5^{\star} \sigma\right.$ radius $)$ around each trap for the all June capture occasions, are provided in Appendix 1. These indicate that local saturation was never an issue as no trap had a consistently high saturation level over time (throughout the 8 capture occasions).

\subsection{Selection of covariates and model outputs}

During the May capture session, I found no significant difference in $\lambda_{0}$ between sexes and no significant effect of behavior (Table 2). However, I found a significant effect of time (t) on $\lambda_{0}$, which significantly increased between capture 
occasion 1 and 8 (Figure 3; Table 3) in both sexes. I also found that $\sigma$ differed

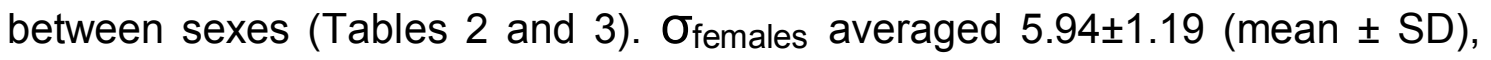
while $\sigma_{\text {males }}$ averaged $11.49 \pm 1.49$ (Table 3 ), indicating greater movements and home ranges in males than in females. The density estimate, $D$, of females (96.22 \pm 36.41 individuals per ha) was almost twice that of males $(46.70 \pm 8.66$ individuals per ha), revealing a highly skewed sex-ratio (Table 3). 

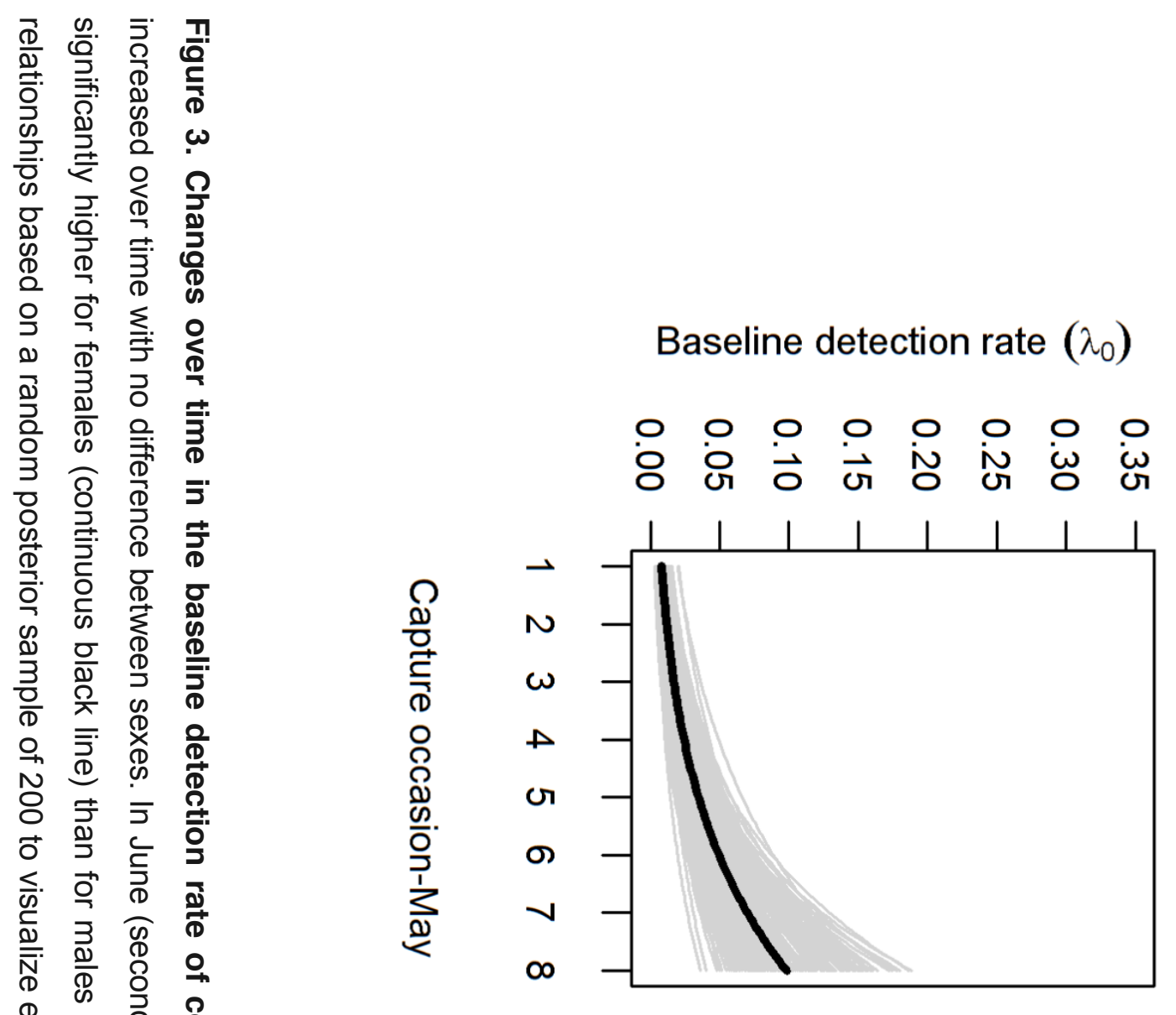

$w$
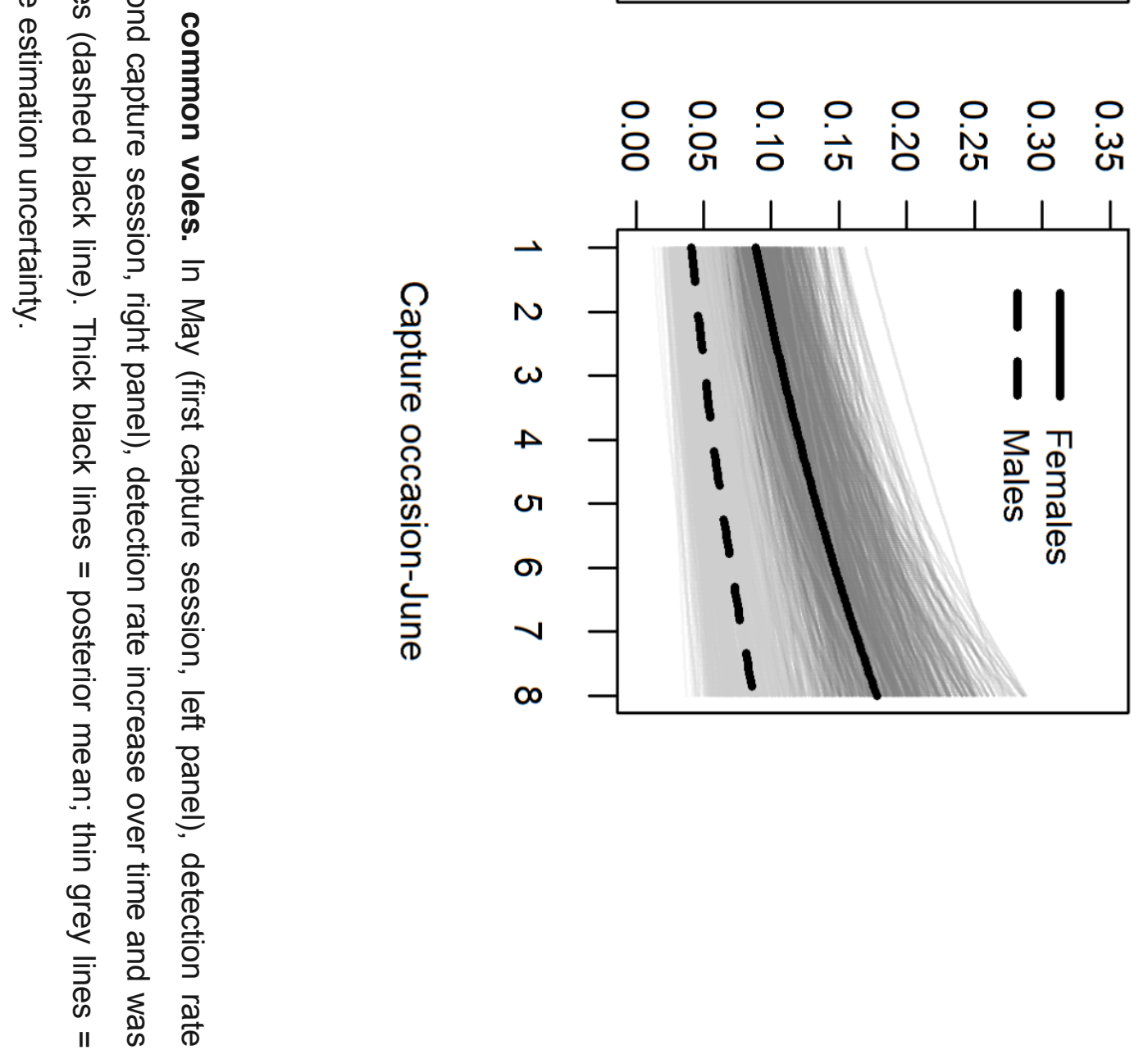


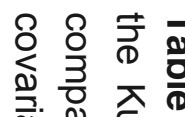

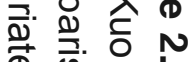

芷

으 중 음

ฏ

ब.

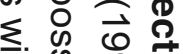

₹ 음 $₫$ 후

巻 3 응 욱

흠윰웅

융 Ð

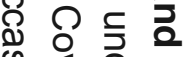

ㅁํㄱ ฏ 응

事茮

D 0 O

蛋的鱼产

$\propto \frac{D}{D}$

उ ॥ 일

응 음

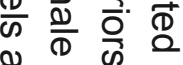

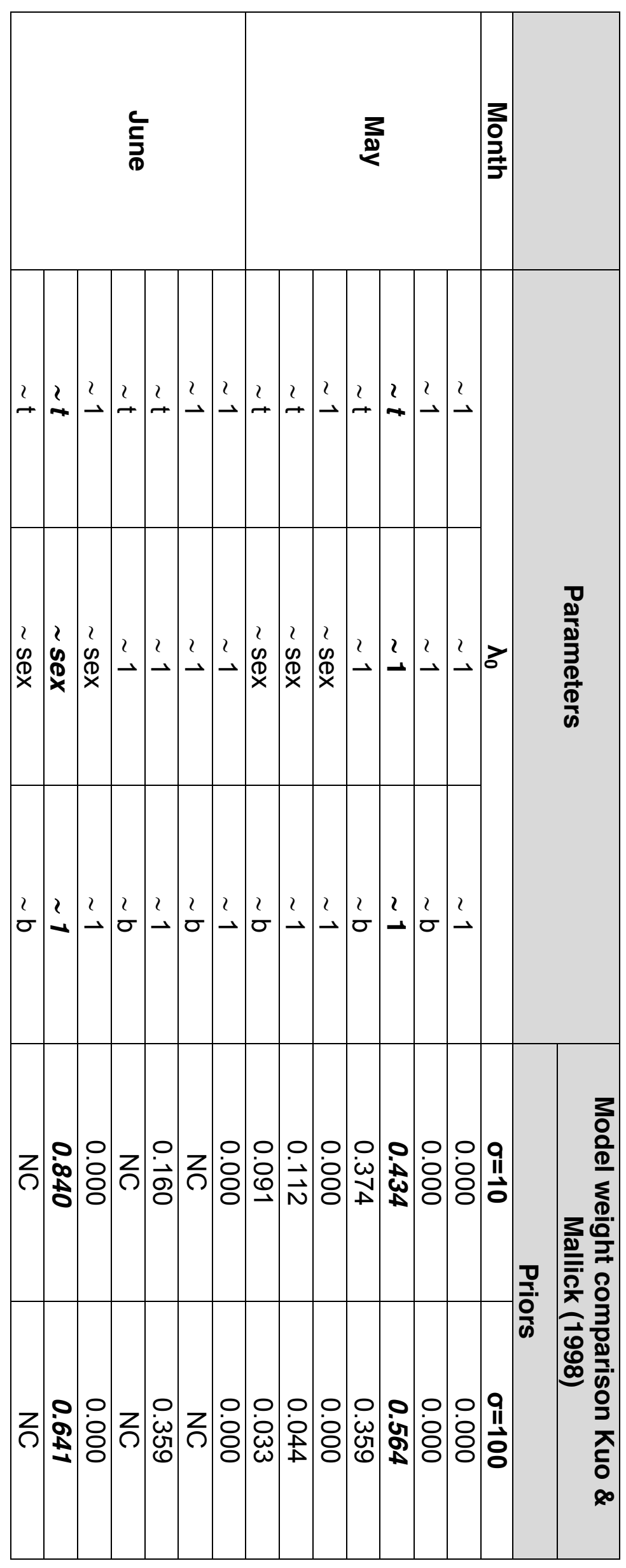

윽

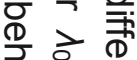

등 긍

요 ه

으 $\overline{\mathbb{1}}$

产要

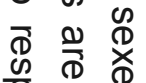

응

勇

웅 훙

$\cong$

동 을.

은. 응

을 을

$\therefore \sum_{\mathbb{\Phi}}$

II 穴

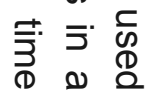




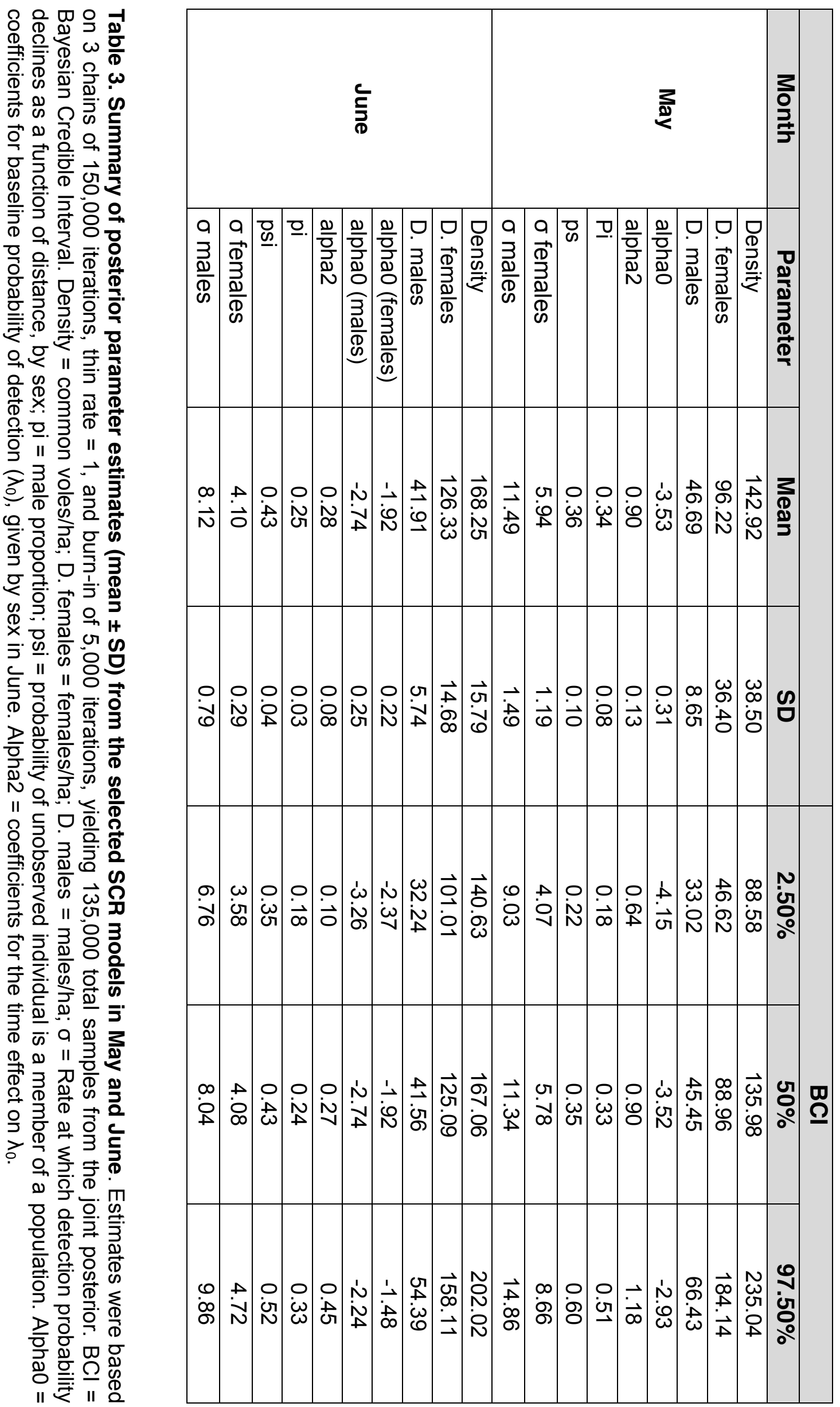


During the June capture session, I could not test for a possible effect of behavior on $\lambda_{0}$ (models including behavior could not converge; Table 2). I again found that $\lambda_{0}$ increased over time (on the first capture occasion of June, $\lambda_{0}$ was similar to the one of the last capture occasions of May and continued to increase over time; Figure 3). Unlike in May, $\lambda_{0}$ differed between sexes, being greater for females than for males (Figure 3; Tables 2 and 3). As for June, I found that $\sigma$ differed between sexes, being almost double for males $\left(\sigma_{\text {males }}=\right.$ $8.12 \pm 0.79)$ than for females $\left(\sigma_{\text {females }}=4.10 \pm 0.29\right.$; Fig 4$)$. Assuming a circular range around the activity center (Royle et al., 2014) of an individual vole, the home range of males $\left(1240 \mathrm{~m}^{2}\right)$ was four times larger than that of females (316 $\left.\mathrm{m}^{2}\right)$. The density (D) estimate was 3 times larger for females $(126.33 \pm 14.68$ individuals per ha) than for males $(41.91 \pm 5.74)$ (Table 3$)$. A graphic representation of density across the state-space area, $S$, and trapping grid is shown in Figure 5. 


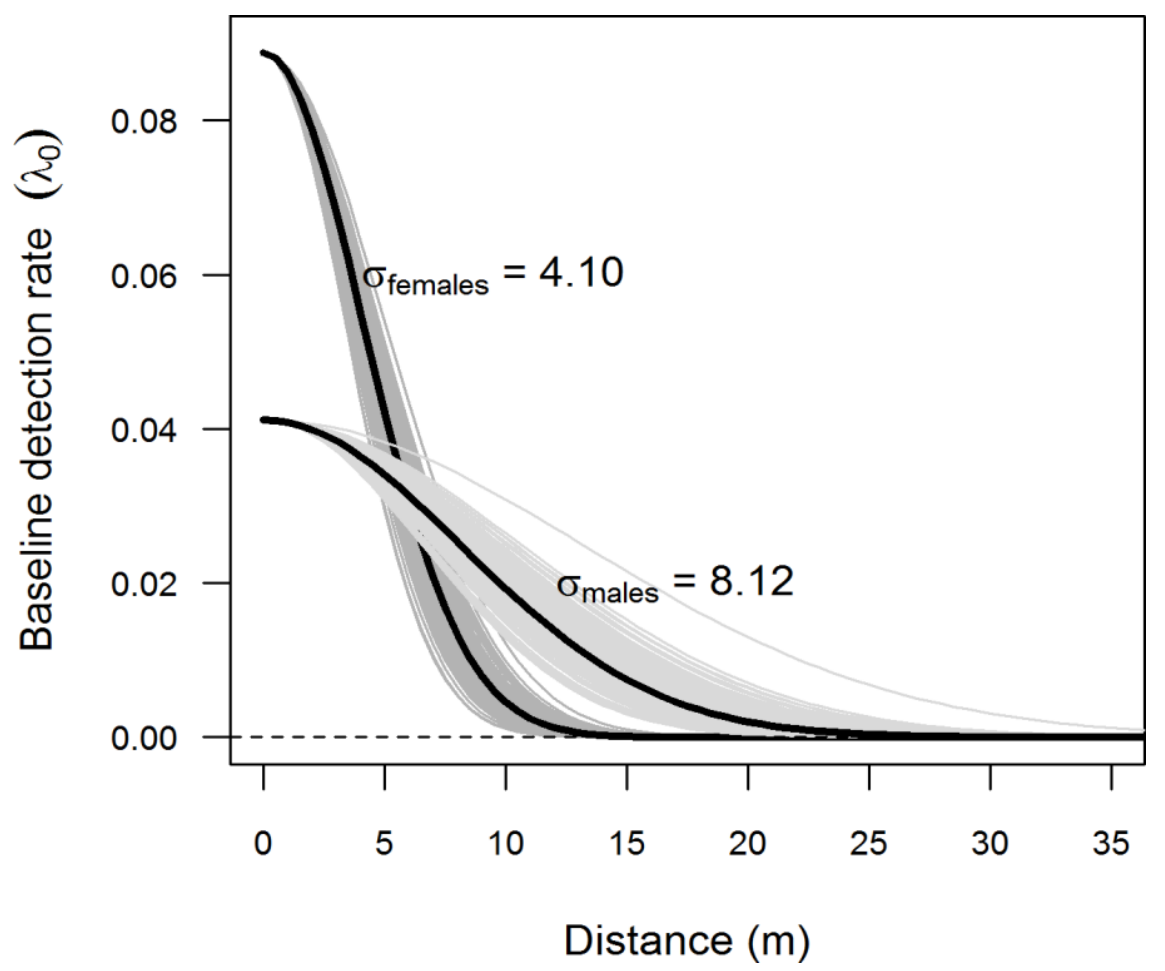

Figure 4. Relationship between the trap to activity center distance $(m)$ and the detection rate of male and female common voles. The model outputs are for the primary capture session of June. The baseline probability detection $\left(\lambda_{0}\right)$ was significantly greater for females than for males, while the rate at which detection probability declined as a function of distance ( $\sigma$ parameter) was c. 2 times greater for males than for females, indicating greater movements and home ranges. Thick black lines = posterior mean; thin grey lines = relationships based on a random posterior sample of 200 to visualize estimation uncertainty). 


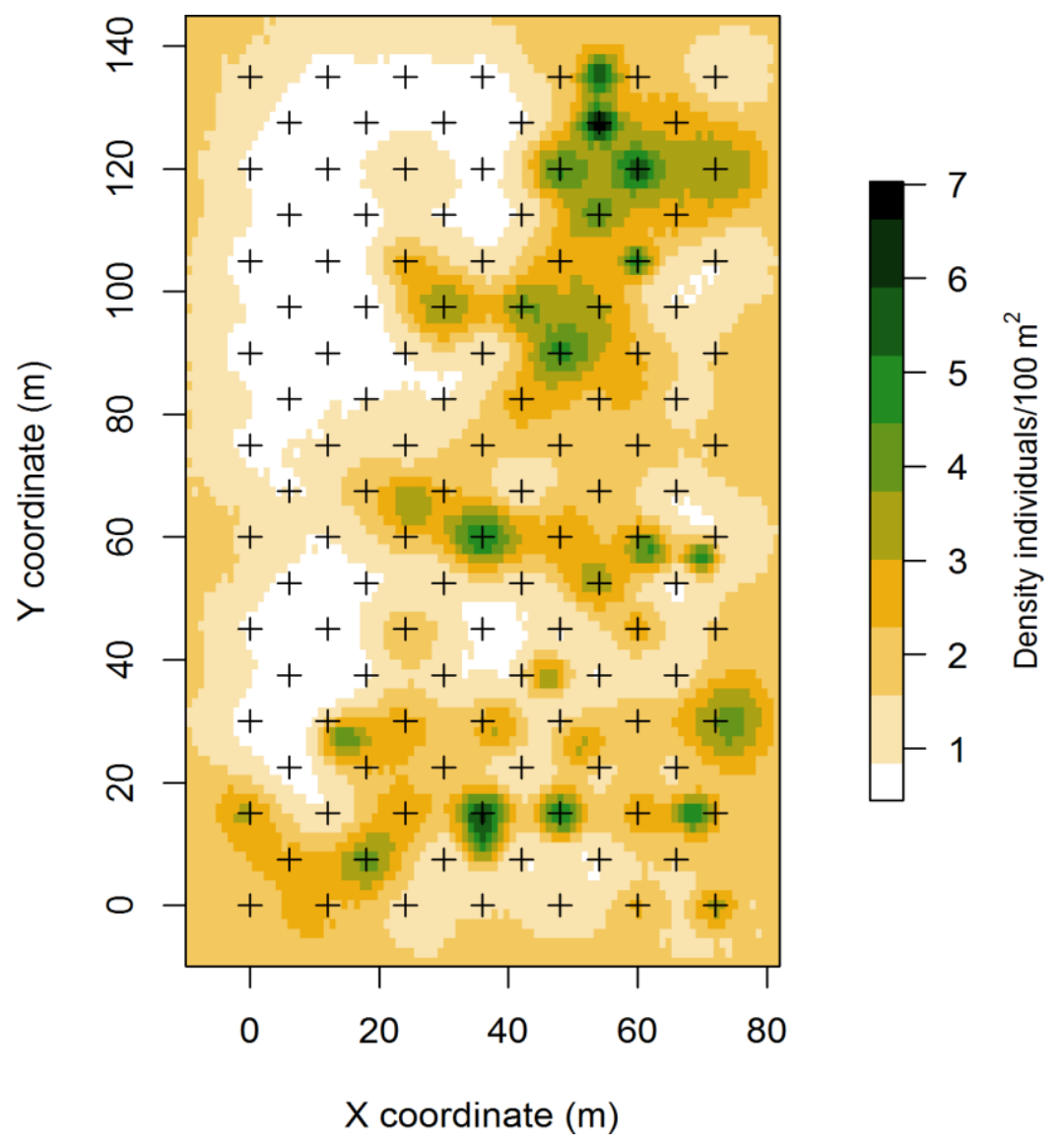

Figure 5. Predicted distribution and mean density of activity centers of common voles. The model outputs are for June. The state-space region covers $25740 \mathrm{~m}^{2}$, each pixel is of $1.45 \mathrm{~m}^{2}$ and the scale is in individuals $/ 100 \mathrm{~m}^{2}$. Black crosses show the trap array, which covered $9720 \mathrm{~m}^{2}(72 \times 135 \mathrm{~m})$.

Simulation analyses using population data from the June model showed that changing the trap configuration from the one used in May to the one used in June (with a reduction in minimum distance between traps from 12 to 9.6-m but using the same theorical vole population) improved the precision of the parameters (population size) and $\sigma$ (movement; see Appendix 1). The reduction in root-mean-square errors (RMSEs) was particularly marked for the parameters estimated for females. This was because females had a lower $\sigma$ 
than males (Appendix 1) and for these, the minimum distance between traps was initially too large, resulting in fewer captures and spatial recaptures. 


\section{Discussion}

\subsection{Trapping mortality}

I first designed and tested a way to reduce mortality during trapping, by adding a nest to a single-catch trapping device (standard Sherman trap). I favored the use of modified Sherman traps, rather than Longworth traps, in this study for two main reasons: 1) their practicality and effective trapping mechanism and 2) their reduced small mammal mortality due to the improvement of adding a nonmetal nest box (mortality that was overall lower than that observed in other studies using standard Longworth traps (Anthony et al., 2005)).

Mortality of small mammals in such traps can be very high, compromising our ability to follow individually marked individuals (Stephens \& Anderson, 2014). I have shown that using a P.V.C nest with food and bedding significantly reduced trapping mortality in common voles. Overall mortality was reduced 5fold, but this effect was more pronounced in November and in March, when trapping mortality was also the greatest. By contrast, in July, trapping mortality was lower and similar in nests with or without traps (Figure 2). During our study, temperatures (mean [range: Min—Max]) were colder in November $2016\left(5.8^{\circ} \mathrm{C}\right.$ [-4.6 ${ }^{\circ} \mathrm{C}$ to $\left.\left.21.7^{\circ} \mathrm{C}\right]\right)$ and March $2017\left(8^{\circ} \mathrm{C}\left[-4.05^{\circ} \mathrm{C}\right.\right.$ to $\left.\left.24.9^{\circ} \mathrm{C}\right]\right)$ than in July 2017 $\left(20.8^{\circ} \mathrm{C}\left[4.02^{\circ} \mathrm{C}\right.\right.$ to $\left.\left.35.4^{\circ} \mathrm{C}\right]\right)$, with some frost occurring during the former two months. This suggests that vole mortality was higher in colder weather conditions (Jackson et al., 2001), in particular during frost, and that the addition of a P.V.C nest box minimized the risk of mortality by hypothermia. The characteristics (i.e. thick plastic walls) of our P.V.C nest boxes allows the captured individual to wait in an isolated warm and dry chamber until trap 
checking. Mortality could also increase during very hot conditions or under heavy rain because of trap flooding (Dizney et al., 2008; Anthony et al., 2005); I did not record any mortality due to heat or rain during our study, but their effect on survival with nests should be tested in future work. In another study in wet climate, mortality was reduced by protecting traps with a rain shield (Dizney et al., 2008), although problems still arose when excessive rain flooded the bottom of the traps. Our study shows that the addition of a P.V.C nest box also improved the survival in traps of other species, such as the wood mouse, the Algerian mouse or the common weasel. These observations suggest that, under broadly similar weather conditions (same month), a coupled nest box should also perform well with these other small mammal species. However, a larger sample size would be desirable to test more rigorously the effect of the nest box on the survival of the latter two species, taking into account possible variations in microclimatic conditions at the field margin level.

\subsection{Trap saturation and spatial recapture rates}

The number of traps and the frequency of visits to retrieve trapped individuals are key aspects to avoid trap saturation, i.e. a lack of active trapping devices available for new captures. In the present study, I initially set up trapping devices in pairs to avoid potential saturation. However, in May, with a density of about 140 voles per ha (Table 3 ), I always had less than $10 \%$ of occupied traps during a given capture occasion, so I was well below the previously recommended threshold of trap saturation of 60\% (Distiller \& Borchers, 2015) or 86\% (Efford et al., 2009). This allowed me to switch the design from pairs of traps per capture point to single traps (Krebs et al., 2011), and to modify the 
grid (Figure 1) to try to increase spatial recapture rate, improve model performance and reduce uncertainty around parameter estimates.

In SCR studies, the spatial organization of traps and overall grid design should aim at increasing spatial recaptures (i.e. individuals captured in more than one trap in different occasions), to better inform about animal movement, and improve the accuracy of the model outputs. To set the distance between traps, a distance smaller than $2 \sigma$ is recommend (Sollmann et al., 2012; Sun et al., 2014). I didn't have any a priori knowledge of vole movement $(\sigma)$ in our study area. After the session of May, spatial recapture rate was low (c. $5 \%$ ) and the distance between traps appeared too large for an accurate estimation of $\sigma$ (Figure 4). By adjusting the grid design (i.e., placing one trap in the middle of the initial square formed by four equidistant traps (Figure 1B), I reduced the minimum distance between traps from 12 to $9.6 \mathrm{~m}$. With the new configuration of traps deployed in June, I obtained more captures (229 vs 106 in May), and more spatial recaptures (25 vs 13 in May). Overall, I also obtained measurements of $\sigma$ and with a reduced $\mathrm{BCl}$ in June compared with May (Table 3). The results of our simulations (Appendix 1) indicated that the change in trap configuration contributed to improving the parameters estimates (reducing $\mathrm{BCl}$ ). This was particularly evident for the parameter estimated for females (Appendix 1), which had a lower sigma than males, and for which the minimum distance between traps was too large in May (Appendix 1) (Sun et al., 2014).

When trap spacing exceeds the average home range of a species there would be no or few spatial recaptures, as most individuals would be captured in a single trap, nearest their activity center (Dillon \& Kelly, 2007). A primary aim when applying SCR models should be to achieve as many spatial recaptures as 
possible. Trap saturation in June increased to a maximum of about $18 \%$, when traps were reduced from 140 to 124 , but this was not an issue as saturation was kept to low levels. Saturation measured at the scale of an average area of maximum detection for a common vole was also not an issue. I never found a consistently high saturation level in any given trap throughout the 8 capture occasions (Appendix 1). This is in agreement with a previous simulation study (Efford et al., 2009) that showed that the multi-catch model yielded nearly unbiased results for saturation levels below $86 \%$, even when animals were clustered. If density increases and trap saturation becomes an issue, then the time interval between checking traps can be reduced and/or the number of traps increased.

\subsection{Insights from covariates retained in SCR model selection}

During both May and June, the best selected model included a $\lambda_{0}$ that increased over time (Table 3). An important component of capturing a particular individual is the chance that the trap will be encountered. For instance, the encounter rate can change due to the number of traps placed during the trapping period and their spatial placement (Gurnell \& Langbein, 1983). In our study, I observed a clear lack of detection during the first capture occasions, and a progressive increase in $\lambda_{0}$ throughout the following capture occasions. It is worth noting that in May I set up new, previously unused, Sherman traps and nests (Appendix 1). Previous works showed that odors left by previous occupants can affect subsequent occupancy, and traps with no previous occupant had lower capture rates (Brouard et al., 2015). The olfactory cues in and around a trap can

strongly influence trap detection and entry by small mammals (Stoddart \& Smith, 1986). The complete absence of biological odors in new traps may 
therefore explain an initial neophobic response in voles, which typically show an active preference for substrates tainted with chemical signals from conspecifics (Luque-Larena et al., 2001; Luque-Larena et al., 2002; Luque-Larena et al., 2002). We had no evidence for common voles to be trap-shy (less likely to enter a trap after an initial capture) or trap-happy (the opposite), at least in May when the behavioral effect could be included in the models. Hence, I suggest that the increased detection rate over time was mainly due to a progressive increase in olfactory cues left by the previously captured individuals (faeces, urine) that subsequently increased both trap detection and trapping rate.

In June, $\lambda_{0}$ continued to increase over time, but also turned different between sexes (Figure 3): females ended up with a higher $\lambda_{0}$ than males. This difference may be because female voles often have a socially dominant status (Ylönen et al., 1990) and/or are more territorial than males (Bujalska \& Saitoh, 2000), but I do not have a clear explanation for such differences in detection rates between sexes. However, our result highlights that differential detectability between sexes shouldn't be overlooked, as "naïve" estimates of male and female density could be consequently biased (i.e., in our case ignoring differences in $\lambda_{0}$ or $\sigma$ would result in an underestimated male density, because males were less detectable than females).

Interestingly, I found a marked difference in $\sigma$ parameters between sexes during both months (Figure 4; Table 3). The estimated $\sigma$ for males was twice that of females, indicating that males moved further away from their activity center (burrow, colony) than females. SCR thus showed that females had smaller home ranges, were less mobile, but were still more likely to be detected. Our home range estimates $\left(1240 \mathrm{~m}^{2}\right.$ and $316 \mathrm{~m}^{2}$ for males and females, 
respectively) assume a circular home range with $2.5^{\star} \sigma$ radius around the activity center, which may be not very realistic in some cases (e.g. when the activity center is located in a field margin instead of inside a field). Nevertheless, these estimates are consistent with those reported in other studies conducted on voles, like field voles Microtus agrestis, in which males (range 87-1037 $\mathrm{m}^{2}$ ) had larger home ranges than females (range 31-225 $\mathrm{m}^{2}$ ) (Borowski, 2003). Other studies on common vole home range reported a mean home range-size of $200 \mathrm{~m}^{2}$ (Jacob \& Hempel, 2003), $145 \mathrm{~m}^{2}$ (Mackin-Rogalska, 1981) or $125 \mathrm{~m}^{2}$ (Briner et al., 2005), without noticeable differences between sexes (Jacob \& Hempel, 2003).

During the breeding season, females likely decreased their movements, while males increase theirs, probably in search of females to mate with. For instance, similar behavior of active males searching females covering extensive areas at the beginning of the breeding season is also seen in other rodents such as meadow voles M. pennsylvanicus (Ostfeld et al., 1988; Sheridan \& Tamarin, 1988), and the white-footed mice P. leucopus (Wolff \& Cicirello, 1990). Understanding individuals' movements in a given season or habitat is fundamental in ecological studies, and SCR method enables the integration of explicit movements with models that incorporate density and other population characteristics (Ergon \& Gardner, 2014; Schaub \& Royle, 2014). 


\subsection{Model outputs}

The SCR modelling allowed me to obtain a precise estimate of common vole density as well as information about how they were distributed within the study field (Figure 5). The species is colonial (Jacob et al., 2014), and this is clearly evident from the density map provided as a model output. The model results also revealed a population sex ratio that was skewed towards females (with ratios of 2 and 3 females to 1 male in May and June, respectively). These estimates are accurate because they take into account differences between sexes in baseline detection rates and movements, two factors that can contribute to bias naïve estimates of common vole population sex ratio (Bryja et al., 2005). The sex ratio is a key parameter for population studies but is difficult to reliably estimate it in voles. The SCR approach offers a credible and accurate estimate to investigate variation in this key population parameter. To ensure accurate estimates of sex-ratio, it is necessary to consider potential differences in movement between sexes, and to carefully adjust the trap configuration (minimum distance between traps) to the movement parameter $\sigma$ (sigma) of the sex that moves the less (Appendix 1). The application of SCR models allowed me to precisely estimate $N$ (population size) and $D$ (density), two crucial parameters for population dynamic or management studies (Sutherland et al., 2014; Krebs \& Boonstra, 1984). In addition, SCR models allowed to describe important spatial characteristics of study species, revealing a different use of space by females and males (i.e. different home ranges) and provided a precise mapping of activity centers, allowing to clearly identify active burrows and colonies. 


\section{Conclusions}

SCR are relatively new models (Royle et al., 2014; Efford, 2004; Royle et al., 2017; Efford et al., 2009) that allow overcoming common challenges while providing a statistically coherent framework to estimate abundance and density. SCR models are increasingly used in ecological and conservation studies of mammals worldwide but are still underused for studying small mammals like burrowing rodents (Krebs et al., 2011; Ergon \& Gardner, 2014; Sutherland et al., 2014). After innovating, testing and adjusting the trapping methodology to our model species, I have highlighted the great potential and broader applicability that SCR modelling offers for studying voles. This should encourage further application of this methodology for the study of voles and other small mammals in order to get precise, reliable and accurate measurement of density and population structure, and to improve our understanding of their population ecology. To ensure accurate results, basic knowledge is however necessary to optimally design the study, and a pilot study may be particularly useful in the absence of detailed a priori knowledge on the species of interest. In order to successfully apply SCR to the study of small mammals such as voles, particular attention must be paid to: 1) reduce mortality in traps; 2) assess the possible effects of residual odors in traps (or the lack of these, when using new, unused traps) on capture rates; 3) monitor levels of saturation and adjust the number of traps or duration of capture occasion bouts accordingly; and 4) carefully adjust the trap configuration (minimum distance between traps) to the movement of the species, and more specifically to the class of individual that moves the less (like in our case females) in order to get more precise information on population composition (e.g. sex-ratio). 


\subsection{Chapter "2"}

Abundance indices validation

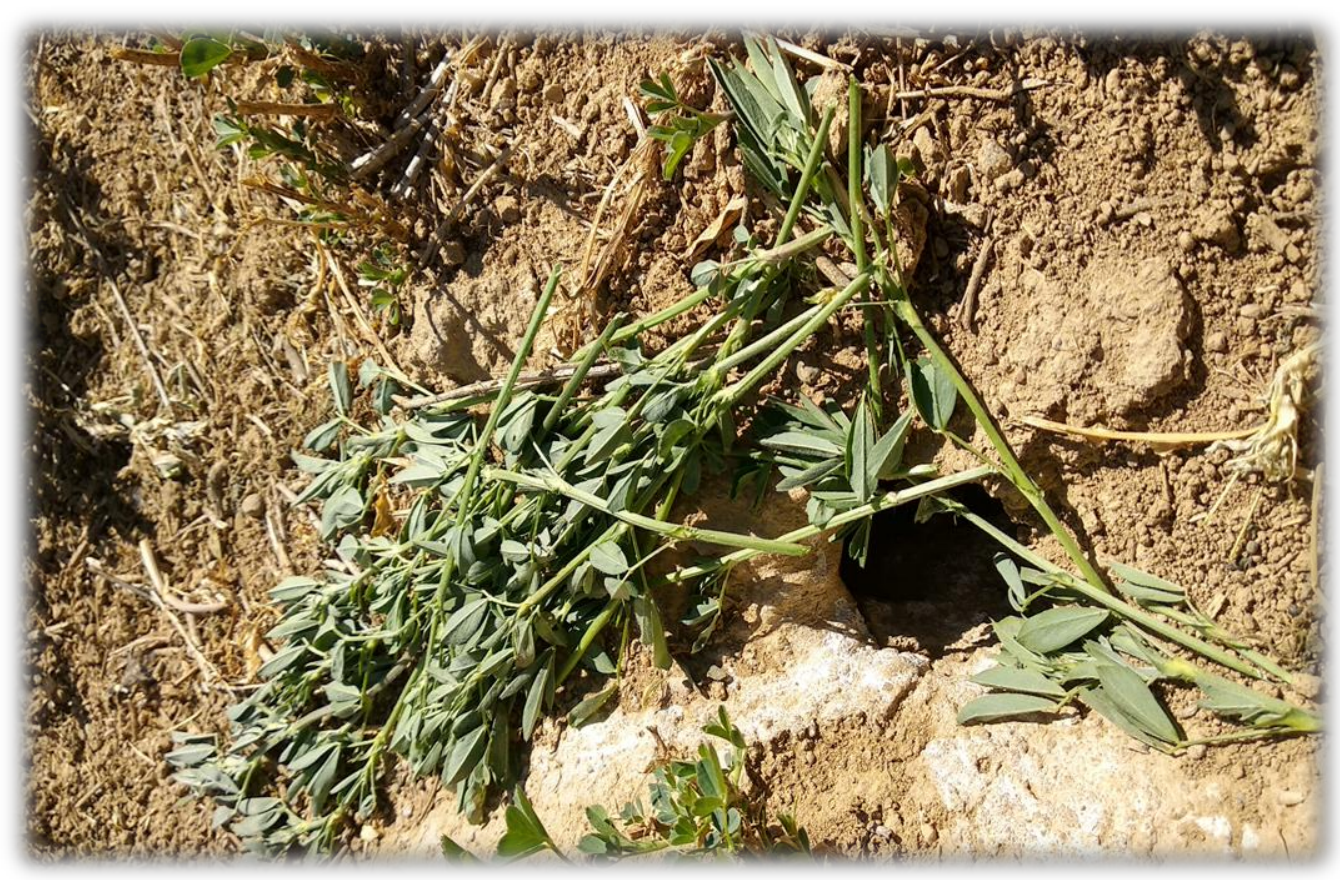




\section{Using spatial capture-recapture modelling to calibrate indirect abundance estimates for the large-scale monitoring of a farmland rodent pest}

\section{Abstract}

A reliable estimation of rodent abundance is required to effectively monitor populations, study their ecosystem role or implement timely management actions when rodents become pests that impact on agriculture or public health. Live-trapping, capture-mark-recapture (CMR), and spatial capture-recapture (SCR) modelling provide to date the most accurate means of estimating the abundance of small burrowing rodents such as common voles (Microtus arvalis), a widespread facultative farmland pest in Europe. Large-scale repeated monitoring however requires easier, cheaper and less time-consuming methods, readily provided by the use of vole activity signs (VAS). Here I compare vole abundance estimates derived from SCR and VAS within the same $\sim 1.2$ ha experimental alfalfa field in NW Spain, monitored monthly over 20 months (July 2016 to February 2018), during a moderate vole fluctuation in abundance (ranging from $4.0 \pm 1.4$ voles ha $^{-1}$ in February 2018 to $112.97 \pm$ 24.5 voles ha-1 in August 2016). Vole density was measured and mapped within the whole $\sim 1.2$ ha area while VAS was assessed along 7 transects (each $1.5 \mathrm{x}$ $99 \mathrm{~m}, 148.5 \mathrm{~m}^{2} /$ transect) located inside the alfalfa field $(\mathrm{n}=5)$ and in field margins $(n=2)$. A combination of presence of burrows with at least one of three vole activity signs (recent soil excavations, fresh fecal droppings and freshly cut vegetation, all within the burrow inlets) predicted common vole density in the 
experimental field within our range of densities. VAS from the transects within the field provided better predictions than those measured in field margins. I also found that the association between vole density and VAS varied seasonally, with fewer VAS detected than expected from density during spring-summer months as compared with autumn-winter months. This seasonal variation was in part explained by a lower detectability of VAS with increasing vegetation height, and with reduced vole activity (as estimated from vole detectability in traps). Overall, I show that vole activity sign assessments provide reliable estimates of vole density, at least up to 113 voles ha ${ }^{-1}$ ), but that translation of vole density (vole's ha ${ }^{-1}$ ) from the indirect vole abundance estimate (VAS) should be season specific.

Keywords: Capture-mark-recapture (CMR), basal probability of detection, movement, indirect methods, abundance, activity signs, seasonality, common vole, Microtus arvalis 


\section{Introduction}

Small mammals are widely distributed and are often considered a central component of many ecosystems (Gauthier et al., 2011; Krebs et al., 2011). Some species undergo periodic or irregular fluctuations of abundance (Lambin et al., 2006; Tkadlec \& Stenseth, 2001). For instance, in northern and central Europe, multiannual population cycles are a common feature and occur with a period of 2-5 years (Cornulier et al., 2013). Some small rodents such as the common vole (Microtus arvalis) cause important crop damages and economic loss during outbreaks in farmland areas across Europe (Jacob \& Tkadlec, 2010). In addition, during outbreaks, common voles can act as amplifier in the environment of zoonotic diseases that have important public health implications (Han et al., 2015; Luque-Larena et al., 2017, 2015; Rodríguez-Pastor et al., 2017). For all these reasons, there is strong interest in monitoring abundance variation in vole populations, for which a reliable estimator is required. Regarding pest management, the method used should also be easy, simple and cheap, in order to allow regular monitoring at large spatial scales (Delattre et al., 1990; Jareño et al., 2014). As knowledge of the studied species increases and new methods become available, it is important to review and re-examine the reliability of the methods utilized to estimate abundance (Embleton \& Petrovskaya, 2013; Lisická et al., 2007), in order to improve monitoring, forecasting and decision-making.

Small mammals are difficult to detect because they are often nocturnal and elusive, predominantly as an anti-predation method (Capizzi \& Luiselli, 1996) and/or because they live underground (Fauteux et al., 2018). This 
difficulty is partly alleviated when estimating abundances through the use of different capture methods, such as capture-mark-recapture (CMR), which can provide accurate and reliable density data through detailed information on captured individuals (species, sex, reproductive status or condition) and estimates of their detectability. CMR has been used for many years to estimate animal population's density (Otis et al., 1978; Seber, 1982; Pollock et al., 1990; Amstrup et al., 2005), including common vole populations in Castilla-y-León region (Jareño et al., 2014; Romairone et al., 2018). Recent developments of this methods include spatial capture-recapture models (SCR), which provide better density estimates since they make use of the spatial locations of the encountered individual (i.e. taking into account individual heterogeneity in encounter probabilities due to the location of the individuals' home range or activity center relative to traps locations) (Efford, 2004; Royle \& Young, 2008; Borchers \& Efford, 2008; Gardner et al., 2009; Royle et al., 2014, 2017). These methods are well suited for estimating rodent abundance and are currently considered the most robust from amongst the available methods (Gerber \& Parmenter, 2015; Krebs et al., 2011; Parmenter et al., 2003; Romairone et al., 2018). Additionally, SCR models also inform on movement (home range size) or basal probability of detection in traps (see Romairone et al., 2018 for more details). Estimating these latter parameters can inform on possible variations in movements or basal detection rate or in time (e.g. seasonal variations, densitydependence; Casula et al., 2018).

Methods like CMR or SCR are, however, time-consuming (requiring several days of continuous trapping to mark and recapture individuals; Tellería, 2004; Witmer, 2005) and expensive, requiring expertise and equipment (Batzli 
et al., 1983, Henttonen et al., 1987; Krebs et al., 1995). These time and financial constraints make them less suitable when continuous and large-scale monitoring of small mammals is required. In addition, when using methods that involve live captures, field workers may face risks to their health as some diseases are transferable (i.e. zoonotic diseases) meaning that, when possible, it is preferable not to handle animals (Gervais, 2010).

Indirect methods estimate the abundance of a species without capture. These methods are cost-effective (Thompson et al., 1998), quick and easy to implement and thus enable monitoring to be carried out over large scales (Giraudoux et al., 1995; Miñarro et al., 2012), but are less precise, and prone to various sources of errors (Telleria, 2004; Witmer, 2005; Güthlin et al., 2014). For example, differences in the persistence or detectability of signs among seasons or habitats can lead to biased estimates (Güthlin et al., 2014). There exist a large variety of methods available to indirectly measure abundance, and their appropriateness depends on the study species as well as the specific time and landscape (Gompper et al., 2006). Vole abundance indices based on activity signs (VAS) such as the presence of fresh droppings, active burrows or vegetation clippings, can provide a suitable alternative to trapping and marking animals (Gervais, 2010; Jareño et al., 2014). Multiple studies have highlighted their usefulness for estimating vole abundance (Delattre et al., 1999, Lambin et al., 2000; Wheeler, 2008; Terraube et al., 2011; Jareño et al., 2014), although in some cases, indirect methods proved inaccurate (Gervais, 2010). A limitation to abundance indices is that, despite their widespread use, they are often uncalibrated (i.e. they only provide a relative abundance, not an absolute one that could be compared across different contexts; Anderson, 2003, Krebs et al., 
2012), therefore, they are less precise than trapping methods and may be subject to biases.

In recent decades, the common vole has colonized the agricultural landscapes of Castilla-y-León (NW Spain), invading c. 5 million ha of farmland where vole outbreaks are now commonplace, causing conflicts with agricultural production (Luque-Larena et al., 2013). Subsequently, since 2007 the Ministry of Agriculture and Livestock (CAG) of the regional government of Castilla-yLeón (JCyL), has worked on the development of Integrated Pest Management (IPM) strategy applied to crop protection against common vole derived risks. Within the scheme IPM, a large-scale monitoring of common vole populations is a basic and fundamental support tool for decision-making. The main objectives of this monitoring program, coordinated from "Observatorio de Vigilancia y Control de Plagas Agrícolas de Castilla y León" (ITACyL, CAG, JCyL), are to: (i) assess the status and temporal trends of common vole populations, (ii) identify early events of vole demographic increases, and (iii) establish and define risk zones and thresholds for actions. Predominantly, the monitoring is based on indirect methods, like VAS (i.e. recent burrowing, fresh droppings or fresh plant clipping remains) evaluated along transects all over around the main farmland areas of Castilla-y-León in crop plots (e.g. barley, alfalfa and wheat fields), and reservoir habitats (e.g. meadows, fallow fields and field margins; Jareño et al., 2014; Rodriguez-Pastor et al., 2016). The VAS approach provides for each sampling an index of vole abundance (\% of units with positive VAS). Indirect approaches are complemented in some recognized problematic areas with livetrapping, to obtain more detailed information on vole abundance, population structure (sex-ratio) and reproductive status of females. Thus, the regional 
monitoring program provides useful information to estimate different risk threshold for different zones of Castilla-y-León.

For the optimization of the decision-making process, a continuous improvement and calibration of the methods used for species population estimates are required and is fundamental for any IPM. In this chapter, the main objective is to validate and calibrate the VAS method through comparison with SCR derived density estimates. For this purpose, I monitored the fluctuations in vole abundance in a 1.2 ha experimental field using both VAS and CMR over two years. Vole density was measured by using the most accurate method available to date (using SCR modelling with CMR data) in order to relate the VAS index (\% sampled units with signs of vole presence) to vole density (number of voles per ha) at field and transect levels. Additional questions were explored: (i) which combination of vole activity signs (burrows, clipping and/or droppings) better predicted vole density? and (ii) what is the influence of season, vegetation height and vole behavior (SCR model outputs) on the relationship between VAS and vole density? 


\section{Material and methods}

\subsection{Study area and design}

The study was conducted in a 1.2 ha experimental field located in Soto de Cerrato $\left(41^{\circ} 94^{\prime} \mathrm{N}, 4^{\circ} 42^{\prime} \mathrm{W}\right)$, Castilla-y-León. The surrounding area is characterized by an intensive agricultural landscape dominated by cereals and alfalfas (Jareño et al., 2015; Rodríguez-Pastor et al., 2016). The experimental field is fenced with a $2.5 \mathrm{~m}$ tall wire mesh perimeter that prevents public access while allowing small mammals to move through. In spring 2015, the field was planted with alfalfa (Medicago sativa, "Aragón" type), and was not irrigated throughout the study.

The two methods for estimating vole abundance (CMR and VAS) were carried out from July 2016 until February 2018 in the experimental enclosure and repeated monthly at the same locations (except in March 2017, when VAS was not measured due to fieldwork constraints). Vole live-trappings sessions for CMR lasted six consecutive days, and capture sessions were separated by a three-week interval without trapping. A total of 20 trapping sessions were carried out to measure vole density (see below for details). The first day of each session was used to set up the traps, which were active early in the evening, and followed by five days of captures. VAS surveys were conducted immediately after the trapping session (in the same week). Concomitantly to VAS assessments I also collected monthly data on vegetation characteristics at three random locations within the experimental enclosure. I recorded the following characteristics: 1) vegetation height $(\mathrm{cm}), 2)$ vegetation cover (scored from 0 to 4 for the following ranges: $0-20 \%, 20-40 \%, 40-60 \%, 60-80 \%, 80-$ 
$100 \%$ ) and 3) vegetation status (coded as 1: predominantly dry, 2: heterogeneous, 3: predominantly green).

a.

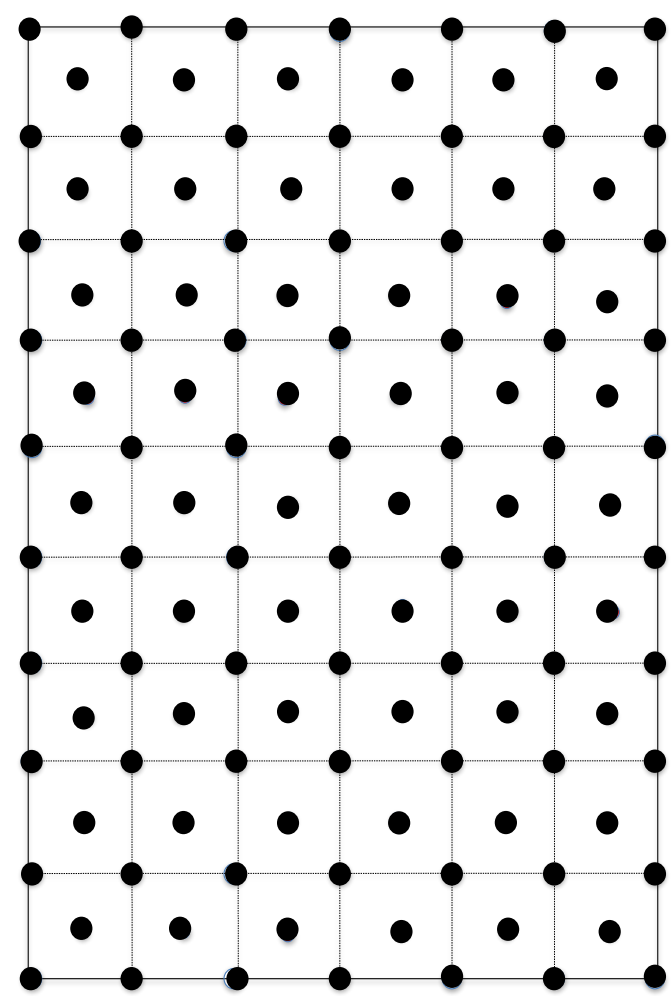

b.

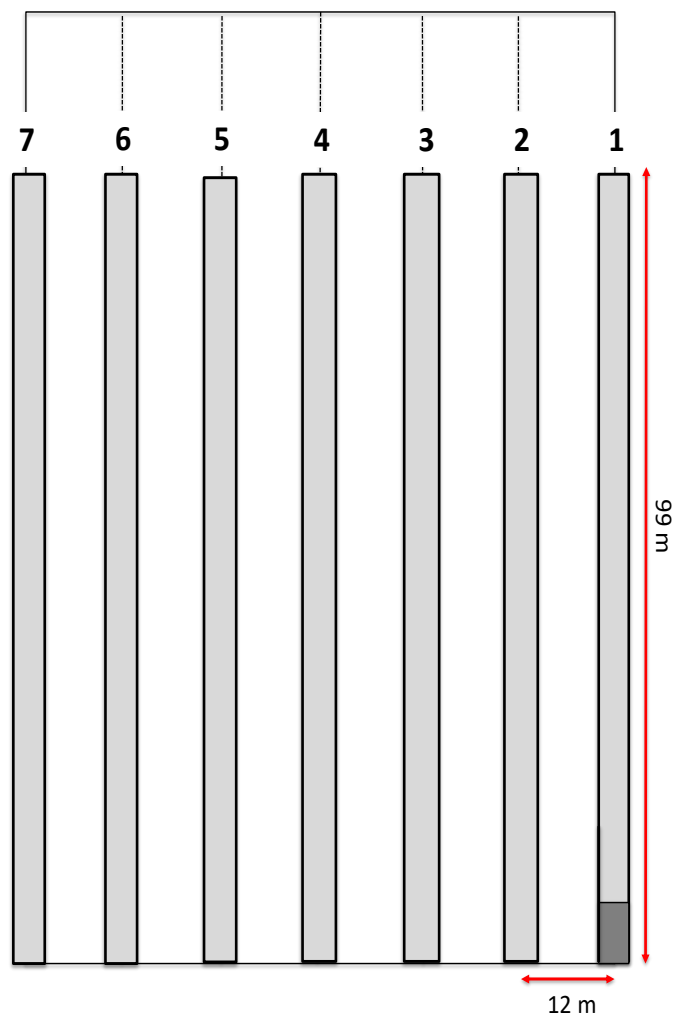

Figure 1. Sampling designs used for CMR and VAS methods. (a) Trapping "grid" used for CMR sessions, which included a total of 124 traps (black points) and a grid size of $72 \times 135 \mathrm{~m}$. (b) Linear transect bands $(n=7)$ used to record the VAS; transects 2 to 6 are located within the alfalfa field; 1 and 7 are located at the field margins. Presence signs of voles were searched along $99 \mathrm{~m}$ straight transect sections (grey bands) of $1.5 \mathrm{~m}$ width, each divided into 33 blocks of $3 \mathrm{~m}(\mathrm{n}=33$ per transect section; darker grey color).

\subsection{Density estimates}

\subsubsection{CMR trapping sessions}

Live trapping was conducted using a trapping grid of $72 \times 135 \mathrm{~m}$ (area $=9720$ $\mathrm{m}^{2}$ ) with 124 regularly spaced trapping stations $(n=124,1$ trap per station; 
Figure 1a). I used a traditional CMR method in a permanently marked study grid. All trapping devices were classic Sherman traps $(8 \times 9 \times 23 \mathrm{~cm}$; LFTA Sherman(C) coupled with a P.V.C nest box used to increase the survival rate of captured individuals during the coldest months (Romairone et al., 2018). P.V.C nest boxes were provided with bedding material (paper stripes) and baited with carrot and fodder that provided food and water for trapped animals (Global Diet 2018, Pellet $12 \mathrm{~mm}$, Teklad, Italy). Each trapping device (trap+nest) was covered by an inverted U-shaped concrete block to protect it from heavy rain, direct sunlight and to reduce the impact of frost. Each vole captured for the first time was individually marked with a transponder (Glass tag, BIOGLASS 8625, 2x12mm, Ref: ICAR 941). I recorded the trap location, vole sex and released each individual at its capture site. During subsequent recapture occasions, I recorded vole identity and trap location.

\subsection{SCR modelling}

SCR modelling allows accurate population density estimates (D) and to derive other parameters that can inform about vole behavior and movement. I defined an effective sampling area using a $30-\mathrm{m}$ buffer for creating the state space $(S)$ and used a half-normal detection function for $\sigma$ (see Chapter 1; Romairone et al., 2018 for more detailed information). SCR models were done using secr (see Chapter 1; Romairone et al., 2018).

I used a Maximum Likelihood Estimation (MLE) approach and SCR modeling (using the secr v. 2.9.3 package implemented in R v. 3.1.1; Efford et al., 2016; R Core Team 2014). The modelling simultaneously estimates three structural parameters: (1) population density, (2) the basal probability of 
detection when an animal's home-range center coincided with a trap location $\left(g_{0}\right)$, and (3) a scale parameter sigma $(\sigma)$ - the relationship between detection probability and the distance between an animal's activity center and the capture device (trap position). In addition, the modeling allows testing whether covariates influence the parameters $\sigma$ or $g_{0}$ and should be included to obtain unbiased estimates of density. A model selection allows to specifically test if the parameter $\sigma$ varies according to sex and if the parameter $g_{0}$ varies according to sex, over time (t) or according to vole behavior (b)(see Chapter 1; Romairone et al., 2018 for more details). Therefore, for each monthly CMR session, I first fitted six candidate models that differed only in how these covariates affected $g_{0}$ and $\sigma$ (Table 1). I ranked these models using Akaike's Information Criterion with a second-order correction for small sample sizes (AICc; Burnham and Anderson, 2002). For each monthly CMR, I considered the model with the lowest AICc value to be the best fit for the data and most parsimonious. A summary Table with all (and selected) models for each monthly CMR session is provided in Table 3.

Table 1. Summary of the candidate models used to estimate density (D) for each month using secr. Models vary depending on the covariates affecting $g_{0}$ and $\sigma$.

\begin{tabular}{lll}
\hline Models & & Description \\
& & \\
\hline Model 0 & $\mathrm{D} \sim 1, \mathrm{~g}_{0} \sim 1, \sigma \sim 1$ & Null model \\
Model 1 & $\mathrm{D} \sim 1, \mathrm{~g}_{0} \sim \operatorname{sex}, \sigma \sim \operatorname{sex}$ & Sex differences in $\mathrm{g}_{0}$ and $\sigma$ \\
Model 2 & $\mathrm{D} \sim 1, \mathrm{~g}_{0} \sim 1, \sigma \sim \operatorname{sex}$ & Sex differences only in $\sigma$ \\
Model 3 & $\mathrm{D} \sim 1, \mathrm{~g}_{0} \sim \mathrm{t}, \sigma \sim \operatorname{sex}$ & Temporal variation in $\mathrm{g}_{0}$ and sex differences in $\sigma$ \\
Model 4 & $\mathrm{D} \sim 1, \mathrm{~g}_{0} \sim \mathrm{b}, \sigma \sim \operatorname{sex}$ & Behavior in $\mathrm{g}_{0}$ and sex differences in $\sigma$ \\
Model 5 & $\mathrm{D} \sim 1, \mathrm{~g}_{0} \sim \mathrm{b}+\operatorname{sex}, \sigma \sim \operatorname{sex}$ & Behavior and sex differences in $\mathrm{g}_{0}$ and sex differences in $\sigma$ \\
\hline
\end{tabular}


When $g_{0}$ and $\sigma$ differed between sexes, I considered an average value to inform on vole detection probability and movement in a given month, since our objective was to know if these variables could affect the prediction of VAS without taking into account sex-specific differences. SCR models also provided a map (raster) with the predicted distribution and mean density of common vole activity centers within the state space $(S$ ), including the trapping grid (see Figure 3a).

\subsection{Likelihood-ratio test for extra-compensatory heterogeneity}

When modelling in SCR, a potential source of heterogeneity occurs when differences in the parameter $\sigma$ (that represents the scale of movement, proportional to home range size) and go (that represent the probability of detection by a detector theoretically placed at the home range center) which are simultaneously estimated, may compensate each other. This does not affect the density estimates (Efford \& Mowat, 2014) but unreliable estimates of $g_{0}$ and $\sigma$ may be returned by the best-selected models. I, therefore, explored whether there was evidence of extra-compensatory heterogeneity for each of the 20 CMR sessions, using the two likelihood-ratio tests ("Test 1" and "Test 2") recommended by Efford \& Mowat (2014).

2.5. Vole activity signs in linear transects

To indirectly estimate vole abundance, I used VAS presence/absence data in each of the $3 \times 1.5 \mathrm{~m}\left(4.5 \mathrm{~m}^{2}\right)$ consecutive paces/sections along the walked transect (Delattre et al., 1990). The length for the transect was $99 \mathrm{~m}$ (not reaching the end of the experimental enclosure), divided into 33 sections (like the transects currently used by ITACyL for monitoring). Within the experimental 
enclosure, I examined seven parallel transects separated by $12 \mathrm{~m}$ (Figure 1b, $2 a, b)$. Two transects were linked to field margins (1 to 7 ) and five transects were within the field (2 to 6). The total area covered for VAS assessment was $1039.5 \mathrm{~m}^{2}$ (742.5 $\mathrm{m}^{2}$ representing the alfalfa crop and $297 \mathrm{~m}^{2}$ the field margins).

Transects were surveyed for signs of activity of $M$. arvalis by the same technician (MRGB) throughout the study. In each section of each transect, the observer recorded the presence/absence of active burrows (common vole like, open burrows; BP) and of the following VAS: (i) recent soil removal (BU, burrowing), (ii) fresh droppings (DR, dropping), or (iii), presence of freshly cut vegetation accumulated at a burrow entrance (CL, clipping). Several VAS indices were calculated for each transect as the proportion of sampling units that were positive for a given VAS or combination of VAS (e.g. burrowing and/or dropping; burrowing and/or clipping; burrows and/or droppings and/or clippings, see Table 2). In order to obtain a VAS estimate for each transect, it thus summed scores (presence $=1$ / absence $=0$ ) of specific signs or combinations of signs in each section and divided it by the number of examined sections $(n=33)$.

Table 2. Summary of the different variables recorded as VAS.

Description $\left(^{*}\right)$

(i) proportion of sections with burrowing

(ii) proportion of sections with fresh droppings

(iii) proportion of sections with fresh clippings

(iv) proportion of sections with burrows and/or clippings

(v) proportion of sections with burrows and/or droppings

(vi) proportion of sections with clippings and/or droppings

(vii) proportion of sections with burrows and/or clippings and/or droppings
Abbreviation (hereafter)

\section{BU}

DR

CL

BU_CL

BU_DR

CL_DR

BU_CL_DR

$\left(^{*}\right)$ only considered if linked to common vole burrow openings (BP) 
With the standard current monitoring method used for the large-scale program of the regional government (ITACyL), two transects are conducted within field, $20 \mathrm{~m}$ away from the margin and each separated by $20 \mathrm{~m}$ (equivalent to transects 3 and 5 here) and one transect is conducted in a linear feature adjacent to the field (road ditches, weed strips, field margins; equivalent to transects 1 or 7 in our study). The calculated VAS index is the $\%$ of sections with detected burrows, droppings and clippings (equivalent to BU_CL_DR, here). 
a.

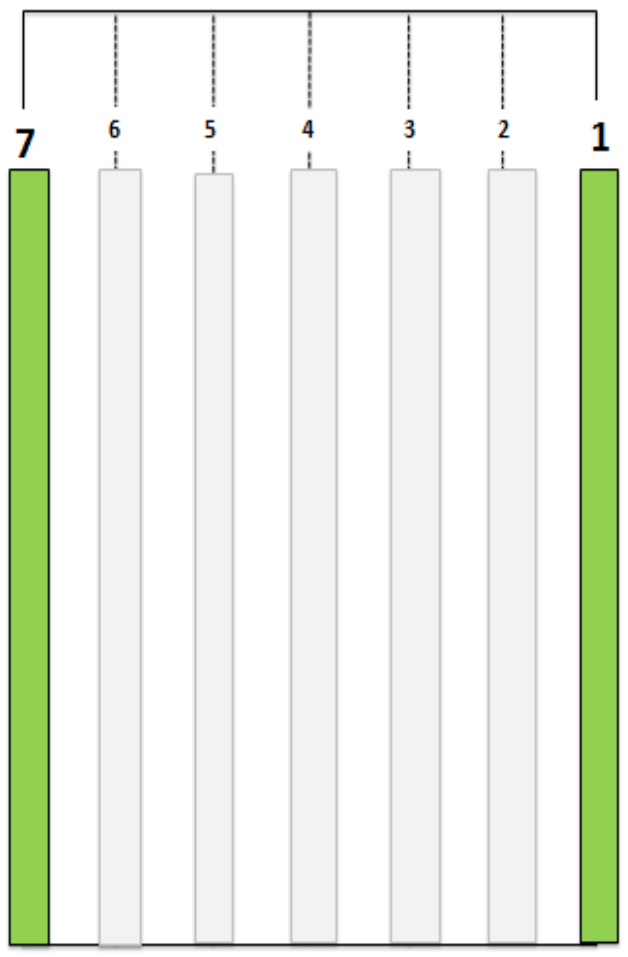

b.

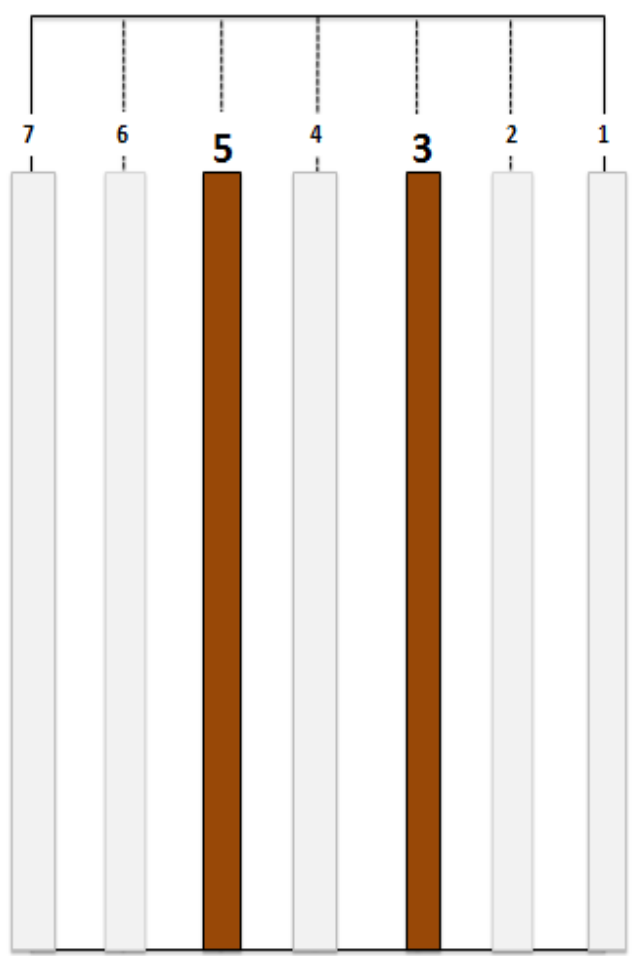

Figure 2. Location of the VAS transects located in the field margins (a, transects 1 and 7 ) or inside the field (b, transects 3 and 5 ) that most closely correspond to the standard current design used by the regional government (ITACyL) for the large-scale monitoring of vole populations.

2.6. Calculating vole densities within transect bands

Density maps of vole's activity centers (see Figure 3a) from SCR models were used to calculate density values at two different scales: (i) field scale, maintaining the original experimental enclosure size (common voles per ha) and (ii) transect scale, using only the area covered by the linear transects (voles per transect), in order to compare the VAS index with vole abundances estimated using CMR in the same transect bands (higher density variation within the 
transects bands). For the latter, I extracted vole density within each transect band using the density map produced each month (Figure 1a) and QGIS (Quantum GIS 2.18 Development (2016). I created points with coordinates in meters that corresponded with the start and end points of each transect band. I then generated a Shapefile (Layer>lines) that joined these points (Settings > snapping tolerance), and a buffer (Vector > Geoprocessing tools > Fixed distance buffer $>$ Distance $=0.75$ ) to create a Shapefile that contained the whole transect band (Figure 3b). Finally, I used the "Zonal statistics" option overlapping the raster layer that contains the vole density estimates with the Shapefile that contains all the linear transects, in order to obtain vole densities for each linear transect. 
a.

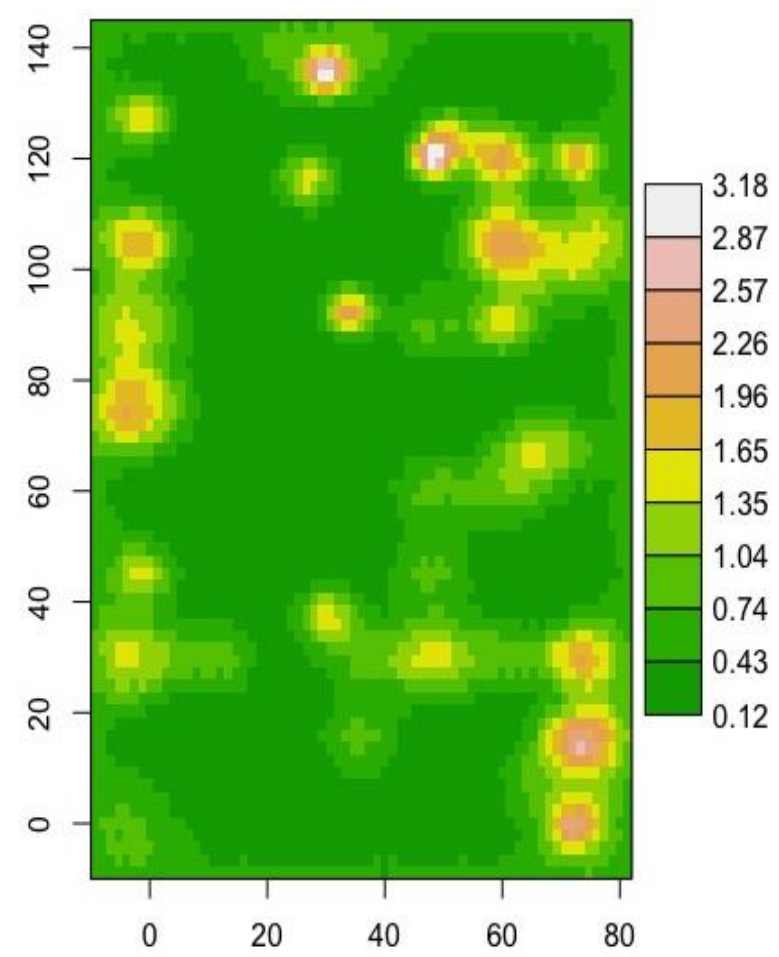

b.

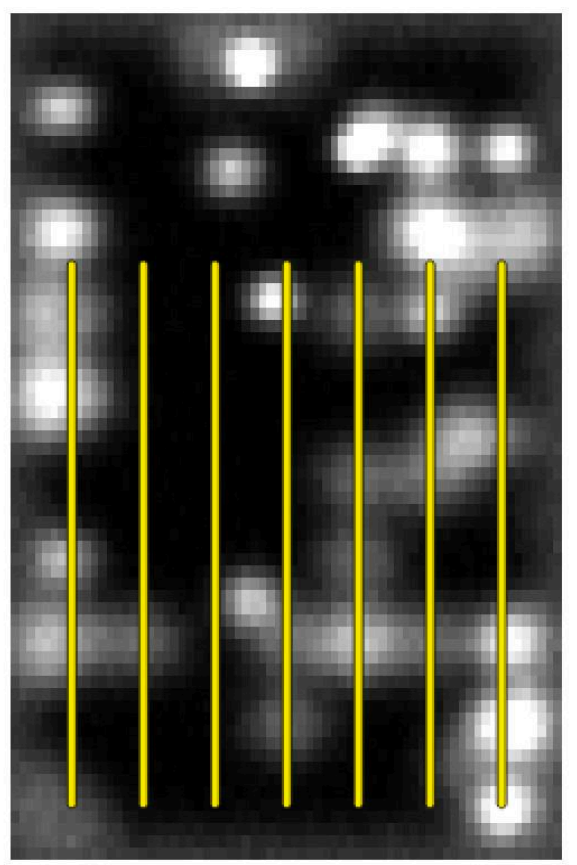

Figure 3. Example of density map obtained as a SCR model output (raster) showing the mean density (voles per $100 \mathrm{~m}^{2}$ ) of vole activity centers within the state space $S(\mathrm{a})$. This model output is for March 2017. The state-space region covers $25740 \mathrm{~m}^{2}$, each pixel is of $1.45 \mathrm{~m}^{2}$ and the scale is in individuals $/ 100 \mathrm{~m}^{2}$. The trap array covered 9720 $\mathrm{m}^{2}(72 \times 135 \mathrm{~m})$. Distance units on the $X$ and $Y$ axes are in meters. The spatial analysis (QGIS) of the raw maps obtained in the SCR model outputs: (b) field scale (different scale of grey colors but the same map), maintaining the original size of the experimental enclosure (number of voles per ha) and the transect scale (yellow line transects), using only the area covered by the transects (number of voles per linear transect).

\subsection{Statistical analysis}

Associations between density and VAS were tested using Generalized Linear Models (GLMs), using a log-transformation of vole density (log-D) to obtain 
linear relationships with VAS. GLMs allowed comparisons between the predictive value of the different combination of VAS (comparing the proportion of variance explained).

To explore an additional influence of month on the association between density and VAS, generalized additive models (GAMs) were used with a smoothing function (thin-plate spline) on month. This choice was made following initial explorations which revealed that the effect of month was strongly nonlinear. Season was later simplified by regrouping months into a factor based on the GAMs model outputs.

Initial explorations revealed that both the influence of vegetation height and the covariate $\left(g_{0}\right)$ of vole behavior were linear (and were therefore fit linear models using GLMs) but not for the influence of the covariate $(\sigma)$ of vole behavior, that was non-linear (and was thus fitted using GAMs). In order to understand which variables contributed to an observed seasonal effect in the association of density and VAS, I used two separate models that included (i) vegetation height or (ii) vole behavior parameters $\left(g_{0}\right.$ and $\sigma$, derived from monthly SCR models) as explanatory variables. R (v3.1.3) was used for all statistical analyses (R Core Team, 2015). GAMs were built using the R package “mgvc" V 1.8-25 (Wood, 2018). 


\section{Results}

3.1. SCR Models outputs and tests for extra-compensatory heterogeneity

SCR models were run for a total of 20 monthly CMR sessions (Table 3). For all but two months, I found no evidence of extra-compensatory heterogeneity (Table 4). For January 2017 and April 2017, however, statistical evidence for extra-compensatory heterogeneity was found (Test 2 value $<0.001$; Table 4). In July 2017, Test 2 was also significant but differences in density between the Null model and the most parsimonious model indicate that extra-compensatory heterogeneity was not an issue for this particular month. Hence, for two months, the parameter estimates $g_{0}$ and $\sigma$ should be viewed with a lack of confidence, although the estimation of density was correct. Consequently, all density estimates (20 months) were used when analyzing associations between density and VAS but only a subset of 18 months (excluding January and April 2017) were used when incorporating go and $\sigma$ or when including these parameters as explanatory variables. The latter analyses were repeated using all the data gathered in the field (CMR sessions) in order to check that both sets of data (with 18 vs 20 monthly density comparisons) gave similar results (reported in Appendix 2 - S1 Supporting Information).

Throughout the study, the movement parameter $\sigma$ varied with maximum values of $15.46 \pm 2.91$ and minimum values of $5.52 \pm 0.43$ (Table 3). The basal detection probability parameter, go, also varied with maximum values of $0.32 \pm$ 0.04 and minimum values of $0.05 \pm 0.01$ (Table 3 ). 


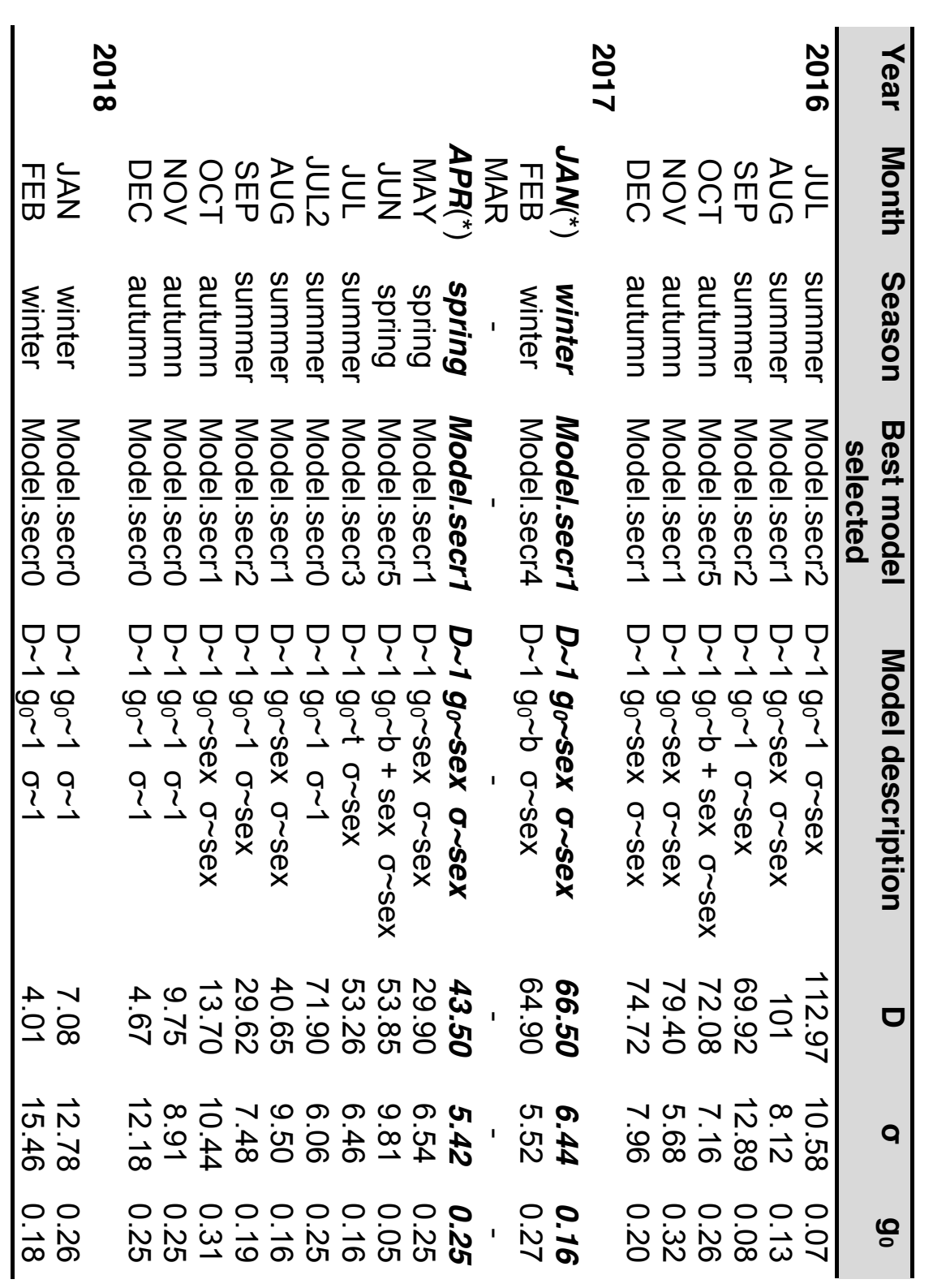

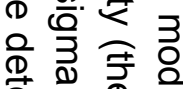

市. 弆

흘 웡 웅

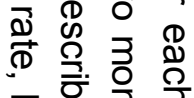

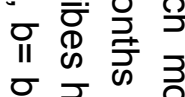

市 ত ‥ 응 응 䨖 $\mathbb{D}$

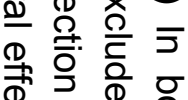
욱음 응 으음 궁 을 票文离 응 유유 ᄚ

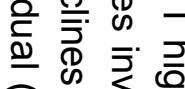
त) فำ

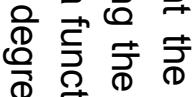
造 웅요 익

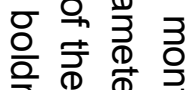
造 的志 कू की व

윽 혹은 은

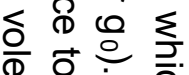

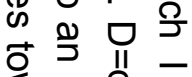

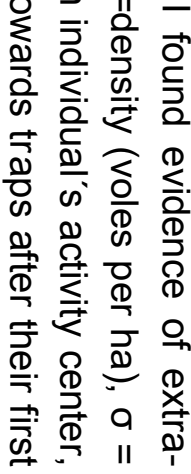


Table 4. Tests for heterogeneity in detection parameters estimated by fitting a twoclass finite mixture. 'Test 1" and "Test 2" are probability values for likelihood-ratio tests (variation in $\sigma$ given compensatory variation in $\mathrm{g}_{0}$, and extra-compensatory variation in $g_{0}$ ). The table also reports the density derived from SCR models without covariate influence on $\mathrm{g}_{0}$ or $\sigma$ (Null model) or derived from the best-selected models (Table 3; see also Chapter 1) that allowed for an effect of behavior, time or sex on $g_{0}$ or an effect of sex on $\sigma$.

\begin{tabular}{|c|c|c|c|c|c|}
\hline \multirow[b]{2}{*}{ Year } & \multirow[b]{2}{*}{ Month } & \multirow[b]{2}{*}{ Test 1} & \multirow[b]{2}{*}{ Test 2} & \multicolumn{2}{|c|}{ Density (CMR) } \\
\hline & & & & $\begin{array}{c}\text { Best } \\
\text { selected model }\end{array}$ & Null model \\
\hline \multirow[t]{6}{*}{2016} & JUL & 0.301 & 0.90 & 112.97 & 110.96 \\
\hline & AUG & 0.005 & 0.05 & 101 & 100 \\
\hline & SEP & 0.019 & 0.90 & 62.28 & 62.60 \\
\hline & OCT & 0.001 & 0.02 & 69.18 & 71.88 \\
\hline & NOV & 0.000 & 0.01 & 79.03 & 77.20 \\
\hline & DEC & 0.000 & 0.01 & 75.99 & 73.85 \\
\hline \multicolumn{6}{|l|}{2017} \\
\hline & $\boldsymbol{J A N}\left({ }^{*}\right)$ & 0.000 & $<0.001$ & 65.14 & 64.19 \\
\hline & FEB & 0.000 & 0.24 & 66.07 & 69.30 \\
\hline & $\operatorname{APR}\left({ }^{*}\right)$ & 0.000 & $<0.001$ & 42.26 & 41.47 \\
\hline & MAY & 0.001 & 0.01 & 29.06 & 26.92 \\
\hline & JUN & 0.001 & 0.12 & 53.85 & 40.31 \\
\hline & JUL & 0.000 & $<0.001$ & 53.26 & 47.62 \\
\hline & JUL2 & 0.470 & 0.94 & 71.9 & 71.9 \\
\hline & AUG & 0.000 & 0.04 & 40.65 & 38.58 \\
\hline & SEP & 0.210 & 0.67 & 29.60 & 29.11 \\
\hline & OCT & 0.020 & 0.02 & 13.70 & 13.79 \\
\hline & NOV & 0.370 & 0.17 & 9.75 & 9.75 \\
\hline & DEC & 0.372 & 0.17 & 4.67 & 4.67 \\
\hline \multicolumn{6}{|l|}{2018} \\
\hline & JAN & 0.096 & 0.13 & 7.08 & 7.08 \\
\hline & FEB & 0.012 & 0.67 & 4.01 & 4.01 \\
\hline
\end{tabular}

$\left({ }^{*}\right)$ In bold and italic, the months that were excluded for the analysis because of extracompensatory heterogeneity.

3.2. Associations between VAS indices and vole density at field level During 2016-2018, common vole abundance varied 28-fold, with a minimum abundance (estimate \pm SD) of $4.0 \pm 1.4$ voles/ha (February 2018) and a maximum of $112.97 \pm 24.5$ voles/ha in July 2016 (Figure 1a). Over the same period, the VAS index varied 8-fold, from a minimum of 0.06 to a maximum of 0.49 (Figure1b). 


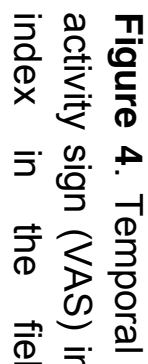

으스요.

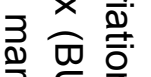

鱼 $\subset$ क

?

흠ํํㄹ

우슈ํㅇ

बे

윰

+응 을

@i 응

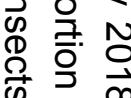

요

$\rightarrow$ 于

ข

ํㅡ હ

ㄴㅇㅡ 콬

훙으

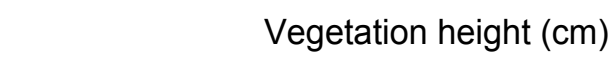

Voles per $\mathrm{Ha}$

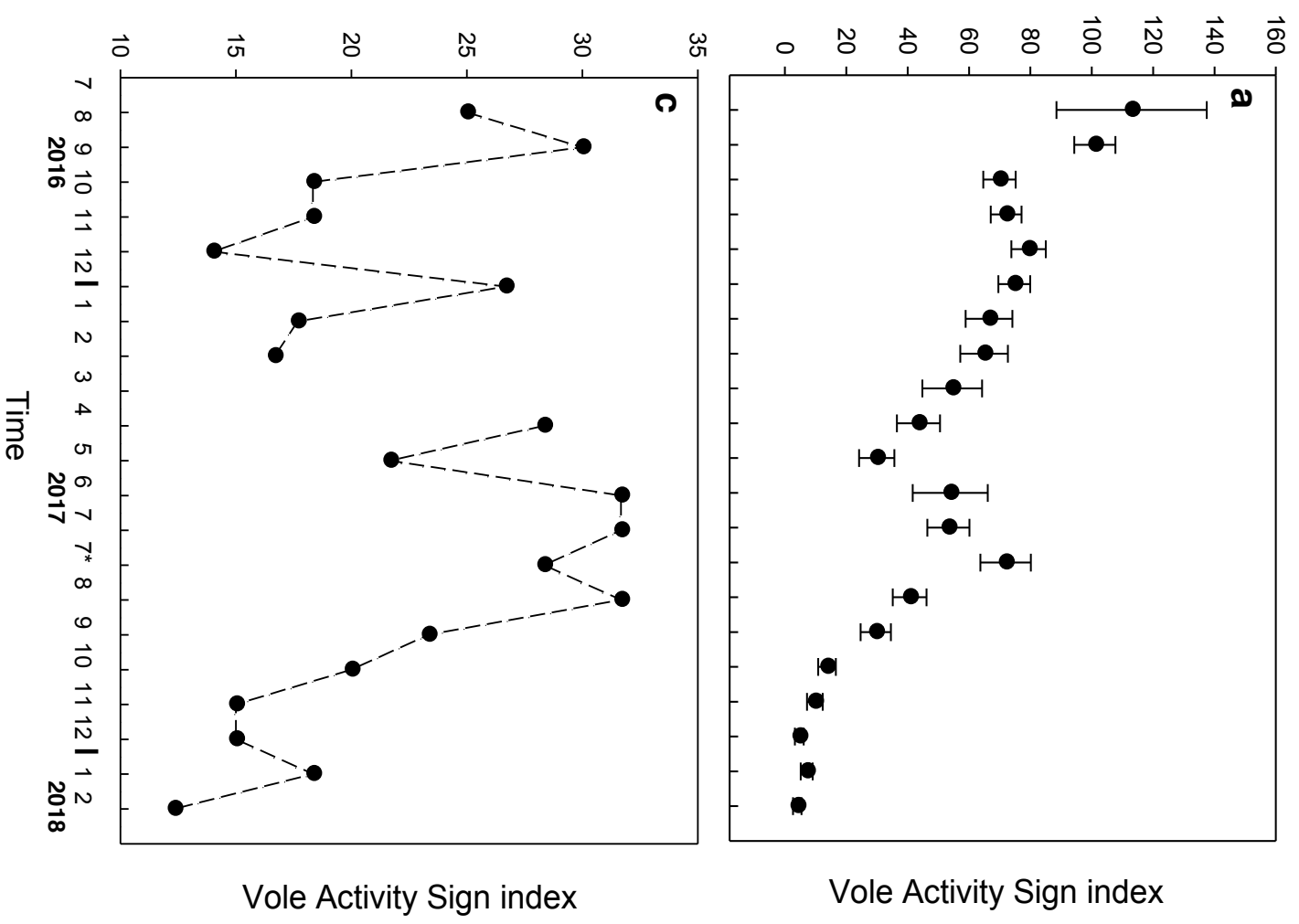

ำ 응

节

농

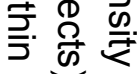

ज्ञ क

요 $\mathbb{\mathbb { D }}$

$\overrightarrow{\bar{\Phi}} \stackrel{0}{0}$

을 옴

후 $\frac{0}{\overrightarrow{0}}$

市.

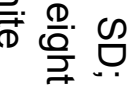

응

क्ष

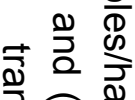

กิ

क ळ

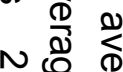
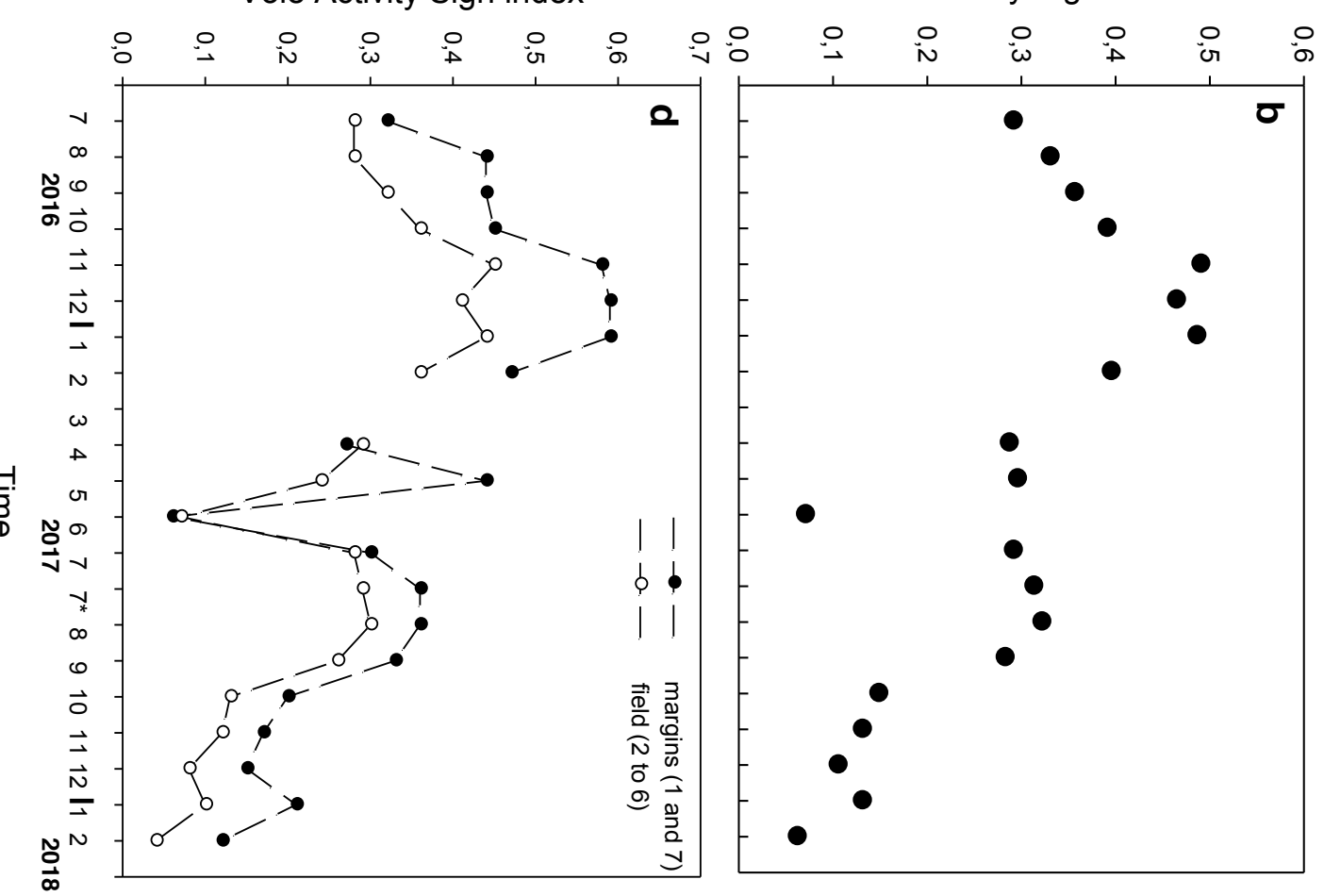
Following SCR modelling to derive densities per ha, these vole densities were modelled against an average VAS index (average from the 7 transects) with the average based on either a single type of VAS (e.g. fresh droppings) or different combinations of VAS (see Table 2) to identify the best predictors (Table 5). Additional models repeated the analysis but only used the transects inside the alfalfa filed (2 to 6 ) therefore excluding those of the field margins (1 and 7). Fresh clippings alone were a poor predictor while fresh droppings and burrowing were better at predicting vole density. However, the two best predictors of estimated vole densities were based on combinations of VAS (BU_DR or BU_CL_DR; Table 5). The index used by the regional government ITACyL, which is a combination of three types of vole activity signs

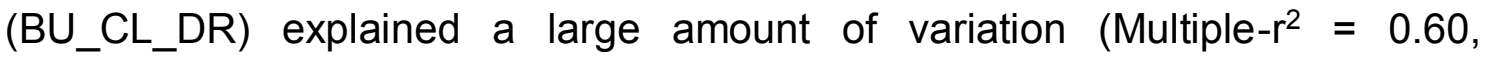
Adjusted- ${ }^{2}=0.57$ ), with the trend being significantly different from a slope of 0 $\left(F_{1,18}=27.08, p<.001\right)$. As such, the index is an accurate predictor of vole density when using an averaged value from the 7 transects. The average for the transects 2 to 6 had a slightly better precision (see Table 5; Figure 5). 


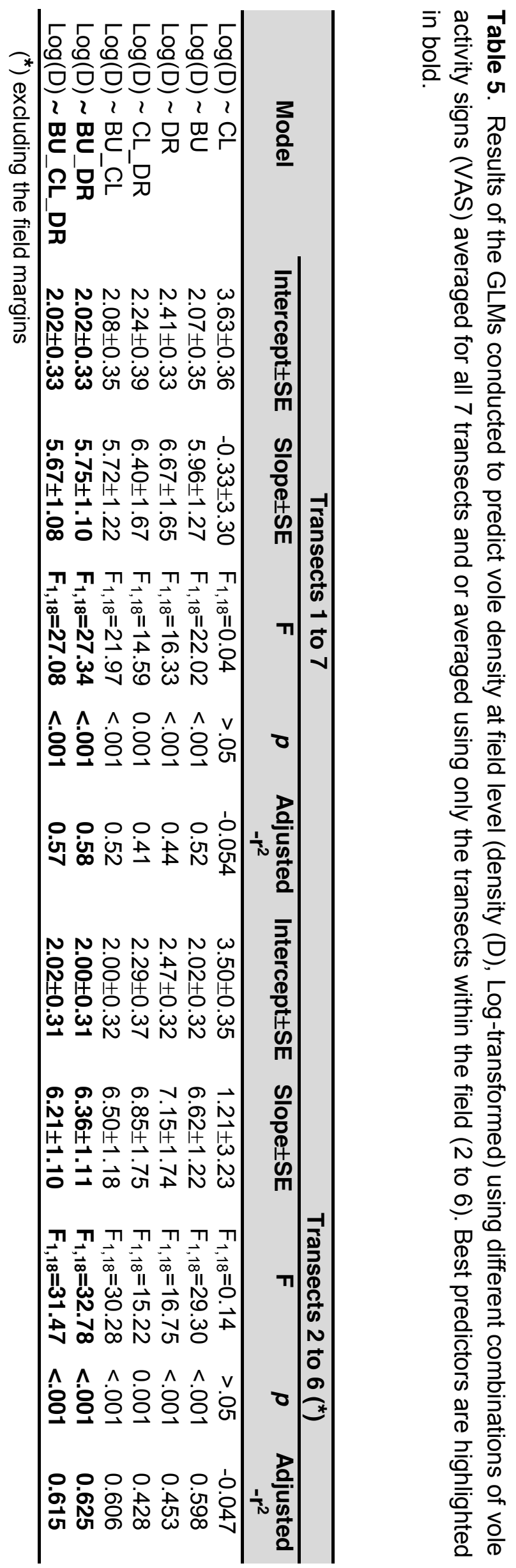




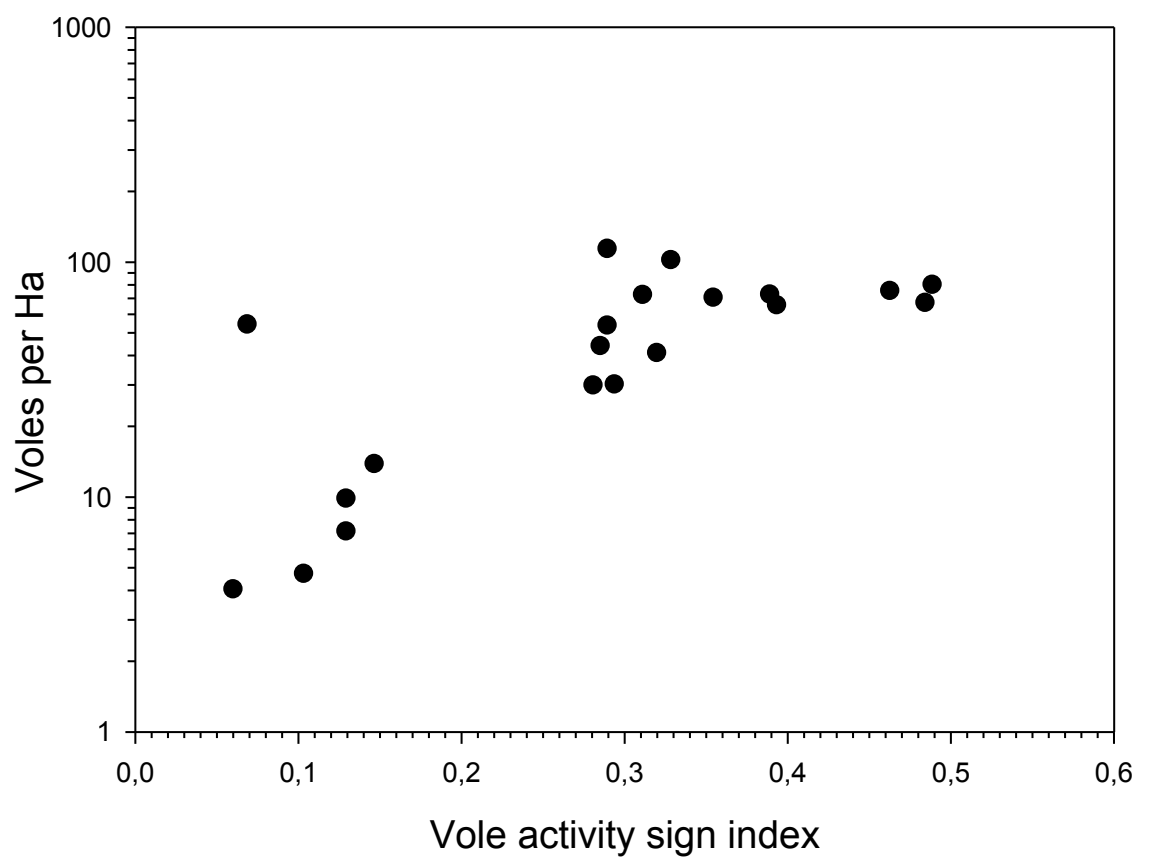

Figure 5. Relationship between common vole density (voles per ha, log-scale) at field level obtained from CMR and SCR modeling and the VAS index used by regional government, ITACyL (BU_CL_DR).

3.3. Factors affecting the relationship between VAS and field level vole density

\subsubsection{Seasonal effects}

Although VAS (BU_CL_DR) explained a large amount of variance in relation to density, there was still a relatively large proportion of unexplained variation (c. $40 \%$ ). It was suspected that season may have influenced some of the variance. To test for this, the average VAS for all transects (1-7) was used in subsequent analysis. Adding a smoothed influence of Month improved the model predictions (Table 6), with $r^{2}$ values reaching 0.80 for the two best VAS predictors (BU_DR and BU_CL_DR). 
Table 6. Results of the GAM models testing for an additive influence of Month on the association between vole density (Log (D)) and each VAS.

\begin{tabular}{|c|c|c|c|c|}
\hline Model & edf & $\mathbf{F}$ & $p$ & Adjusted-r $r^{2}$ \\
\hline $\log (\mathrm{D}) \sim \mathrm{CL}+\mathrm{s}(\mathrm{MONTH})$ & 1.88 & $\mathrm{~F}=1.48$ & $>.05$ & 0.07 \\
\hline $\log (\mathrm{D}) \sim \mathrm{DR}+\mathrm{s}(\mathrm{MONTH})$ & 1.72 & $F=1.34$ & $>.05$ & 0.50 \\
\hline $\log (\mathrm{D}) \sim \mathrm{BU}+\mathrm{s}(\mathrm{MONTH})$ & 2.59 & $F=6.50$ & 0.003 & 0.78 \\
\hline Log(D) DR_CL+s (MONTH) & 1.83 & $F=1.41$ & $>.05$ & 0.48 \\
\hline $\log (\mathrm{D}) \sim \mathrm{BU} \_\mathrm{CL}+\mathrm{s}(\mathrm{MONTH})$ & 2.47 & $F=6.01$ & 0.05 & 0.76 \\
\hline Log(D) BU_DR+s (MONTH) & 2.54 & $F=6.54$ & 0.003 & 0.80 \\
\hline Log(D) BU_CL_DR+s (MONTH) & 2.55 & $F=6.45$ & 0.004 & 0.80 \\
\hline
\end{tabular}

The relationships with month was non-linear with the effective degrees of freedom validating the use of a smoothing term (Table 6). The predictive power of the best VAS index (BU_CL_DR) was greatly improved by adding a smoothed monthly effect (deviance explained $=84.20 \%$ ). The same was observed for the BU_DR index (deviance explained $=84.40 \%$ ).

A cautionary note should be included whereby a trend was noted in the residuals regarding month. The association between log (density) and VAS (Figure 6) showed a departure in log (density) and VAS, meaning that fewer VAS were detected than would be expected given the density during springsummer. 


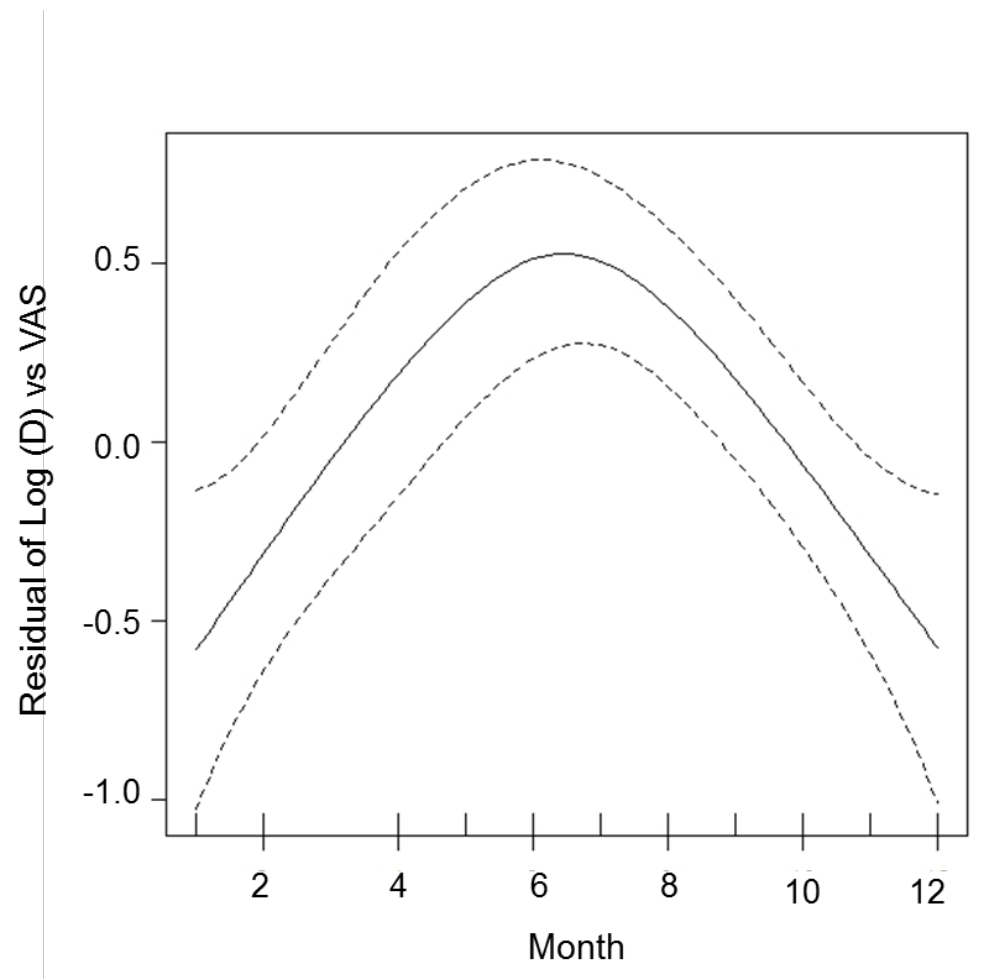

Figure 6. GAM residuals showing an underestimation in some winter months, and an overestimation in summer months between log (density) and VAS (BU_CL_DR).

In order to simplify this seasonal effect on VAS relative to density, I created a categorical variable, "season", which aggregated months into springsummer months (S-S, April to August) and autumn-winter months (A-W, September to March). This simplified our model while maintaining a comparable $r^{2}$ value $\left(r^{2}=0.78\right)$. Doing so allowed the calculation of density estimates from VAS for each period separately (see equations (1) and (2) below). I could not test for a season and VAS interaction due to sample size limitations.

$$
\begin{array}{ll}
\text { Autumn-winter (A-W): } & D=\exp (V A S * 5.62+1.62) \\
\text { Spring-summer (S-S): } & D=\exp (V A S * 5.62+2.44)
\end{array}
$$


3.4. Variables contributing to monthly variation in the VAS performance

Additional variables which could explain the residual patterns observed VAS when predicting vole density according to month were tested. I first considered changes in vegetation height, and secondly changes in vole behaviour. For these analyses, I only considered the most complete VAS index (BU_CL_DR) as a dependent variable.

Vegetation height varied among months, with plants being taller in months during the $S-S$ season (average of $32 \mathrm{~cm}$ ) than in months during A-W (12 cm; Figure 1c; Figure 7).

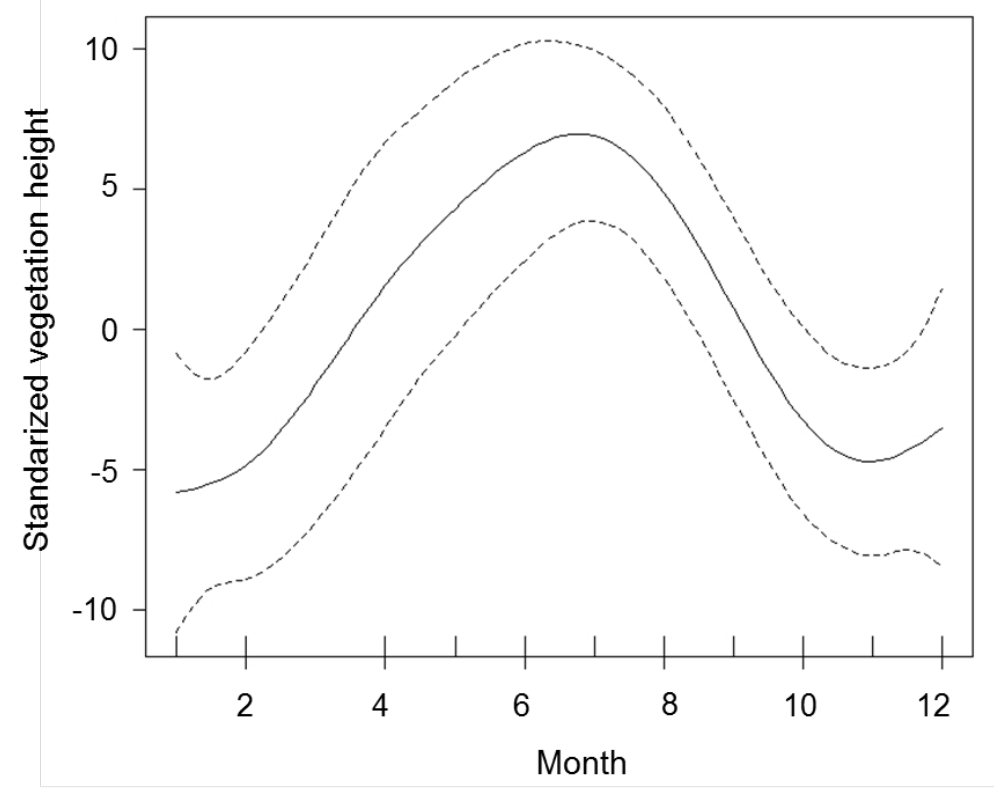

Figure 7. GAM model output showing the monthly variations in vegetation height. 
VAS was found to be lower than would be expected given vole density when vegetation in the field was taller, (BU_CL_DR Log (D) + VEGETATION; $F_{2,17}=22.20 ; p<.001 ;$ Multiple- $r^{2}=0.72 ;$ Adjusted $-r^{2}=0.69 ;$ Figure 8) .

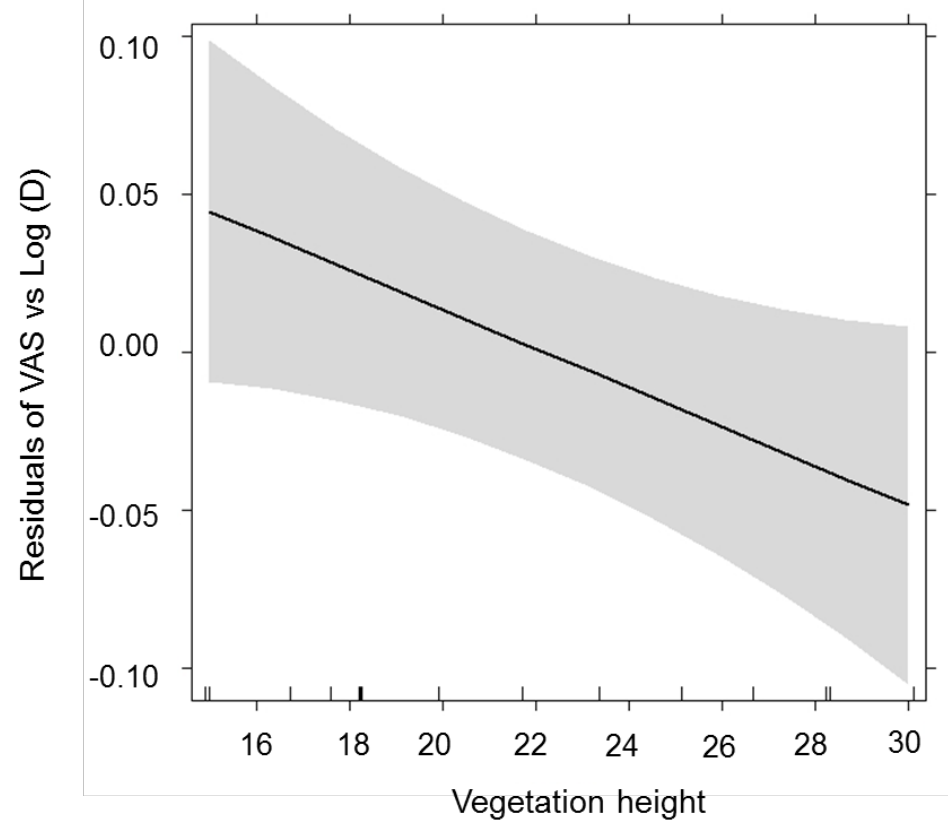

Figure 8. LM output showing the variations in the residuals of the association between VAS (BU_CL_DR) and Log (D).

To explore an influence of vole behavior, the $g_{0}$ and $\sigma$ parameters obtained from the SCR models were included in subsequent models. $g_{0}$ is related to the probability of detecting a vole and $\sigma$ represents vole movement (Table 7). Greater values of $g_{0}$ indicated that voles were more detectable by trapping, whereas greater values of $\sigma$ indicated that voles moved further away from their activity center (greater home ranges).

I found that the precision of VAS for predicting vole density decreased with increasing movement away from activity centers (Figure 9) and increased with increasing likelihood of detection $g_{0}\left(B U \_C L \_D R \sim \log (D)+\log \left(g_{0}\right) ; F_{2,15}\right.$ 
$=38.87 ; p<.001 ;$ Multiple- $r^{2}=0.83 ;$ Adjusted $-r^{2}=0.81$ in a linear way (Figure 10).

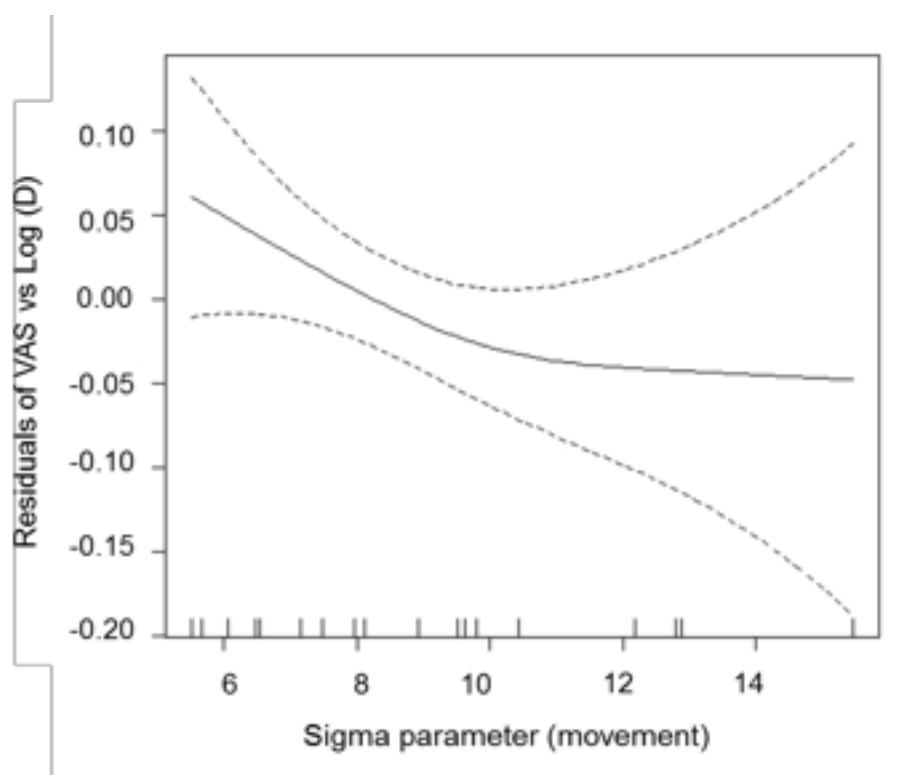

Figure 9. Predicted relationship between VAS (BU_CL_DR) relative to vole density and to the parameter $\sigma$ (movements).

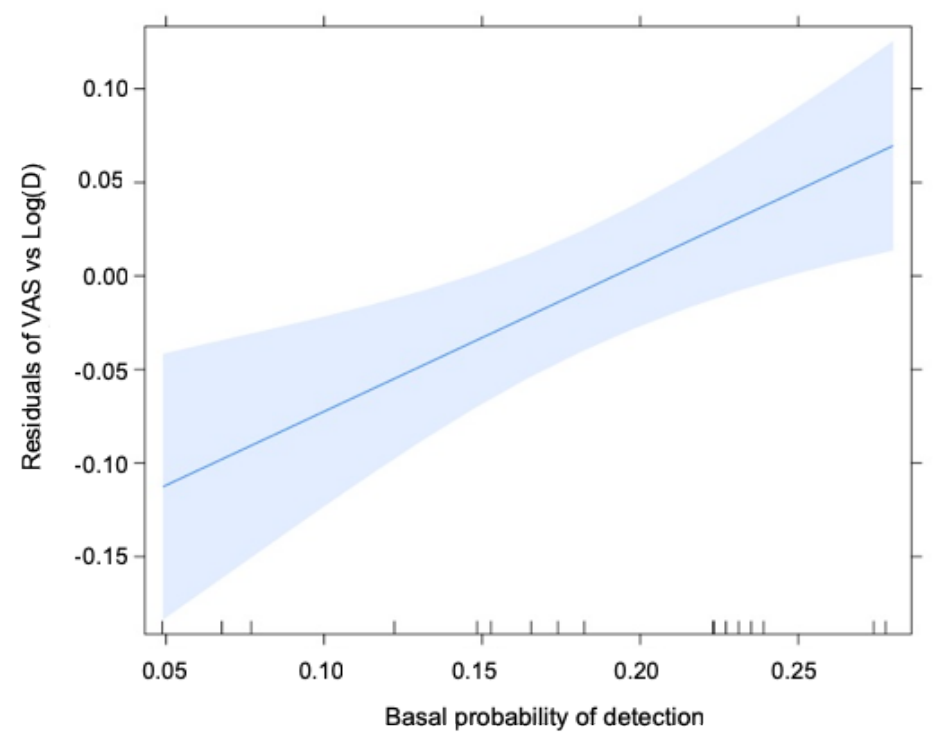

Figure 10. Residuals of the relationship between VAS (BU_CL_DR) relative to vole density and to the parameter $g_{0}$ (basal probability of detection). 
3.5. Associations between VAS indices and vole density at transect level

The predictive ability of the full VAS index (BU_CL_DR) was compared to vole densities extracted from transect locations. Transects used for comparison were either from field margins (transects 1 and 7 , see Figure 2) or within field (transects 3 and 5, see Figure 2). The GAM models also included a seasonal effect (smoothing of the Month effect: Log (D) BU_CL_DR + s (MONTH)).

At the transect level, estimated vole densities ranged from 0 to 472 voles per ha, and their associated VAS ranged from 0 to 0.84 (Figure 11). The model was better able to predict vole densities when transects were inside the field (adjusted $r^{2}=0.60$; deviance explained $=64.8 \%$ ) than in the field margins (adjusted $r^{2}=0.27$; deviance explained $=37.5 \%$ ). Moreover, the seasonal effect on the density to VAS relationship was not significant for transects in field margins $(s(M O N T H)):$ edf $=4.24 ; \quad F=1.71 ; p=0.157)$ but the effect was significantly different to 0 for transects inside the field (edf=3.58; $F=5.81$; $p=0.007$ ) showing that month has an important role in density-VAS relationship. Overall, predictions of local, transect level, vole density from VAS were thus better for transects located inside the field than in the field margins. Average vegetation height (Min-Max) in the margins ranged from 17.5 to $60 \mathrm{~cm}(26.5 \mathrm{~cm}$ during Autumn-Winter, and $47 \mathrm{~cm}$ during Spring-Summer). 


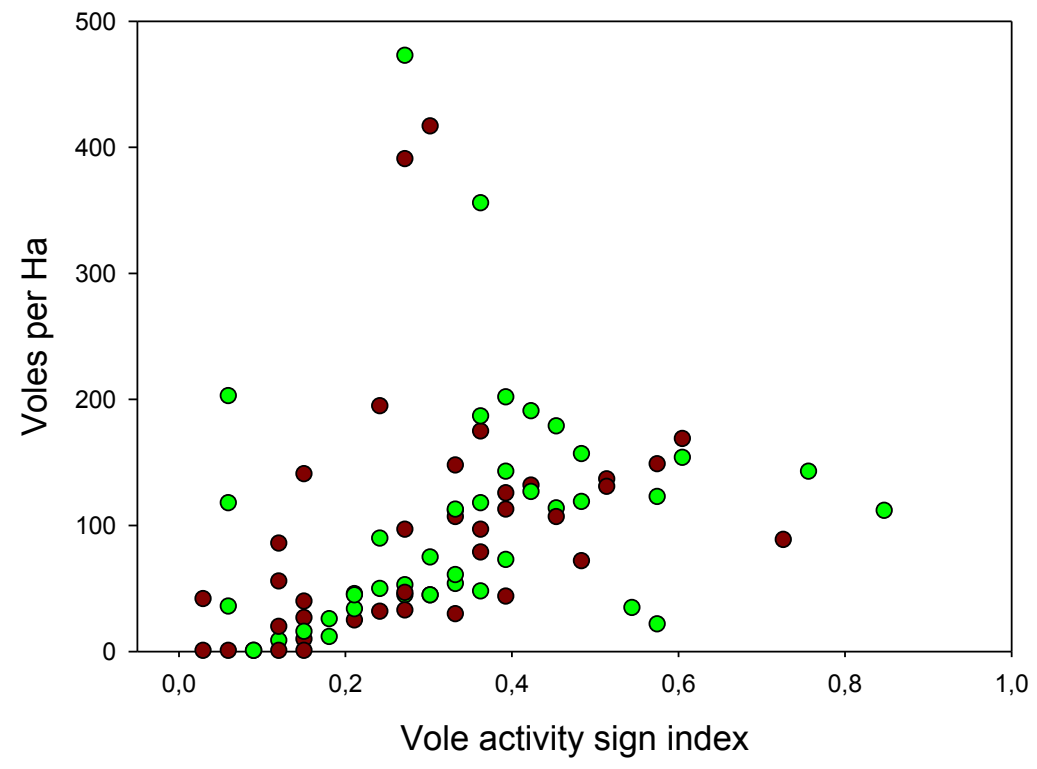

Figure 11. Relationship between common vole density (voles/ha, Log scale) at transect level obtained from CMR and SCR modeling and the VAS index (BU_CL_DR). Colored dots denoted the different position of the linear transect bands (green dots = transects in the margins ( 1 and 7$)$ and brown dots = transects within the plot ( 3 and 5$)$ ). 


\section{Discussion}

In the present study, I compared a VAS index with vole population density estimated through SCR modelling, the method considered the most precise and least biased to date (Fauteux et al., 2018; Gerber \& Parmenter, 2015; Krebs et al., 2011; Parmenter et al., 2003; Romairone et al., 2018). I showed that: i) VAS index measured in transects within an alfalfa field allows vole density to be accurately predicted up to 113 vole per ha (the maximum recorded in the experimental field during the study), and ii) VAS measured inside the field provided better predictions than VAS measured in field margins. It was also found that residual error between vole density and VAS varied seasonally, with fewer VAS detected than expected from density during spring-summer as compared to autumn-winter. This seasonal variation was putatively explained in part by a lower detectability of VAS with increasing vegetation height, and in part by changes in vole behavior, with a lower VAS detectability when vole detectability in traps (an SCR-index of vole activity) was also reduced.

4.1. Relationship between VAS and vole density at field level

I found that some indirect indices used in isolation were poor predictors of density, like clippings, while others provided better predictions, such as droppings and burrowing. Indices based on burrows have been suggested as good indicators of vole activity (Liro, 1974; Steiner et al., 2007; Jareño et al., 2014), although Gervais (2010) found that simply counting them was likely to be misleading. The best predictors of density at field level were based on combination of VAS (BU_DR or BU_CL_DR). The main index used by the 
regional governmental agency, ITACyL, is a combination of the three types of VAS (BU_CL_DR) and was confirmed as a good predictor of vole density at field level, up to 113 voles per ha (Figure 5). However, its performance was poorer for predicting vole density at transect level (in this case, with local densities up to 472 voles per transect), and even poorer for assessments done in field margins as compared with inside field (Figure 11). Because vole densities in farmlands can be much higher during outbreaks $(\sim 1000$ vole/ha in habitats such alfalfa fields; Vidal et al., 2009; Jacob \& Tkadlec, 2010; Delibes, 1989) or even 2000 vole/ha (Bryja et al., 2005), it would be necessary to further calibrate the VAS index at higher vole densities than those recorded in this study.

The results of this study are in agreement with those of Jareño et al., (2014), which showed that VAS predicted vole abundance estimates based on simpler trapping methods. However, in the Jareño et al., (2014) study, the density estimates were less precise because of the method used to calculate them (in terms of trapping design and statistical methods, e.g. Schnabel method, non-spatial; see Introduction in Chapter 1, e.g. main limitations of traditional CR models) and the temporal spread of the study did not allow them to assess seasonal variations in the relationship, which has been shown here to be an important part of the relationship between VAS and density. Regarding the spatial processes, SCR modelling makes use of the spatial locations of the encountered individual (Efford, 2004; Royle \& Young, 2008; Borchers \& Efford 2008; Gardner et al., 2009), and therefore provides more accurate estimates of population densities. However, some specific requirements must be taken into account when applying this spatial methodology (e.g. effort employed such as 
number of trapping stations, number of traps or adjust the trap configuration the minimum distance between traps- to the movement of the species study; see Chapter 1; Romairone et al., 2018). In the present study, the sampling effort was above the minimum requirement (Fauteux et al., 2018), and thus, this should not have been an issue for the VAS index validation.

Amongst the VAS indices considered in the study by Jareño et al., (2014), the presence of fresh clippings and/or droppings were the best predictors. Here I have found that the clipping index was the poorest predictor, while droppings and burrows provided better predictions. Regarding the clippings index, the results are in concordance with the study of Gervais (2010), which suggested that higher rates of vegetation clipping may occur at the beginning of the season, when voles appear to store piles of fresh clippings, but that latterly comparisons cannot be made through time, but only through space at the same time. In any case, the combination of indices appeared as the best option to predict vole density.

\subsection{Variables affecting the relationship between VAS and vole density}

A main limitation of the VAS method is that it lacks the precision of trapping methods and may be subject to biases. Some potential sources of bias have been explored within this analysis as well as the means of how to take them into account in order to improve predictions. A bias can arise when discrepancies appear between different observers. In this study, the VAS methodology was carried out by the same technician for the entire study period, therefore eliminating among-observer differences. However, when using VAS at large scale and over time, it would be important to ensure that a sufficiently large 
number of well-trained observers are used, or that they are relocated among different areas to avoid potential temporal or spatial biases in estimates arising from observer differences.

I found that some of the unexplained variation in the relationship between density and VAS was due to a seasonal effect. For a given vole density, VAS was lower in spring-summer than in autumn-winter. This finding allows for improves density estimates from VAS by regrouping the spring-summer months (S-S, April to August) and the autumn-winter months (A-W, September to March) in order to calculate density estimates (with two different equations) from VAS for each period separately.

This seasonal variation in VAS performance was partly due to differences in vegetation height and changes in the behavior of voles (most markedly in $g_{0}$ - basal probability of detection). VAS relative to density decreased with vegetation height, meaning that I detected relatively fewer VAS than expected from density when the field vegetation was taller. This may be because VAS becomes only partially visible to the observer, or with more vegetation cover voles do not need to move much for feeding, leading to less activity that can be detected via VAS. This interpretation is in line with results reported which have shown that with a reduction in vegetation cover, small mammals are exposed to potential predation (Preston, 1990), which leads to behavioral changes in common voles (Jacob \& Brown, 2000). Therefore, it would be important that either searching effort increases with vegetation height or cover, or that vegetation height and cover are systematically noted when carrying out monitoring based on VAS to adjust predictions taking this into account. 
Regarding vole behavior, the results have shown that more VAS was detected than expected from density when voles were more detectable in traps (greater go). Regarding capturability, the results may be related to the relative above-ground vs below-ground activity. At certain times of the year, voles may spend more time above ground for different activities such as foraging or mating (Brown, 1988; Ylönen \& Ronkainen, 1994; Schmitz et al., 1997; Brown et al., 1999; Lima \& Bednekoff, 1999), subsequently leaving more signs and having a greater probability of being trapped. Conversely, at other times activity may be concentrated in burrows, for instance reducing locomotory activities under high predation risk (Boworski \& Owadowska, 2009). Other factors that vary seasonally could also explain variation in VAS predictive performance and could be explained in future studies, for instance variations in rainfall, as rain could wash out some of the remains left by voles (droppings, clippings).

\subsection{Differences in VAS in margins versus field}

The performance of the most complete VAS index was compared considering transects in field margins ( 1 and 7 ) or within field (3 and 5) with vole densities estimated within linear transect bands (range 0 to 472 voles per ha), including a seasonal effect. Predictions of vole densities were more precise when VAS was carried out within the field than those in the field margins. In a previous study, Rodríguez-Pastor et al., (2016) showed that field margins represent a key habitat for common voles in Mediterranean agricultural landscapes and can harbor an average vole abundance 2.3 times higher than inside fields. However, they also found that the use of margins by common voles was dynamic depending on crop type, season, vole abundance, and vegetation 
characteristics. In particular, differences between margin and field were least marked in alfalfas (as compared with cereal or fallow), particularly in summer. Similarly, here I found higher VAS estimates in margins that in fields in most months, consistent with previous findings. Margins are narrow habitats that voles inhabit but also use to disperse, and thus temporally harbor both resident and transient voles. At some point, a spill-over of common voles from margins towards the inside of the fields ("edge effect") occurs at maximum vole density with a subsequence spread of voles inside the fields (Rodriguez-Pastor et al., 2016). Therefore, accurately monitoring voles is critical in terms of predicting potential impacts of vole abundance on crop damage. It would be therefore advisable to confirm the results in other crops to assess the reliability of VAS when assessing vole density in margins.

Finally, this study was framed in a maximum estimate vole density up to 113 voles per ha, so it would also be desirable to study what would happen when reached more than 1000 voles per ha and also to correlate these higher vole densities with crop damages, in order to identify alarm thresholds to trigger management actions. 


\section{Conclusions}

Obtaining reliable data on vole abundance is often a methodological challenge, and there is a constant need for updating, validating and developing simpler or cheaper methods for ecological and management studies (Krebs, 1999; Tellería, 2004; Witmer, 2005). Although live-trapping (CMR) is considered the "golden standard" (Hickey \& Sollmann, 2018) compared with other indirect methods that estimate abundance based on activity signs (Jareño et al., 2014), it is labour intensive (e.g. time-consuming and requiring expertise) and costly (e.g. investment in many traps) to maintain a long-term monitoring program when working at large spatial scales. It has been shown here that (i) indices based on vole activity signs (VAS) are a reliable indirect method for estimating vole abundance and represent an important tool for large-scale monitoring of a rodent pest like the common vole, (ii) the relationship between vole density and VAS varied seasonally, with fewer VAS detected than expected from density during spring-summer as compared with autumn winter. This seasonal variation may be explained by a lower detectability of VAS with increasing vegetation height, or reduced vole detectability in traps (potentially indicative of a reduced surface activity/increased subterranean residency) and (iii) although VAS performed relatively well, predictions appeared to be less precise when used in field margins rather than within the field, at least in our experimental alfalfa field. Given the importance of measuring abundance in field margins and linear habitats, it would therefore be important to better understand these differences to improve predictions in field margins. 


\subsection{Chapter "3"}

Vole sex-ratio assessment

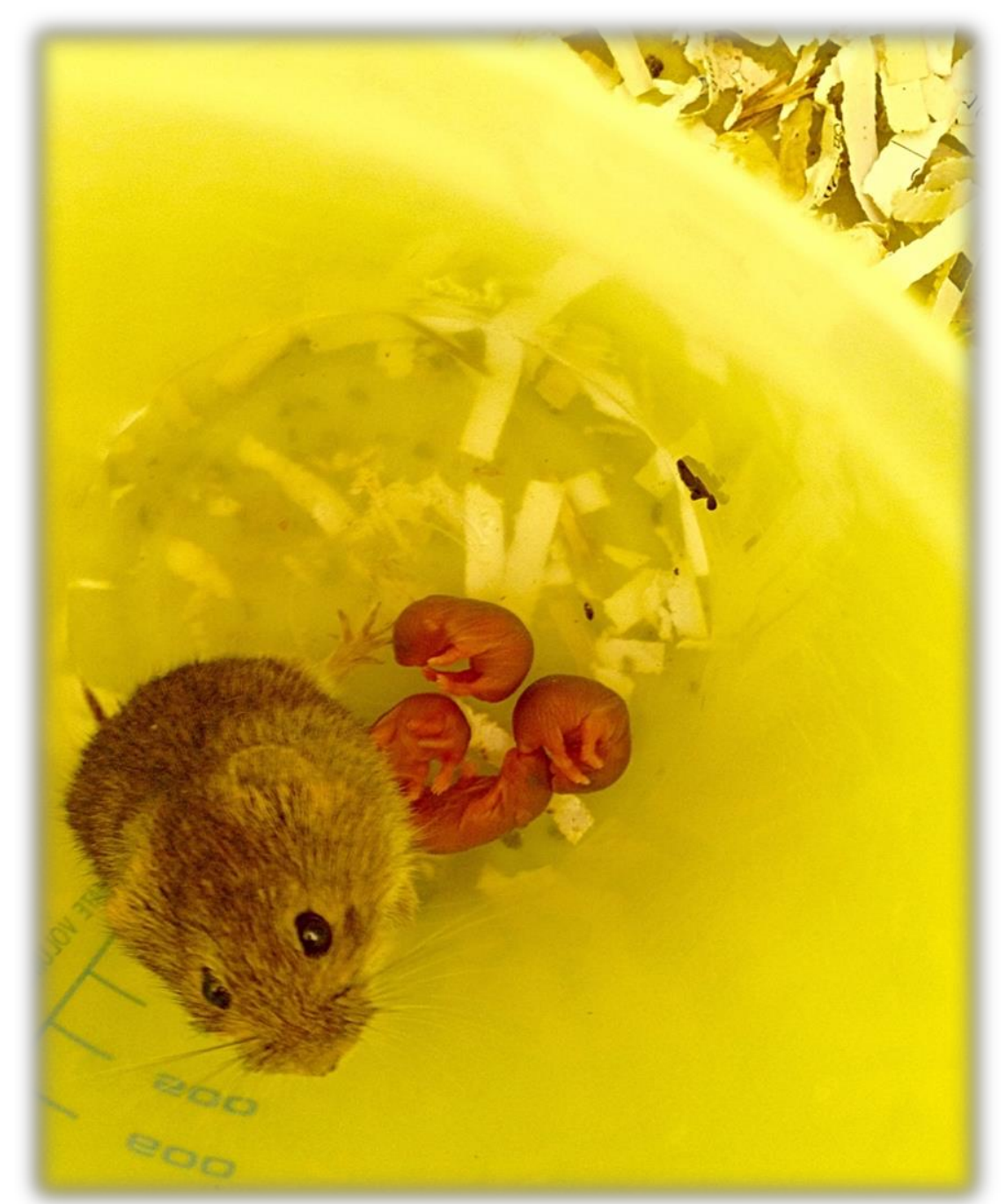




\section{Assessment of capture biases that influence population sex-ratio estimates in a fossorial rodent}

\section{Abstract}

Accurately estimating the adult sex-ratio (ASR) of animal populations is paramount for understanding demography and managing wildlife or assessing conservation. In species that are cryptic, elusive and difficult to sex visually, like many burrowing small mammals, this is, however, a challenging task. In such species, population density and composition are typically estimated using trapping devices, which can be highly susceptible to capture biases when these differ among sexes. Here, I compared ASRs obtained from two direct trapping methods in order to study if capture biases between sexes exist, and if so, find ways to correct them. The first estimate (ASR commonest study method for small mammals based on single trapping (ST) sessions and on catch-effort that does not take into account heterogeneity in capture. The second estimate $\left(A S R_{C M R}\right)$ was obtained from repeated capture sessions, individual marking and spatial capture-recapture (SCR) modelling. I applied both methods monthly for almost two years to study a population of free-ranging common voles (Microtus arvalis) inhabiting a $\sim 1.2$ ha alfalfa field. I found that both ASR estimates were only weakly correlated. Deviations between both estimates were significantly explained by season, differences in baseline probability of detection $\left(g_{0}\right)$ and movement $(\sigma)$ among sexes. ASR underestimated $\mathrm{ASR}_{\mathrm{CMR}}$ during summer, when males were more detectable in traps and when females moved less. The latter two parameters varied with vole 
reproduction and I show that considering season and the proportion of captured females that are breeding allows to better infer the ASR of the population using data from ST sessions. Given the potential biases associated with single trapping methods, I recommend using ASR derived from CMR and SCR modelling when studying small mammals such as voles, or to carefully study trapping biases in an attempt to correct ASR derived from ST.

Keywords: spatial capture-recapture modelling, sex-specific movement, detection probability, seasonality, reproduction, common vole, Microtus arvalis 


\section{Introduction}

The adult sex-ratio (ASR; proportion of adults of either sex) of a natural population is a key demographic parameter to population ecology (Godfray \& Werren, 1996; Kvarnemo \& Ahnesjo, 1996; Gyllenberg et al., 1997; Székely et al., 2014). Variations in ASRs have significant implications for our understanding of the dynamics, evolution or conservation status of animal populations (Armhein et al., 2012). ASRs can greatly vary among populations as well as among species (Navara, 2018), and play a major role in determining mating systems, sex roles or parental investment (see Kokko \& Jennions, 2008 for a review). Both theoretical and experimental studies have suggested that ASR variations impact sexual selection, behaviour, ecology and life history (Liker et al., 2013; Székely et al., 2014). In addition, ASRs have been shown to vary with population trends, making them useful early indicators of a species' population trajectory or conservation status (Ancona et al., 2017).

Variation in ASRs may arise because of skewed sex-ratios at birth (Székely et al., 2014), sex-biased dispersal or immigration (Clarke et al., 1997), differences in the maturation times of males and females (Stamps \& Krishnan, 1997; Donald, 2007) or sexual differences in juvenile or adult mortality (Clutton-Brock, 2007). These may be related to sex-specific costs of reproduction (e.g. Bennett \& Owens, 2002), a sex-biased selective removal from the population (e.g. trophy hunting) or predation rate (Roff, 2002; Berger \& Gompper, 1999), or sexual differences in parasitism rate and/or susceptibility to diseases (Moore \& Wilson, 2002). An understanding of the causes and implications of ASR variation can therefore provide important insights into the 
comprehension of ecological and evolutionary processes, as well as their implications for population demography and conservation (e.g. Donald, 2007; Grayson et al., 2014; Bessa-Gomes et al., 2004; Veran \& Beissinger, 2009; Wedekind et al., 2013). For instance, an overabundance of males can lead to low population growth and viability due to higher intrasexual competition and interference leading to the production of fewer offspring, with additional fitness costs on females due to aggressive behaviour or harassment (Dale, 2001; Clout et al., 2002; Clutton-Brock, 2007). By contrast, an excess of adult females could favour rapid population growth, and this may be especially relevant for social species in which females cooperate (e.g. Lambin, 1997), or for "boom-bust" species that quickly reach overabundance and become problematic (Krebs, 2013). Biased ASRs can thus create positive or negative feedbacks on population growth that can reinforce the decline or rise of animal numbers and increase the risk of population extinction when there is a deficit of females (Le Galliard et al., 2005; Rankin et al., 2011), or else favours demographic explosions.

In a variety of small rodent species ("boom-bust" rodents), strong multiannual population fluctuations are partly driven by changes in reproductive output, and their understanding requires a precise estimate of the number of breeding females in the population (Krebs, 2013). In most small mammals, males are the sex that disperses the most (Lambin et al., 2001), a process that can also contribute to bias the ASR in a population. High mortality rates in fluctuating small rodent populations can also shape the population ASR. There are some examples from field studies of differential pup mortality in nest sites in solitary vs groups of females in Microtus arvalis rates (33\% vs 63\%; Boyce and 
Boyce, 1988), or in high vs low kinship environments in Microtus townsendii (56\% vs $70 \%$; Lambin \& Yoccoz, 1998). Such differences are likely due to infanticide, but this is nearly impossible to document and measure in the field (Krebs, 2013). Infanticide by males is widespread in mammals and may be the main cause of pup mortality in some rodent populations (Palombit, 2012). For instance, because young in the nest are altricial, they are the primary subject to infanticide by adult females or males that view them as potential competitors for future living space (Krebs, 2013). In rodents, territorially in females seems to have evolved as a tactic to protect their young from intruding infanticide males (Wolff, 2007, 1993). All these tactics may result in a biased ASR related to differential mortality.

Obtaining reliable estimates of male and female abundance within a natural population, and therefore of its ASR, can be challenging with small mammals due to their complex behaviour (e.g. sex-specific interaction between trapping device and ecological characteristic of the animal). This is because abundance estimates are typically derived from methods that do not consider the heterogeneity in captures and are sensitive to sex-bias in capture rates. The two sexes may have different capturability due to differences in their behaviour and/or use of space (e.g. differences such as roaming time searching for mates, etc.). Heterogeneity of capture probabilities between males and females can strongly affect not only population estimates (Romairone et al., 2018), but also ASR estimates (McKnight \& Ligon, 2017). In voles, for instance, estimations of population ASR are usually carried out using naïve capture rate estimators that do not control for biases owing to the sex difference in capture probabilities or movements (Bryja et al., 2005; Donald, 2011). Such estimates should consider 
if one sex behaves differently towards traps, reacts differently to a previous capture event or moves over larger distances than the other (Bryja et al., 2005; Kikkawa, 1964; Yoccoz et al., 1993). Estimating ASR in animal populations can be challenging, but most difficulties can be alleviated through individual marking, capture-recapture estimates and demographic modelling (Kosztolányi et al., 2011; Armhein et al., 2012; Veran \& Beissinger, 2009).

Spatial capture-recapture (SCR) models have been recently developed to simultaneously consider factors affecting variations among individuals in capture probability, in order to obtain more accurate estimates of density and population structure (Efford, 2004; Royle et al., 2014, 2017). SCR can be easily adapted to small rodents like voles and is currently considered as the most quantitatively-robust method to be used for animal studies (Parmenter et al., 2003; Krebs et al., 2011; Gerber \& Parmenter 2015; Romairone et al., 2018). SCR modelling simultaneously estimates three structural parameters: (1) population density (D), (2) the basal probability of detection when an animal's home-range centre coincides with a trap location ( $\left.g_{0}\right)$, and (3) a scale parameter sigma $(\sigma)$ that describes how detection probability varies with the distance between an animal's activity centre and the trap location. Most importantly, these parameters can be estimated separately for different classes of individual (e.g. sexes). Considering and measuring potential differences in capture probabilities or movements between sexes, not only allows to obtain more precise abundance estimates for each sex, but also to study potential sources of bias that may affect naïve estimates of ASR derived from single trapping sessions (without marking and recapturing individuals). For instance, these single trapping sessions (i.e. catch-effort methods) are usually the commonest 
method used to estimate small mammals' abundance (e.g. Rodríguez-Pastor et al., 2016; Quéré et al., 2000; Krebs, 2013) because they are easier, faster (traps are usually deployed in the field for $24 \mathrm{~h}$ ) and less time-consuming than capture-mark-recapture methods. In addition, these single trappings also allow to collect other descriptive variables (e.g. sex, weight or age) or samples from the captured individuals. These reasons make single trapping sessions more suitable for large-scale monitoring of rodent pests.

In this study, I compared estimates of ASR derived from two direct methods in a free-ranging population of common vole (Microtus arvalis). The common vole is a small sized herbivore $(<100 \mathrm{~g})$ widely distributed from Northern Spain to the Middle East and Central Russia; it is one of the most abundant rodents in Europe and a facultative agricultural pest (Jacob \& Tkadlec, 2010). Some common vole populations are truly cyclical (Tkadlec \& Stenseth, 2001; Lambin et al., 2006), while other populations seem to fluctuate irregularly (Jacob \& Tkadlec, 2010). In northern and central Europe, multiannual population outbreaks are a common feature and occur regularly every 2-5 years (Cornulier et al., 2013). Some vole populations can cause important crop damage and economic loss during outbreaks in farmland areas (Jacob \& Tkadlec, 2010). In addition, they act as reservoir and amplifier of zoonotic diseases that have important public health implications (Han et al., 2015; Luque-Larena et al., 2015, 2017; Rodríguez-Pastor et al., 2017). Hence, there is a considerable, wide transdisciplinary interest in measuring and understanding demographic processes in outbreaking common vole populations. Precise measures of key demographic parameters such as ASR 
will help on the endeavour of understanding what triggers the rapid population growth phase that leads to vole outbreaks.

In this chapter, the main objective is to compare ASR estimates obtained from single trapping (ST) sessions with estimates from more intensive capturemark-recapture (CMR) sessions and spatial capture-recapture (SCR) modelling. For this purpose, I monitored the same alfalfa field during 21 consecutive months, with monthly assessments of ASR using both methods. I considered the ASR estimates obtained from CMR and SCR modelling as the most accurate (ASR $\mathrm{CMR}_{\mathrm{R}}$ ) and investigated how these estimates related those obtained through ST (ASRST). I show important deviations of ASR when using ST, and explored which factors may contribute to such observation, including: i) season, ii) sex-specific behaviours (i.e., sexual differences in basal detection probability and space use). I also investigated which factors (e.g. seasonality and the proportion of captured females) may contribute to sexual differences in detection probability or movement, in an attempt to find ways to correct ASR estimates obtained from ST sessions. 


\section{Material and methods}

\subsection{Study area and design}

The study was conducted in a 1.2 ha experimental field managed by the Universidad de Valladolid and located in Soto de Cerrato $\left(41^{\circ} 94^{\prime} \mathrm{N}, 4^{\circ} 42^{\prime} \mathrm{W}\right)$, Castilla-y-León region, NW Spain. The surrounding area is characterized by an intensive agricultural landscape dominated by cereal and alfalfa fields (Jareño et al., 2015; Rodríguez-Pastor et al., 2016). During spring 2015, the experimental field was planted with alfalfa (Medicago sativa, of the "Aragón" variety), which was neither irrigated nor treated with agrochemicals, and fenced (2.5 $\mathrm{m}$ tall wire mesh perimeter) to prevent public access while allowing small mammals to move through.

Estimating ASR requires capturing and sexing each captured vole phenotypically (visual inspection during handling). The two direct methods to estimate ASR were used sequentially (during the same week) and repeated at the same locations but alternating the ST positions every two months (see below). CMR live-trapping sessions lasted six consecutive days and were repeated every month approximately (capture sessions were separated by a three-week interval without trapping), starting in August 2016 until March 2018. The morning of the first day of each session was used for setting up the traps and was followed by five continuous daily capture occasions (trap-checks every 24 hours). In total, 21 CMR trappings sessions were conducted and used to model vole density and ASR (see below). The second method, ST, was conducted immediately after the CMR trapping session (i.e., on the sixth night after the first day of the session). 
a.

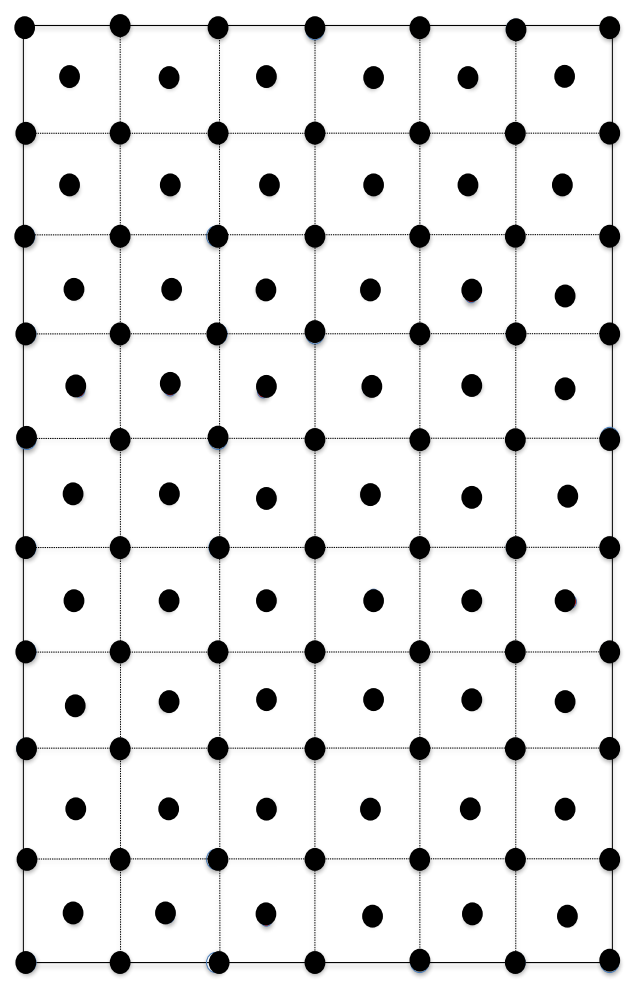

b.

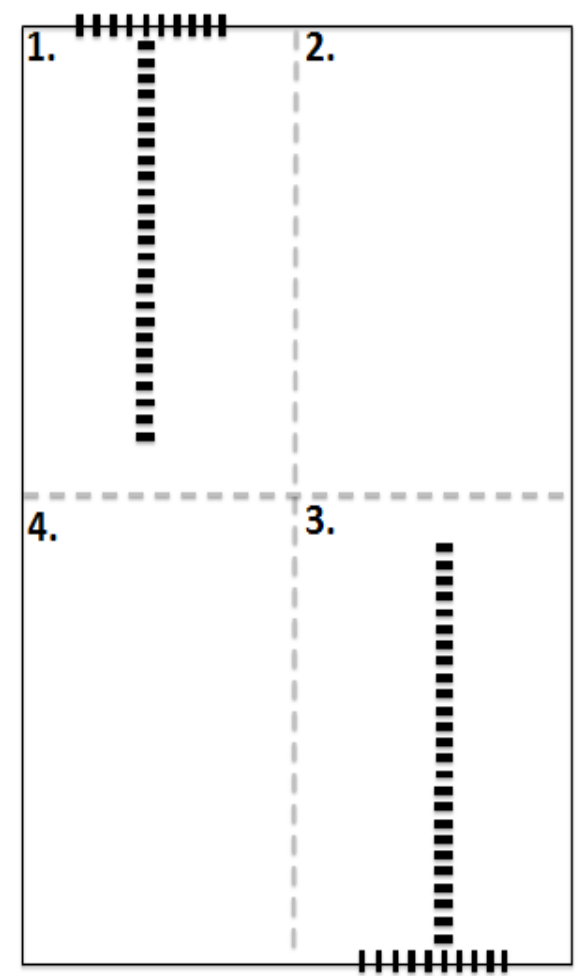

Figure 1. Sampling designs used for CMR and ST methods, (a) Trapping "grid" used for CMR sessions, which included 124 traps (black circles) on a grid size of $72 \times 135 \mathrm{~m}$. (b) Trapping design in "T" for ST sessions (same design as in previous studies by Rodríguez-Pastor et al., 2016, and Jareño et al., 2014). Two "T"-set of traps were operated in opposite corners, each including 35 traps that were set for $24 \mathrm{~h}$ (traps spaced every 2-m, with 10 traps on the margin and 25 traps inside the field). Every two months, the "T" sample units were alternated (using positions 1 and 3 position, and then 2 and 4 , and so on).

2.2. Density and ASR estimates obtained from capture-mark-recapture (ASRCMR)

\subsubsection{CMR trapping sessions}

Live trapping was conducted using a trapping grid of $72 \times 135 \mathrm{~m}\left(9,720 \mathrm{~m}^{2}\right)$ with 124 regularly spaced single-trap stations (Figure 1a). All traps consisted on a Sherman trap $(8 \times 9 \times 23 \mathrm{~cm}$; LFTA Sherman(C) coupled with a P.V.C nest box nest used to increase the survival of captured individuals during the coldest 
months (Romairone et al., 2018). P.V.C nest boxes were provided with bedding material (paper stripes) and baited with carrot and rodent lab chow (Global Diet 2018, Pellet $12 \mathrm{~mm}$, Teklad, Italy). Each trapping device (trap and nest) was covered by an inverted U-shaped concrete block to protect it from heavy rain, direct sunlight and to reduce the impact of frost (Romairone et al., 2018). Each vole captured for the first time was individually marked with a transponder (Glass tag, BIOGLASS 8625, 2x12mm, Ref: ICAR 941). I recorded several variables such as the trap location, vole sex and body weight. For females, I also recorded the following external reproduction signs: 1) pregnant or probably pregnant, based on body shape and mass (heavier than average for its size), 2) lactating with developed nipples, or 3) female giving birth in the trap. Voles were released at their capture site after processing. During subsequent recaptures, I simply recorded vole identity, weight and trap location. I calculated for each month a minimum proportion of reproductive females based on the abovementioned reproduction signs.

\subsection{SCR modelling}

For modelling, I used the package "secr" in R (see Chapter 2 for more detailed information) and defined an effective sampling area using a 30-m buffer for creating the state space $(S)$ and used a half-normal detection function for $\sigma$. I used a model selection process to specifically test if $\sigma$ (movement) varied according to sex, and if go varied according to sex, time (t) or vole behaviour (b) (see Chapter 1 and 2; Romairone et al., 2018 for more details). For each monthly CMR session, I thus fitted six candidate models that differed only in how these covariates affected $g_{0}$ or $\sigma$ (see Chapter 2). These models were 
compared and ranked using Akaike's Information Criterion (AIC) with a secondorder correction for small sample sizes (AICc; Burnham \& Anderson, 2002). I also considered the model with the lowest AICc value to be the best fit for the data and the most parsimonious. This selected model was used to obtain the best possible estimate of $A S R_{C M R}$. The output and selected models for each month are given in Table 1. For the sessions of December 2017, January 2018, February 2018 and March 2018, vole captures $(<10)$ and spatial recaptures were not enough for SCR modelling (see also Fauteux et al., 2018). Therefore, these four months (December 2017 to March 2018) were excluded from the analyses, considering hereafter 17 monthly CMR sessions. To explore sexual differences in $g_{0}$ or $\sigma$, I derived two ASR estimates from SCR models: one that corrected for differences between sexes in go or $\sigma$ (model with covariates) and one that did not; Null model (model without covariates influences (see Table 2). A Generalized Linear Model (GLM) was used to test for the association between both ASR $\mathrm{CMR}_{\mathrm{R}}$ estimates.

\subsection{Likelihood-ratio test for extra-compensatory heterogeneity}

When modelling in SCR, the simultaneous estimation of $g_{0}$ and $\sigma$ may be a potential source of heterogeneity, because both parameters are inter-related and may compensate each other (Efford \& Mowat, 2014). In some cases, unreliable estimates of $g_{0}$ and $\sigma$ may be returned by the best selected models, but without affecting the overall estimate of density. Efford \& Mowat (2014) recommended using a test to explore whether there is extra-compensatory heterogeneity with a given SCR model output. I conducted this test for each of the $17 \mathrm{CMR}$ sessions modelled, in order to validate the reliability of our 
estimates of $g_{0}$ and $\sigma . I$ used the two likelihood-ratio tests ("Test 1" and "Test 2") proposed by Efford \& Mowat (2014) to contrast the possible existence of extracompensatory heterogeneity. When evidence of extra-compensatory heterogeneity was detected, I did not consider the estimates of $g_{0}$ and $\sigma$ to be reliable, and therefore did not use them in our analyses.

\subsection{ASR estimates obtained from ST Sessions (ASR $\mathrm{ST}$ )}

ST sessions were carried out each month immediately after CMR trapping sessions. The experimental enclosure was divided in four equal areas of $36 \mathrm{x}$ $67.5 \mathrm{~m}\left(2430 \mathrm{~m}^{2}\right)$ coded clockwise from "1" (upper-left) to "4" (bottom-left) (Figure 1b). In each area, I set-up 35 Sherman traps (hereafter "sampling unit") spaced every $2 \mathrm{~m}$ and forming a "T"-shaped trapping line (Figure 2), with 10 traps placed along a $20 \mathrm{~m}$ transect line in the field margin, and 25 traps placed along a $50 \mathrm{~m}$ transect line perpendicular to the field margin and progressing towards the field centre. I followed the same methodology of the "sampling unit" applied in a long-term monitoring program still running in Castilla-y-Leon from 2009 (see Rodríguez-Pastor et al., 2016) due to its simplicity, less-effort than CMR and the broad applicability at large-scale monitoring for pest species. Two sampling units were simultaneously set-up for $24 \mathrm{~h}$ in diagonally opposed areas (i.e., trapping alternatively areas "1" and "3" or "2" and "4" every month). Each trap was coupled with a P.V.C nest box, as described above for CMR, provided with bedding and baited with carrot. Traps were set up in the morning, inspected after $24 \mathrm{~h}$ and subsequently removed. The captured individuals were released at their capture site. I used the number of voles trapped per 100 trap/24h as an abundance index (see Jareño et al., 2014; Mougeot et al., 2019). 
The ASRst was calculated as the proportion of females among all captured voles during a given session. As already mentioned, between December 2017 and March 2018, vole density was very low, and less than 5 voles were captured per session. The estimated ASR ST $_{\text {for }}$ these four months were considered as unreliable and were thus excluded.

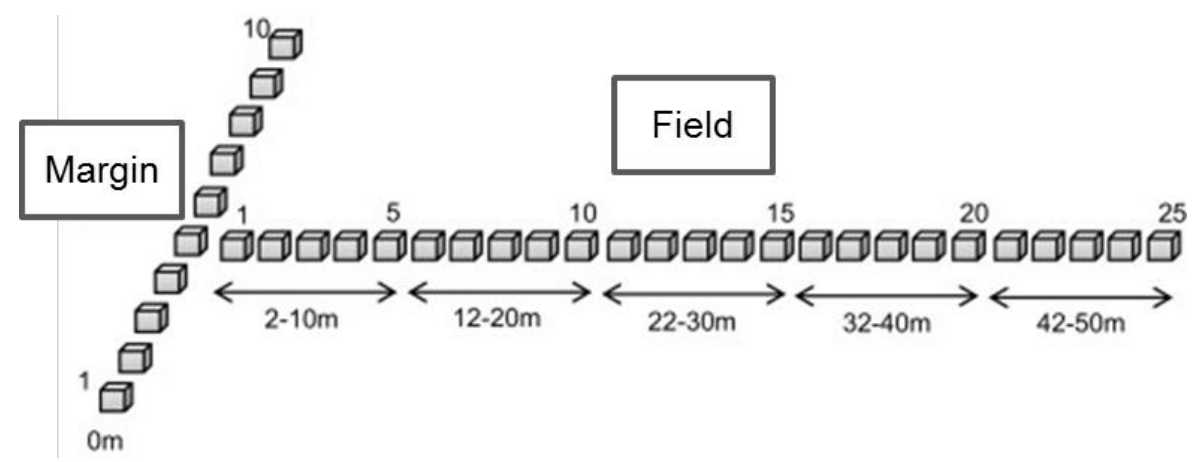

Figure 2. Disposition of traps in a given ST sampling unit ("T" lines with a total of 35 traps: 10 traps on the field margin and 25 traps within the field) (Figure adapted from Rodríguez - Pastor et al., 2016).

\subsection{Statistical analysis}

I used R v3.1.3 for all statistical analyses (R Core Team, 2015). I tested for associations between ASR $\mathrm{CMR}_{\mathrm{R}}$ and ASRst using GLMs. Both ASR estimates were square root-transformed prior to analysis. I explored deviations among the two ASR estimates by explaining $\mathrm{ASR}_{\mathrm{CMR}}$ with $A S R_{S T}$ and a number of factors or covariates, as candidate sources of bias. I specifically explored the influence of season (SEASON), D, go and $\sigma$ for males and females, and the proportion of reproductive females (REPRODUCTION). 


\section{Results}

\subsection{Extra-compensatory heterogeneity in SCR models}

I ran SCR models for 17 monthly CMR sessions (Table 1). I found evidence for extra-compensatory heterogeneity for two of these CMR sessions (January 2017 and April 2017; see Table 2, "Test 2" value <0.001). In other words, while density estimates were credible, the estimates of $g_{0}$ or $\sigma$ could be biased and were considered unreliable. In July 2017, "Test 2" was also significant but differences in ASR between the Null model and the best selected model with covariates indicate that extra-compensatory heterogeneity was not an issue for this particular month (Table 2). I thus excluded two sessions from our analyses of ASR variation that used $g_{0}$ or $\sigma$ as covariates. In any case, I also repeated the analyses using all CMR sessions in order to check that both data sets (15 vs 17 monthly ASR comparisons) of data gave similar results (see Appendix 3). 
उ के 음

을. 욜.

윽. 초 ᄚ

ชิ

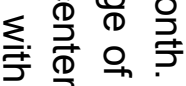

잉 宽

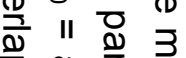

윰 ฏ

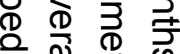

उัญ ญ

을 은 븐

은 후웡

守 무 这

ब

की

$\sum_{0}^{0}$

雨 $\stackrel{0}{\mathbb{1}} \stackrel{2}{\circ}$

으 웅. 옹

는 흑응 을

Ф

ब)

하 ₹

寺

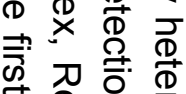

응

응 웅

읏 을 응

욱 움. 흘

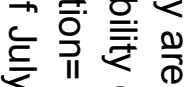

는 융

¿ 츨. 옥

川

ऽ क

음 ఫ्ष

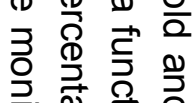

하웛 음 
Table 2. Tests for extra-compensatory heterogeneity estimated by fitting a two-class finite mixture. "Test 1" and "Test 2" are probability values for likelihood-ratio tests (variation in $\sigma$ given compensatory variation in $g_{0}$, and extra-compensatory variation in $g_{0}$, respectively). The table also reports the adult sex-ratio (ASR) derived from SCR models without covariate influence on $\mathrm{g}_{0}$ or $\sigma$ (Null model), or derived from the bestselected models (see Chapter 2 and Table 1) that allowed for an effect of behavior (b), time $(\mathrm{t})$ or sex on $\mathrm{g}_{0}$, or an effect of sex on $\sigma$.

\begin{tabular}{|c|c|c|c|c|c|}
\hline \multirow{2}{*}{ Year } & \multirow{2}{*}{ Month } & \multirow{2}{*}{ Test 1} & \multirow{2}{*}{ Test 2} & \multicolumn{2}{|c|}{$\mathbf{A S R}_{\mathrm{CMR}}$} \\
\hline & & & & $\begin{array}{c}\text { Model } \\
\text { with } \\
\text { covariates }\end{array}$ & $\begin{array}{c}\text { Null } \\
\text { model }\end{array}$ \\
\hline \multicolumn{6}{|l|}{2016} \\
\hline & $A \cup G$ & 0.005 & 0.05 & 0.454 & 0.419 \\
\hline & SEP & 0.019 & 0.90 & 0.351 & 0.405 \\
\hline & OCT & 0.001 & 0.02 & 0.540 & 0.510 \\
\hline & NOV & 0.000 & 0.01 & 0.721 & 0.690 \\
\hline & DEC & 0.000 & 0.01 & 0.538 & 0.522 \\
\hline \multicolumn{6}{|l|}{2017} \\
\hline & $J A N^{*}$ & 0.000 & $<0.001$ & 0.560 & 0.573 \\
\hline & FEB & 0.000 & 0.24 & 0.530 & 0.476 \\
\hline & MAR & 0.026 & 0.02 & 0.561 & 0.559 \\
\hline & $A P R^{*}$ & 0.000 & $<0.001$ & 0.561 & 0.545 \\
\hline & MAY & 0.001 & 0.01 & 0.722 & 0.647 \\
\hline & JUN & 0.001 & 0.12 & 0.529 & 0.413 \\
\hline & JUL & 0.000 & $<0.001$ & 0.624 & 0.523 \\
\hline & JUL2 & 0.470 & 0.94 & 0.521 & 0.520 \\
\hline & AUG & 0.000 & 0.04 & 0.540 & 0.470 \\
\hline & SEP & 0.210 & 0.67 & 0.637 & 0.600 \\
\hline & OCT & 0.020 & 0.02 & 0.594 & 0.560 \\
\hline & NOV & 0.370 & 0.17 & 0.687 & 0.690 \\
\hline
\end{tabular}

Both $A S R_{\text {CMR }}$ estimates (from the Null model and best selected model with covariates) were significantly positively correlated $\left(G L M: F_{1,13}=55.95\right.$, $p<0.001$ ), but with a Multiple- $r^{2}$ of 0.81 and Adjusted- $r^{2}$ of 0.79 , indicating that including covariates did improve the estimate of the ASR. 
3.2. Model outputs, temporal variations in ASR and other parameters

During 2016-2018, common vole abundance varied 10 -fold. The study period covered a decline phase from a maximum (estimate \pm SD) of $101 \pm 6.68$ voles/ha in August 2016 to a minimum abundance of $9.75 \pm 2.55$ voles/ha (November 2017; Figure 3a). Considering SCR model outputs, the ASR (i.e., ASR CMR $_{\text {) was }}$ consistently female-biased during most months (13 out of 15 months, excluding the two months with extra-compensatory heterogeneity), and male-biased for only two months (Figure 3b). By contrast, when considering ST methods, ASR ST was male-biased during most months (10 out of 17). The association between $A_{S R_{C M R}}$ and $A S R_{S T}$ was positive but only marginally significant $\left(F_{1,13}=3.38, p=\right.$ 0.08 , Multiple- $r^{2}=0.206$; Adjusted $-r^{2}=0.145$; Figure 4). Regarding potential differences in vole movement or probability of detection between sexes, I found that the movement parameter, $\sigma$, varied between maximum values of $12.45 \pm 1.27$ and $14.53 \pm 1.18$, and minimum values of $6.25 \pm 0.38$ and $4.60 \pm 0.41$, in males and females, respectively (Figure 3c). Sexual differences in the $\sigma$ parameter were selected for 13 out of 15 monthly CMR sessions (Table 1), indicating that male and female vole movement often differed. Males had higher $\sigma$ values than females for 13 out 15 of months (see Table 1).

The basal probability of detection parameter, $g_{0}$, varied over time (t) and between sexes, with maximum values of $0.45 \pm 0.13$ and $0.33 \pm 0.05$, and minimum values of $0.04 \pm 0.02$ and $0.05 \pm 0.01$, in males and females, respectively (Figure 3d). For nine months out of 15 , sex differences in go were detected; for five months out of $15, g_{0}$ values were higher in females than in males (i.e. in five months females were more detectable in traps than males). In one month, the go parameter also varied with time (t), i.e. varied during the five 
days of the capture session, for instance, due to a progressive adaptation of voles to the presence of traps over time. An influence of behaviour (b) on $g_{0}$ was also found for three months, i.e. the behavioural effect considered the degree of boldness of voles towards traps after their first capture (see Table 1 and Romairone et al., 2018 for detailed information). 
忢市

क्이 $\omega$

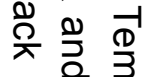

क्유 क음

ลิ

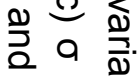

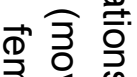

ฏ ญ

Ф

$\xi \stackrel{0}{c}$

的

(ब) 응

응 ज

ज) $\frac{1}{2}$

꿍ㅇ 옥

그소

क ल

(1)

क्ष

के 产 ()

* 응 응

क

ญำ ㅇํำ

穴 西 $\frac{\delta}{\sqrt{D}}$

उ

응

क ई

ミ. 穴

을

(1) $\stackrel{\text { ले }}{\stackrel{0}{口}}$

这

กุ๊ำ

은

(1) 응

흘

ญे

응 흐

궁 뭉 흐

ग 융

की ठग

完䒝

그음

ㅁํㅇำ 음

융

응 울

몰 을

$\underset{\infty}{\stackrel{n}{\infty}}$

₹ㅇㅇㅛ 용
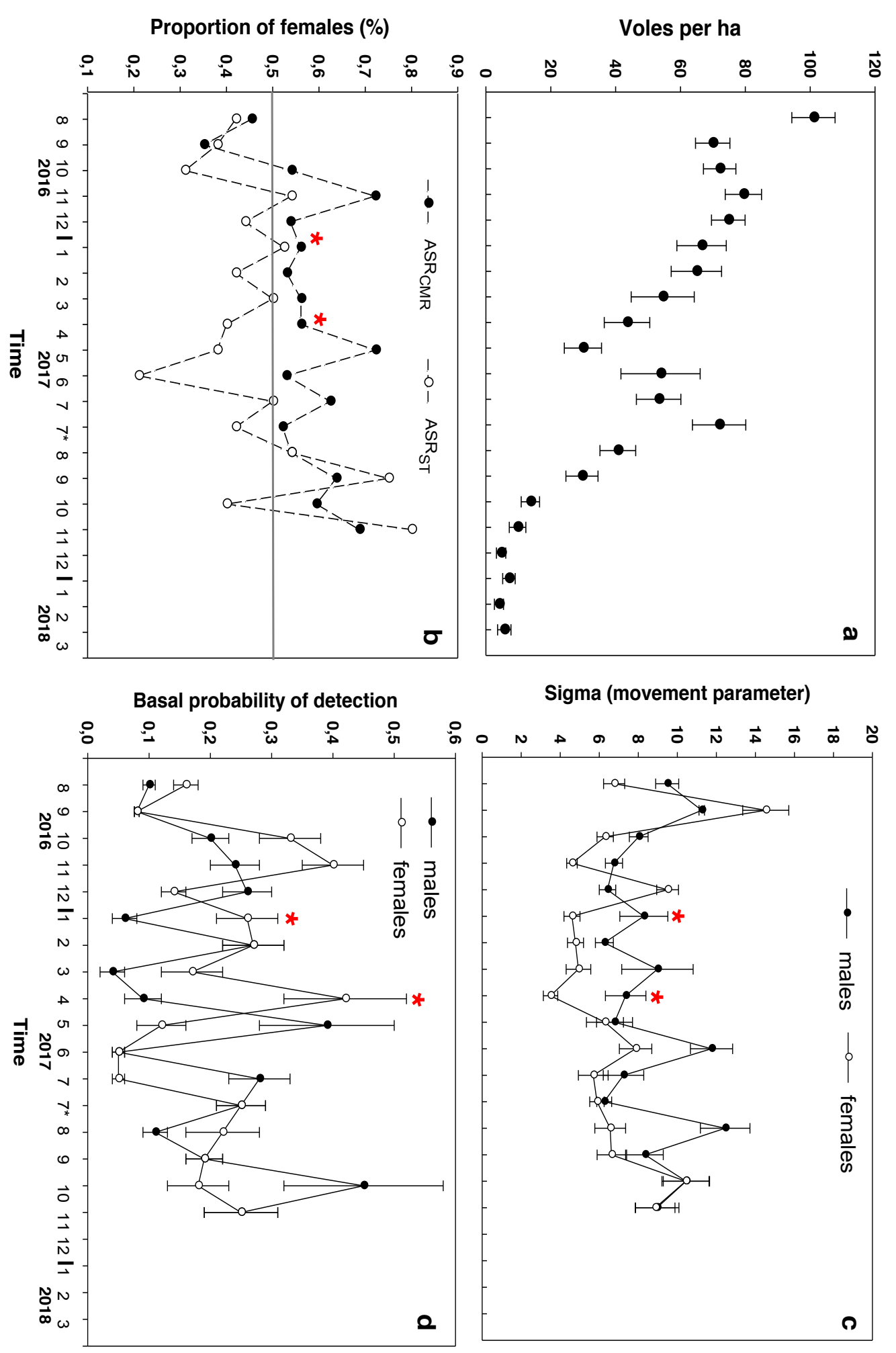


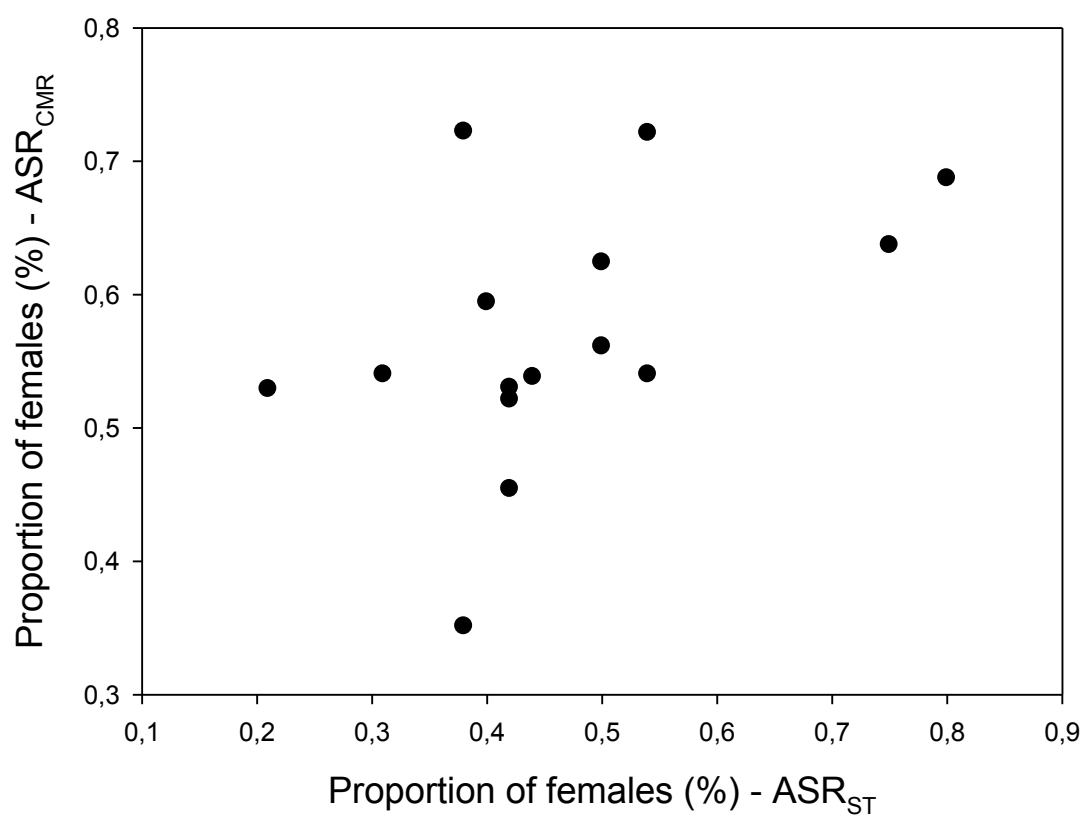

Figure 4. Relationship between the proportion of females obtained by SCR modelling (CMR) and by ST method.

3.3. Factors explaining deviations between $A S R_{C M R}$ and $A S R_{S T S}$

\subsubsection{Season}

I found that the residuals of the relationship between $A S R_{C M R}$ and $A S R_{S T}$ significantly varied between seasons (model: $A R_{C M R} \sim A S R_{S T}+$ SEASON; $F_{4,9}=6.65 p=0.008$, Multiple- $r^{2}=0.747$, Adjusted $\left.-r^{2}=0.635\right) . \quad A S R_{C M R}$ prediction was underestimated in summer $(p=0.01)$ and to a lower extent in winter $(p=0.09 ;$ Figure 5). 


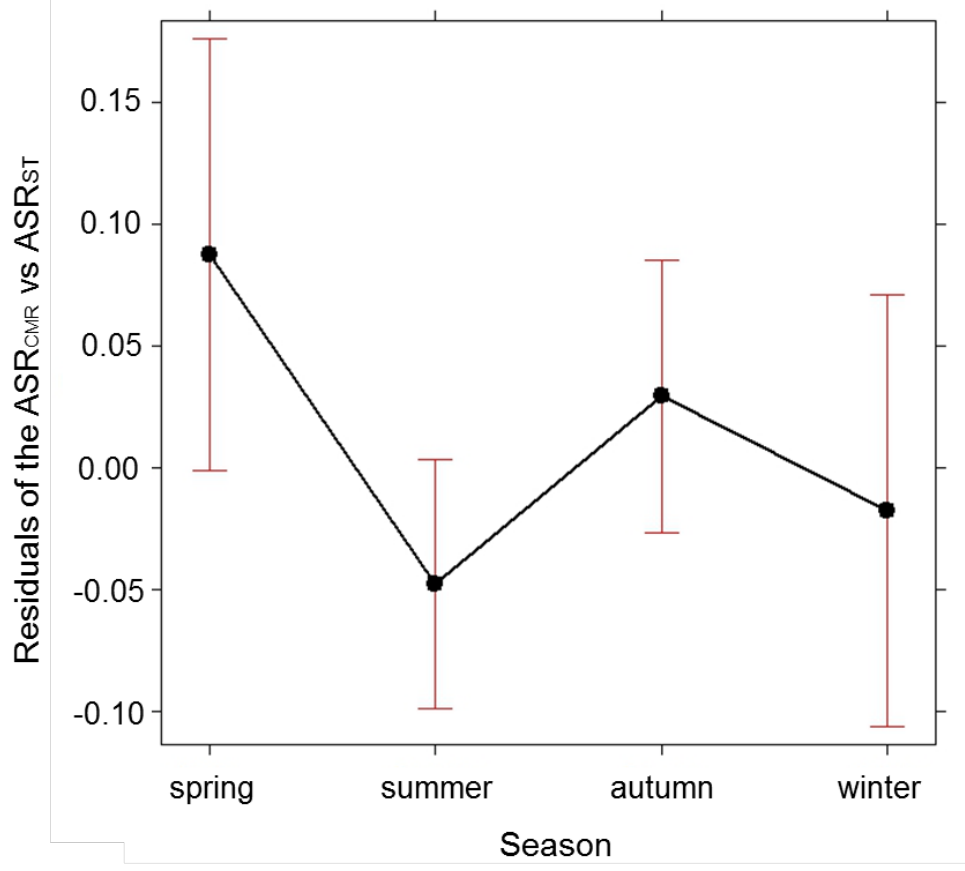

Figure 5. Linear model output showing the seasonal variation in the residuals from the association between $\mathrm{ASR}_{\mathrm{CMR}}$ and $\mathrm{ASR}_{\mathrm{ST}}$.

3.3.2. Vole density and vole behaviour ( $\sigma$ and $\left.g_{0}\right)$

Using univariate analyses to explain differences in prediction between ASR $R_{C M R}$ and $A S R_{S T}$, I found a significant effect of male $g_{0}\left(F_{2,12}=5.22, p=0.02\right.$, Multiple$r^{2}=0.465$, Adjusted- $\left.r^{2}=0.376\right)$, female $\sigma\left(F_{2,12}=4.25, p=0.04\right.$, Multiple- $r^{2}=0.414$, Adjusted- $\left.r^{2}=0.317\right)$ and vole density $\left(F_{2,12}=3.04, p=0.08\right.$, Multiple- $r^{2}=0.336$, Adjusted- $r^{2}=0.226$ ). The other variables (female $g_{0}$ and male $\sigma$ ) were not significant (Table 3). ASR $\mathrm{ST}_{\mathrm{S}}$ tended to be underestimated $\mathrm{ASR}_{\mathrm{CMR}}$ at higher vole density (Figure 6a) and was significantly underestimated when females moved less (lower $\sigma$; Figure $6 b$ ) or when males had a lower probability of detection (Figure 6c). 
Table 3. Results of the univariate analyses to explain the residuals from the association between $\mathrm{ASR}_{\mathrm{CMR}}$ and $\mathrm{ASR}_{\mathrm{STS}}$ (square root-transformed). Significant variables are highlighted in bold.

\begin{tabular}{lcccc}
\hline \multicolumn{1}{c}{ Models } & $\mathbf{F}$ & $\boldsymbol{p}$ & Multiple-r $^{\mathbf{2}}$ & Adjusted-r $^{\mathbf{2}}$ \\
\hline $\mathrm{ASR}_{\mathrm{CMR}} \sim \mathrm{ASR}_{\mathrm{ST}}+$ Density & $\mathrm{F}_{2,12}=3.04$ & 0.08 & $\mathbf{0 . 3 3 6}$ & $\mathbf{0 . 2 2 6}$ \\
$\mathrm{ASR}_{\mathrm{CMR}} \sim \mathrm{ASR}_{\mathrm{ST}}+\sigma$ females & $\mathrm{F}_{2,12}=4.25$ & 0.04 & $\mathbf{0 . 4 1 4}$ & $\mathbf{0 . 3 1 7}$ \\
$\mathrm{ASR}_{\mathrm{CMR}} \sim \mathrm{ASR}_{\mathrm{ST}}+\sigma$ males & $\mathrm{F}_{2,12}=3.00$ & 0.08 & 0.333 & 0.222 \\
$\mathrm{ASR}_{\mathrm{CMR}} \sim \mathrm{ASR}_{\mathrm{ST}}+\mathrm{g}_{0 \text { females }}$ & $\mathrm{F}_{2,12}=1.96$ & 0.18 & 0.247 & 0.121 \\
$\mathrm{ASR}_{\mathrm{CMR}} \sim \mathrm{ASR}_{\mathrm{ST}}+\mathrm{g}_{\text {o males }}$ & $\mathrm{F}_{2,12}=5.22$ & 0.02 & $\mathbf{0 . 4 6 5}$ & $\mathbf{0 . 3 7 6}$ \\
\hline
\end{tabular}




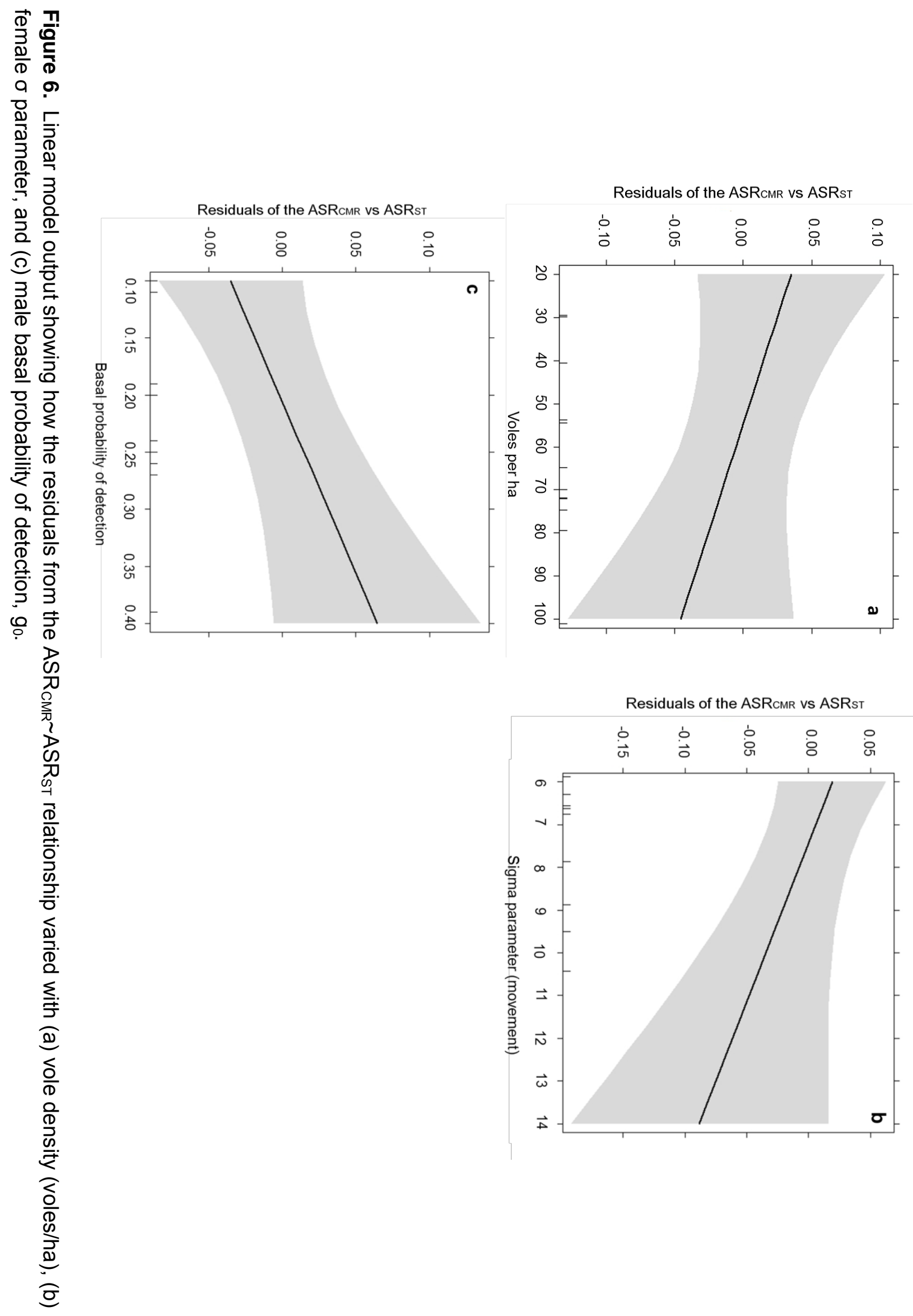


When combining these covariates (multivariate analysis), the best model explaining the residuals of the relationship between $A S R_{C M R}$ and $A S R_{S T S}$ included the variables season, female $\sigma$ and male $g_{0}\left(F_{6,8}=22, p=<0.001\right)$, and explained more than $90 \%$ of the variation (Multiple- $r^{2}=0.942$, Adjusted$\left.r^{2}=0.900\right)$.

3.3.3. Variations in $\sigma$ and go according to season, reproduction and density

The parameter $\sigma$ in females did not vary with season $\left(F_{3,11}=0.66, p=0.58\right.$, $\left.r^{2}=0.154\right)$ or with vole density $\left(F_{1,13}=0.33, p=0.57, r^{2}=0.02\right)$, but tended to decrease with an increasing proportion of reproductive females $\left(F_{1,13}=2.38\right.$, $p=0.14$, Multiple- $r^{2}=0.154$, Adjusted $-r^{2}=0.08$ ). Similarly, male $\sigma$ did not vary between seasons $\left(F_{3,11}=0.39, p=0.76, r^{2}=0.09\right)$ or with density $\left(F_{1,13}=0.77\right.$, $\left.p=0.39, r^{2}=0.05\right)$, and also tended to decreased with the proportion of females reproductive $\quad\left(F_{1,13}=2.18, \quad p=0.16, \quad\right.$ Multiple- $r^{2}=0.143, \quad$ Adjusted- $\left.r^{2}=0.07\right)$. Therefore, both sexes tended to move less when breeding increases. 


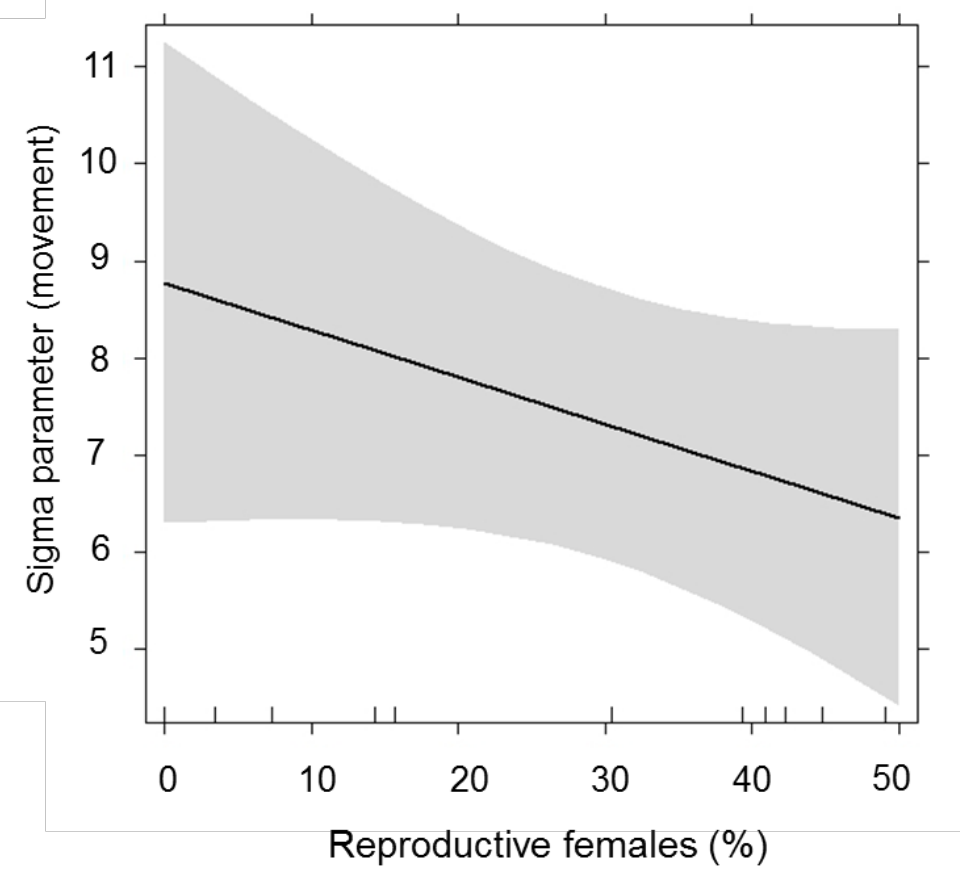

Figure 7. Relationship between female $\sigma$ parameter and breeding activity among females (\% of reproduction).

The parameter $g_{0}$ in females did not vary with density $\left(F_{1,13}=0.23, p=0.63\right.$, $\left.r^{2}=0.01\right)$ or proportion of reproductive females $\left(F_{1,13}=0.34, p=0.56, r^{2}=0.02\right)$, but tended to vary with season $\left(F_{3,11}=2.37, p=0.12\right.$, Multiple- $r^{2}=0.393$, Adjusted$r^{2}=0.227$; Figure 8). Male $g_{0}$ did not vary with season $\left(F_{3,11}=0.95, p=0.44\right.$, $\left.r^{2}=0.20\right)$ or proportion of reproductive females $\left(F_{1,13}=0.004, p=0.94, r^{2}<0.01\right)$, but tended to decrease with vole density $\left(F_{1,13}=2.90, p=0.11\right.$, Multiple- $r^{2}=0.18$, Adjusted- $\left.r^{2}=0.109\right)$. 


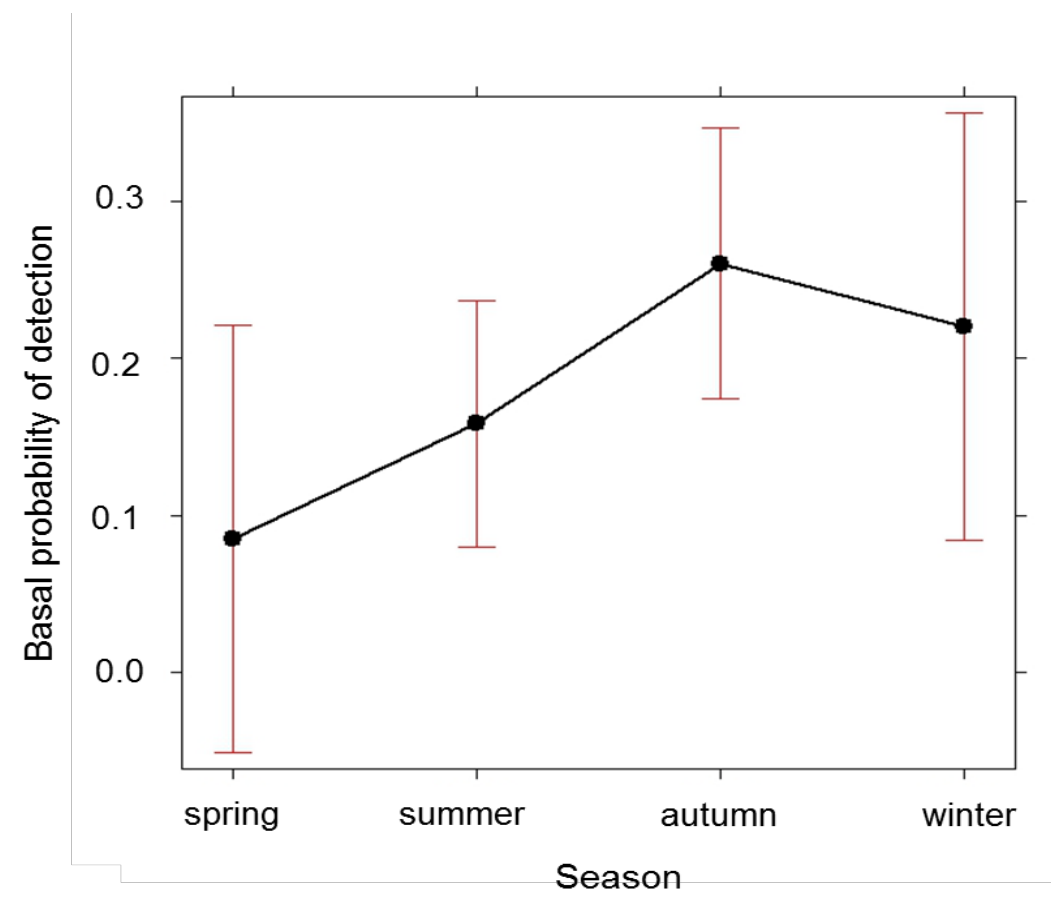

Figure 8. Variation of female basal probability of detection, $g_{0}$, according to season.

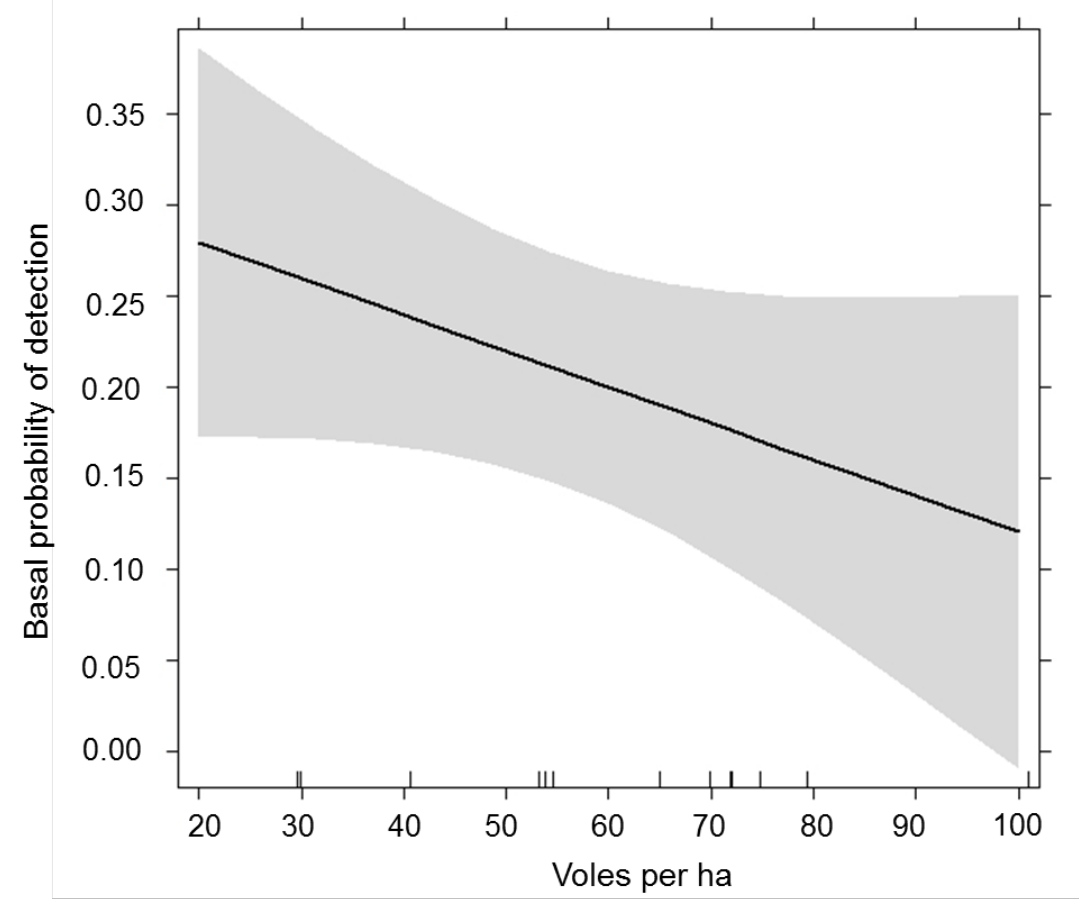

Figure 9. Relationship between male basal probability of detection, $g_{0}$, and population density. 
Given these findings, I tried to predict ASR CMR from ASR $_{\text {ST }}$ by using not only season, but also information on the proportion of reproductive females, which can be assessed during single trapping sessions. The resulting model (model: $\mathrm{ASR}_{\mathrm{CMR}} \sim \mathrm{ASR}_{\mathrm{STS}}+\mathrm{SEASON}+\mathrm{REPRODUCTION}$ ) explained $75 \%$ of variation $\left(F_{5,9}=5.52, \quad p=0.01\right.$, Multiple- $r^{2}=0.754, \quad$ Adjusted $\left.-r^{2}=0.617\right)$, and allowed to improve prediction of ASR in the population knowing only the season and the proportion of females reproducing.

Table 4. Table of coefficients of a linear best model with season and reproduction as best predictors.

\begin{tabular}{lcccc}
\hline \multicolumn{1}{c}{ Factor } & Estimate & $\mathbf{S E}$ & $\mathbf{t}$ & $\mathbf{P}$ \\
\hline Intercept & 0.35879 & 0.06805 & 5.272 & 0.000512 \\
ASRST & 0.44797 & 0.12449 & 3.598 & 0.005763 \\
Season_spring & 0.06323 & 0.06187 & 1.022 & 0.333471 \\
Season_summer & -0.11674 & 0.03887 & -3.003 & 0.014872 \\
Season_winter & -0.06090 & 0.05204 & -1.170 & 0.271953 \\
Reproduction & 0.15934 & 0.08455 & 1.885 & 0.092117 \\
\hline
\end{tabular}

${ }^{*} A S R_{S T}=$ proportion of females captured in the population.

${ }^{* *}$ Reproduction $=$ proportion of reproductive females. 


\section{Discussion}

An accurate and reliable estimation of ASR in an animal population provides important information relevant for species management and conservation (Donald, 2007; Lee et al., 2011). Methods based on marking individuals (CMR methodologies) are the most accurate to estimate the ASR (reviewed by Ancona et al., 2017), but require more effort than single trapping (ST) methods, in which fewer traps are usually deployed over a shorter period (Fauteux et al., 2018). Thus, understanding and correcting potential bias in ASRs derived from the simpler ST methods would be an important step forward.

In the present study, I showed that during most months the $A_{S R} R_{C M R}$ was consistently female-biased when estimated by CMR (Figure 3b). A femalebiased ASR was previously observed (see Chapter 1 or Romairone et al., 2018) during the months of May and June of 2016, and can now be confirmed over time, or at least during the whole study period covering a pronounced vole population decline (Figure 3a). By contrast, the ST method revealed a male-

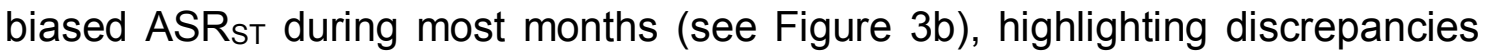
between the two methods used to estimate ASR. In fact, ASRst was malebiased in at least 10 (59\%) months out of 17 months of study. By contrast, in ASR $_{\text {CMR }}$ modelled data, I only observed two months $(13 \% ; n=15)$ with a malebiased ASR. These discrepancies between ASR $_{\text {CMR }}$ and ASR ST $_{\text {suggest the }}$ existence of biases imposed by the trapping method and common vole behaviour in the ASR estimates (see below). Indeed, the $\sigma$ parameter (movement) and the basal probability of detection, $\left(g_{0}\right)$ often differed among males and females (as also shown in Chapter 1; Romairone et al., 2018). 


\subsection{Potential bias imposed by the different trapping method}

In the present study, bias in ASR potentially come from discrepancies between both trapping methods and the amount of time the traps were deployed in the field (trapping grid $=5$ days vs. T-line sampling $=1$ day). In a study with common voles, Gauffre et al., (2009) carried out a similar methodologies of sampling common voles. They sampled individuals directly from the colonies placing baited traps close to the burrows and then, they used line-trapping sampling and surveyed for $24 \mathrm{~h}$. Similar to the thesis results, Gauffre et al., (2009) showed that with their line-trapping method, captures were male-biased, whereas with the colony trapping, captures were female-biased. Such differences can be explained by sexual differences in the behaviour in common voles (e.g. movements) and highlight that sex-ratio is truly difficult to measure accurately (Bryja et al., 2005). Furthermore, it is remarkable that ASR CMR $_{\text {( }}(5$ days of trappings and modelled data) were consistently female-biased, while $\mathrm{ASR}_{\mathrm{ST}}$ (1 day of trapping) were more male-biased. Discrepancies between both methods could be due to differences in the spatial placements of the traps (trapping grid with 124 traps covering the whole field vs T-trapping lines with 35 traps covering only a portion of the field. Because vole distribution and abundance are not uniform (see Romairone et al., 2018), the inclusion/exclusion of vole colonies may have important implications on the proportion of sexes captured when only a portion of the field is sampled. Therefore, the numbers of males and females trapped directly in a population should not be used for assessing population proportions as these without taking into account sex differences (heterogeneity) in capture probability and spatial activity (Kikkawa, 1964; Yoccoz et al., 1993; Bryja et al., 2005). 
The discrepancies in the ASR estimated with both methods were partially related to season in the study. This seasonal pattern of population sex-ratio is not totally in agreement with the results of Bryja et al., (2005) where the proportion of males was highest both in winter and spring and then dropped gradually as the breeding period progressed. In this case, the $A S R_{C M R}$ was underestimated during summer and to a lower extent during winter. This seasonal pattern of vole population sex-ratio may be an important population property driven by sexual differences in vole behavior. The parameters derived from the SCR modeling can inform about vole behavior and help understand biases in ASR estimates. The $\sigma$ parameter that allows to infer movement ( home range; $\mathrm{HR}$ ) and possible differences between males and females. The parameter go describes the probability of detection by sex (Royle et al., 2014, 2017). These are two important ecological parameters to consider, for instance, if males have a higher capture probability than females, or move over larger distances, their proportion in the sample would inevitably overrepresented (Bryja et al., 2005).

\subsection{Behavioral differences between sexes}

In the present study, at least the $50 \%$ of the individuals were captured once in the experimental plot (see Appendix 3 - S2 Supporting Information). Although I did not apply statistics for modelling specific demographic parameter such as dispersal or survival rates, I can infer that those individuals captured only once were transients (i.e. opposed to residents) which emigrate (i.e. disperse) to other places or merely were dead. In a study aimed to investigate the genetic structure and the sex-specific dispersal patterns of M. arvalis, Gauffre et al., (2009) found that females were strongly philopatric (forming kin-clusters within 
colonies) and that dispersal was overall male-biased at a local scale. They also showed that female dispersal between colonies was not a common process due to the impossibility to settle down in a place taken for other groups of related females. Colonization events consisted of larger movements of females to a new available area with no competition with relatives. In relation to male dispersal, Gauffre et al., (2009) found that males migrated continuously from colony to colony to reproduce. I have found here, that ASRSt tended to be underestimated when females moved less and when males had a higher probability of detection.

According to the results of Gauffre et al., (2009) I can find a biological explanation to an influence vole behavior variable ( $g_{0}$ of males and $\sigma$ of females) on sex ratio bias. If female voles are kin-clustered within a colony (Gauffre et al., 2009), these should not move much far away from the colony perimeter. This agrees with the present study finding that female movement was reduced when the proportion of breeding females was higher. Regarding an influence of the go of males, males may be moving continuously from colony to colony to reproduce and therefore, be more likely to be detected by trapping (increased $g_{0}$ ) during reproduction times. It is important to take into account that sex-specific heterogeneities of capture probabilities were shown to be among the factors having the biggest potential to bias population ASR (Bryja et al., 2005, Drickamer et al., 1999, McNight \& Ligon, 2017). For instance, in the present study the capture rates of females were almost three times higher than those of males during the most female-biased ASR months (up to 0.72 or $\sim 3: 1$, i.e. three females per male). 
I have shown here, that the combination of several variables such as season, the parameter sigma $(\sigma)$ of females and the probability of capture $\left(g_{0}\right)$ of males explained the discrepancies between $A S R_{C M R}$ and $A S R_{S T}$, and therefore are important sources of bias in ASR estimates derived from single capture events. Understanding variation in $\sigma$ and $g_{0}$ could therefore allow to correct ASR estimates derived from the simplest method based on single trappings. For this purpose, I studied how these parameters varied with vole density and reproduction. I found that both female and male of common vole tended to decrease their movements $(\sigma)$ when the proportion of breeding females in the population increased (up to $59 \%$ - the maximum of breeding females for a given month in all study period). When using the whole dataset (i.e. not excluding the two months with evidence of extra-compensatory heterogeneity), a negative association between the $\sigma$ of females and the $\%$ of reproduction was significant (see Appendix 3 - S1 Supporting Informsation). Females thus appear to decrease their movement when reproducing, as suggested before for common voles (Romairone et al., 2018) and meadow voles Microtus pennsylvanicus during parturition and early lactation (Madison, 1978). In other microtines, Lambin (1997) showed that breeding female of Townsend's voles Microtus townsendii were almost sedentary once they started to reproduce. These observations support the assumption that females moved less that males when reproducing and are likely less detectable by trapping, leading to underestimated ASR with single trapping (ST) methods.

Regarding male movement ( $\sigma$ parameter), it is known that in small mammals such as voles, males usually show a polygynous behaviour (Krebs, 2013), and have large home ranges (HRs) that overlap partly or entirely with 
adult females (Waterman, 2007; Krebs, 2013). Monogamy is usually rare (e.g. Microtus ochrogaster: Getz et al., 1993). I found that male common voles also tended to decrease their movement with an increasing proportion of female reproducing (reproductive behaviour). It is known that HRs size could also vary among seasons: it can be reduced when females give birth (e.g. Amori et al., 2000; Verhagen et al., 2000), and pregnant or lactating females are more sedentary than non-reproductive ones (Koskela et al., 1997). In our study the minimum values of $\sigma$ for males and females were $6.25 \pm 0.38$ (corresponding to an estimated HR of $734 \mathrm{~m}^{2}$ ) in summer (July 2017) and $4.60 \pm 0.27$ (HR: $398 \mathrm{~m}^{2}$ ) in autumn (November 2016), coinciding reproductive periods (with $55.31 \%$ and $42.25 \%$ of breeding females, respectively). The maximum $\sigma$ parameter and HRs values for males were 12.45 $\pm 1.27\left(\mathrm{HR}: 2915 \mathrm{~m}^{2}\right)$ and for females $14.53 \pm 1.18$ (HR: $3970 \mathrm{~m}^{2}$ ). These findings are similar to those described in other Microtus spp. such as M. oeconomus (male HR averaged $804 \mathrm{~m}^{2}$, with maximum 2756

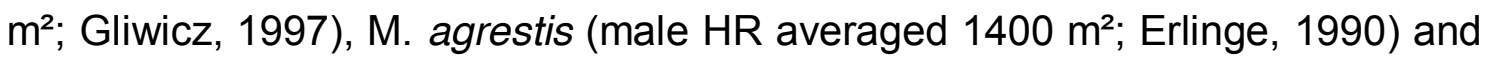
Arvicola sapidus (HRs up to $2858 \mathrm{~m}^{2}$; Pita, 2010). In M. agrestis (Loughran, 2006) males HRs either did not vary during the breeding season, or increased during breeding time (Jensen et al., 2012) depending on population density. In low density populations, individuals may move larger distances and occupy larger territories without competition (Sandell et al., 1991). 


\section{Conclusions}

In conclusion, I have found a consistently female-biased ASR in the study vole population, which has implications for understanding vole demography, as a female surplus can contribute to rapid population growth leading to demographic explosions (Krebs, 2013). This female surplus did not halt the decline phase that occurred during the study, suggesting that other processes drive the population decline (mortality, immigration). I have also shown that ASR estimates derived from single trapping sessions should be taken with caution, because they are likely affected by a number of biases. These were in part due to seasonal effects and variations in vole movement and basal probability of detection in traps. These factors should be further studied in order to correct ASR estimates derived from single capture sessions. In the context of the present study (a declining vole population, from medium -100 voles per hato low densities $<5$ voles per ha) I have shown that some of the biases can be dealt with by considering the correcting factors season and vole reproduction (\% of reproductive females), as both variables captured some of the variations in the movement and detectability of male and female voles that affected ASR estimates. Incorporating these is thus recommended to improve estimates of ASR derived from single trapping of common voles. It would be desirable repeat a study like this during an increasing vole density phase, in order to assess if the factors affecting ASR biases could be different then. This study was based on the results of one experimental field in which it would also be desirable to apply the same methodology in other habitats with the aim to check whether the results are consistent in space and time. Finally, its study is one of few (see Casula et al., 2018) that made use of parameters other than density or 
population composition that can be derived for SCR modelling (here sexspecific $\sigma$ and go parameter values that informed about differences in the movements and detection probabilities of males and females). I encourage more use of such information to improve our understanding of the ecology and population dynamics of animals studied with CMR and SCR modelling. 


\section{Discussion}

\subsection{Brief summary of the main message of this thesis}

A main aim of this thesis was to improve current methods used in the field to estimate common vole density and key population parameters such as sexratio. To do so, requires a better understanding of the heterogeneities in capturability or detectability of voles, either using capture devices or activity signs, and of any potential bias associated with each survey method. The first requirement to achieve this aim is to adapt the best available methodology (Capture-Mark-Recapture -CMR- and Spatial Capture-Recapture -SCRmodelling) to accommodate specific characteristics of small rodents like common voles. Once this robust baseline method had been developed, the subsequent step consisted in validating and calibrating simpler methods based on vole activity signs (VAS) or single trapping sessions (STSs), which allow for rapid and simpler monitoring. As a result, it was possible to validate and calibrate an indirect surveying method based on activity signs that is routinely used for the large-scale monitoring of common vole populations. Additionally, an assessment of the potential bias in the capturability of females and males in a population when using STS was done. From revealing these biases, I proposed potential ways of correcting population sex-ratio estimates obtained using this common vole monitoring method.

Throughout the following paragraphs, I will discuss several aspects which have been shown to be related to the detection probability of individuals and highlight the main factors that can affect detectability. Understanding which factors can influence detection will allow researchers to be taken these effects 
into account when design future monitoring methods. Doing so, these potential biases will be reduced, enabling more robust and reliable ecological studies. For example, with threatened species like Cabrera's vole (Microtus cabrerae), having accurate estimates of population size should lead to better informed decisions being made (e.g. Sabino-Marques et al., 2018).

With this in mind, I discuss both technical and statistical improvements that I have applied in order to improve small mammal's knowledge, such as voles (Chapter 1) population size estimates and other key ecological parameters. This was achieved through the application of SCR, which subsequently enabled adult sex-ratio to be estimated (Chapter 3). Moreover, the application of SCR has also allowed the calibration of the alternative methods which are widely used to monitor wildlife populations without having to capture or handle individuals (non-invasive sampling methods; Chapter 2). Finally, I discuss the potential disadvantages that may arise between the SCR models and the ecology of our study species and how these should be treated in the future (e.g. extensions for SCR modelling), as well as suggest potential lines of research that can be further developed.

In summary, technical improvements that improve the detection of individuals in the field (e.g. adjusting the placement of different capture devices to the movement of the species of study) and the application of the state-of-art of modeling (e.g. SCR statistical models which take into account the heterogeneity in capture rate) will allow better and more precise estimates. When such information can be estimated, they open a range of possible ecological questions for future and current studies. Such questions include investigating adult sex-ratio within a population (chapter 3 ), where a precise 
estimate of the number of females can inform on the viability of a population or allow better understanding of demographic processes. Further, from calibrating non-invasive monitoring methods with SCR allows for informed monitoring leading to rapid, sufficiently detailed and timely information that may help resolve human-wildlife conflicts (chapter 2). Consequently, the combination of the novel results, presented within this thesis, offers the opportunity to update, increase and improve current monitoring methods and enabling opportunities to achieve better basic ecological knowledge of a pest species, the common vole.

\subsection{The importance of detection probability for population monitoring}

Detection rate is the likelihood of correctly identifying a species as being present within a site, dependent on that species actually being present in situ. This ability to correctly detect individuals is of vital importance in any effort to monitor wildlife populations (O'Connor et al., 2017). For instance, over the last few decades, biologists and researchers have developed a multitude of a new techniques and applied the most advanced technology in order to increase detectability for rare, elusive or cryptic species, from those living in water using eDNA as powerful method for assessing the presence and the distribution of aquatic species (Mauvisseau et al., 2017), using drones for Florida manatees (Martin et al., 2002) and camera traps for terrestrial mammals such as rare carnivores (Franklin et al., 2019). For small mammals, however, the most accurate form of detection remains standardized trapping (Harkins et al., 2019). The problem arises when detection is imperfect, which is usually the rule, whereas other indications must be followed to take this into account, in order to 
avoid biases and generating erroneous results or conclusions (Kellner \& Swihart, 2014).

Several factors can make it particularly difficult to detect individuals, like for instance nocturnal, cryptic or elusive behavior, burrowing behavior, low population density, habitat characteristics, or even the experience of the observer or surveyor (López-Bao, et al., 2018; Elliot \& Gopalaswamy et al., 2017). All of these factors affect the estimation of variables of interest (e.g., abundance, occupancy) as well as the vital rates that produce demographic or distribution changes (e.g., survival probabilities for abundance; extinction probabilities for occupancy; Mazerolle et al., 2007). In addition to the imperfect detection of species, other factors can be included such as variation in detection probability across space and time, and inappropriate or insufficient spatial sampling that can also bias population size or density estimates (Williams et al., 2002), thus having erroneous information for conservation and management plans or decisions.

Imperfect detection is usually a recurring problem within different sampling methods, especially when individuals are rare, difficult to observe, detect or trap (Gu \& Swihart, 2004; Glisson et al., 2017). However, it is important to note that even when a species is easy to identify and to observe, detection may still be imperfect (Cablk \& Heaton, 2006; Anderson, 2009). This is especially true within natural systems it is a very hard task to detect species perfectly (Goswami et al., 2019). Difficulties in detection may occur for two reasons: (i) the species is completely absent from the survey area, or (ii) the species is present but not detected during the survey, because of the sampling 
methods applied, the behaviour of the animals or the ability of the observed who carries out the survey to detect it (MacKenzie et al., 2006; Gygli, 2017).

In statistical approaches such as occupancy models, accounting for detection probability increases the performance of the models (Glisson et al., 2017), most especially when these models are applied for rare or difficult to detect species (Rota et al., 2011; Shannon et al., 2014). As a result, occupancy models were developed to solve the problems created by imperfect detectability in which these models make use of information from repeated observations at each site to estimate detectability. The precision of the estimated occupancy is higher when the detection probability of the individual increases, providing better estimates and narrower confidence intervals (MacKenzie et al., 2009).

In a recent study, Harkins et al., (2019) made some simple technical changes or improvements to small mammals trapping methods, such as the use of different trap types (e.g. Havahart trap had the higher success on capture rate), use different baits (e.g. pocket mouse, Perognathus fasciatus, had a clear preference for bird seeds), and increasing the trap grid size, but maintaining trapping effort lead to the improvement in detectability of rare and elusive small mammals. In this case, (chapter 1; Romairone et al., 2018), I was able to increase the detection of the individuals through technical improvements such as the adjustment of the trap configuration (decreasing the distance between traps from 12 to $9.6 \mathrm{~m}$; chapter 1 ) to accommodate for females voles having smaller areas of activities (i.e. home range), which granted better estimates of population size and density, a key parameter for population dynamics or management studies (Sutherland et al., 2014; Krebs \& Boonstra, 1984). Through increasing detection probability, our population estimates became 
more precise (e.g. decreasing the error, i.e. SE of the estimates). Having more precisely estimated population densities allows for a better ecological understanding of the study species and, consequently, an improved ability to make effective management decisions.

\subsection{Capture-recapture studies and how they deal with detectability}

Imperfect detection, and the problems which arise because of it, have been recognized for a long time. Both the statistical analysis and experimental design of capture-recapture (CR) studies have seen a gradual development towards those which are able to explicitly incorporate detection probability within analysis (Kéry \& Schmidt, 2008; Kellner \& Swihart, 2014). One of the first methods to integrate detectability into estimates of population abundance estimates was through the application of CR methods (Jiménez, 2017). In essence, a CR approach assumes that when capturing individuals within a demographically and spatially closed sample area, the same individuals will have been present (within the same sample area) throughout the length of the study period. In turn, this means that captures would be from a fixed population allowing inferences to be drawn regarding the probability of capture (Otis et al., 1978; White et al., 1982). The advances in CR, therefore, allowed a model to take into account various forms of detection variability. For instance, as demonstrated in chapter 1 (Romairone et al., 2018), the probability of detection can vary over time $\left(M_{t}\right)$, with individual behavior $\left(M_{b}\right)$, heterogeneity $\left(M_{h}\right)$ and combinations of these (Otis et al., 1978). $\mathrm{Mh}_{\mathrm{h}}$ in particular, challenges the basic premise that there is an equal probability of capture for all individuals in all traps, an assumption that is unfulfilled in most cases (Jiménez, 2017). 
Any given species will display particular complexities when it comes to the estimation of model parameters which can be incorporated within the modeling process (chapter 1). Such variables may include differences in behavior (e.g. trap shy vs trap happy individuals; Yip et al., 2000), an effect of the particular trapping device used, which may include the influence of residual odours left from previous individuals captured (or conversely neophobia). Following this, Brouard et al., (2015) showed that the capturability of wild rodents is determined by the previous trap occupant (i.e. olfactory cues left by previous individual captured). They found that of the three studied species (Apodemus sylvaticus, Myodes glareolus and Microtus agrestis) had the tendency to enter the traps with same-species that have been previously used. Similarly, Boonstra \& Krebs (1976) found that voles entered significantly more traps when the trap was dirty rather than clean. Additionally, Boonstra \& Krebs (1976) found that using clean traps lead to underestimated population density. As a result of the insights gained from the covariates retained in our SCR models, I found that at the beginning of our study, when there were no detections of voles during the first capture occasions (chapter 1), that this increased during next capture occasions, implying a period of time was required for the voles to become familiar with the traps. Therefore, I suggest that the increased probability of detection was likely due to a progressive increase in olfactory cues (i.e. biological residuals/odours) left by the previously captured individuals (faeces, urine, saliva) that subsequently increased detection and trapping rate over time (Chapter 1).

Currently, several developments in Bayesian statistical frame analysis have correspondingly brought a rapid development of CR models. In this way, 
CR models have become tremendously stronger in power and flexibility (Amstrup et al., 2005; Royle et al., 2014) being the cornerstone the capacity of estimation of both population size, as well as vital rates over time (Goswami et al., 2011). This follows earlier work by Efford (2004) in a frequentist framework but this has now moved to a Bayesian framework, which has merged the analysis of animal movements with CR models, resulting in Spatial capturerecapture (SCR) models to estimate density and other key ecological related parameters (Royle \& Young 2008; Royle et al., 2014; Ergon \& Gardner, 2014).

Consequently, taking into account all these model considerations into CR models and improved with SCR modelling provide useful ecological information about the study species and population, and, at the same time, reduce to maximum the potential problems related to the imperfect detection of individuals and thus, the uncertainty around density estimates (chapter 1).

\subsection{Spatial capture-recapture studies}

SCR models represent an extension of classical CR methods, which were nonspatial (Royle et al., 2014; Efford, 2004; Royle et al., 2014, 2017; Efford et al., 2009). SCR modelling allows for both the spatial configuration of sampling devices as well as the spatial arrangement of the species (i.e., spatial encounter histories) to be explicitly included (chapter 1). SCR models are able to incorporate (i) the capture histories with the associated spatial information (location in the space) of the sampling devices and consider, (ii) that capture probability decreases with the relative distance between the sampling device and the activity center of the animal (Royle et al., 2014, 2017). The variation of the capture probability in relation to the relative distance between the sampling 
device and the center of activity of the animal is distributed in a semi-normal or exponential way (Royle et al., 2014).

Regarding animal behavior, movement and effective sampling area, territorial individuals typically make use of a well-defined area around a center of activity (the center of the home range) where capture probability will be highest (i.e. when the trapping is closest to the activity center of the animal home range; Royle et al., 2014). Conversely, traps further away from these activity centers will have the lowest capture probability (but see Traviani et al., 1993; Harris \& Knowlton, 2001). Given the impact of trap location relative to the activity center of the home range, there was a recognized need to define an effective trapping area (White et al., 1982). To solve this, Nichols \& Karanth (2002) proposed using the average distance a species would move MMDM ("Mean Maximum Distance Moved"), or half of the MMDM, as a buffer within the effective sampling area. In order to use a MMDM approach, however, requires knowledge of the spatial ecology of the species studied (Jiménez, 2017). To address this need, novel and advanced methods were developed to update and improve the effective sampling area incorporating the spatially explicit individual's movements into the modelling (Royle et al., 2014). Within this thesis, these activity centers were assumed to be a circular range (Royle et al., 2014). As a result, I have shown that home ranges differed seasonally between sexes during the study period (chapter 1 and 3), for example, with greater estimated home ranges in males $\left(1240 m^{2}\right)$ than females $\left(316 m^{2}\right)$ at the beginning of the breeding season in June 2016.

Therefore, the use of the SCR methods have allowed two important CR limitations to be overcome: the heterogeneity in the detection between 
individuals, motivated by the relative location between the capture device and the animal activity center; and, when working within state-space, the area in which the density will remain invariable (Royle et al., 2014; Chapter 1; Romairone et al., 2018): there is no need to make an arbitrary selection of the study area for obtain $N$ individuals.

SCR models are now routinely applied in many ecological studies. The development of the method has coupled statistically consistent estimates while maintaining a flexible framework for a large variety of ecological spatial theories (Royle et al., 2017). These have included, dispersal and survival (Ergon \& Gardner, 2014; Schaub \& Royle, 2014), resource selection information (Royle et al., 2013), landscape connectivity (Sutherland et al., 2015), habitat fragmentation (Bischof et al., 2017), landscape conservation management (Morin et al., 2017) and epidemiology (Muneza et al., 2017). Increasing the appeal of the approach, SCR models can be fit using data collected from a large variety of monitoring methods (e.g., non-invasive methods, abundance indices, camera trapping or genetic sampling).

There exists a plethora of encounter data on wildlife populations without having to capture or handle individuals (Gardner et al., 2010) such as (i) abundance indices (Jareño et al., 2014; Gervais, 2010) (ii) genetic sampling methods (Mowat \& Strobeck, 2000; Boulanger \& McLellan, 2002; López-Bao et al., 2018) and (iii) camera trapping (Karanth \& Nichols, 1998; Trolle \& Kery, 2003) which are increasingly being used and enable the usage of CR models for many species where it would otherwise be unfeasible to monitor due to the difficulty of capturing individuals. For instance, non-invasive methods such as abundance indices have been widely used in voles for decades (Hansson, 
1979; Delattre et al., 1990; Gervais, 2010), though these require a validation, i.e. to establish the correlation between the activity sign index and true vole abundance (chapter 2).

It should be noted that recent progress has been made in monitoring voles using non-invasive methods where indices of abundance have been used in combination with genetic extraction from excrements found while monitoring. This combination was used with a study focused on the Portuguese endemic Cabrera vole ( $M$. cabreare) where, for first time genetic non-invasive sampling (gNIS) was combined with SCR modelling to provide estimates of size of the populations which has since been used to help understand the demography and conservation status of the Cabrera vole. In this case, Sabino-Marques et al., (2018) evaluated the potential and limitations of this approach to estimate population densities for small mammals inhabiting patchily distributed habitats.

Therefore, SCR models can be applied to data obtained in a multitude of ways from traditional CMR invasive methods to non-invasive monitoring methods (Kéry et al., 2011). In this study (chapter 2) I was able to validate and calibrate the abundance estimates (VAS method) through comparison with SCR derived density estimates (e.g. seasonal variation explained by a lower detectability of VAS with increasing vegetation height, and with reduced vole activity (as estimated from vole detectability in traps). This thesis has shown that models are able to offer a novel perspective of view which takes into account the movement of individuals (sigma) and the probability of detection $\left(g_{0}\right)$, as it will be discussed below. 


\subsubsection{Making more use of SCR model outputs}

One of the main objectives of many studies that make use the SCR methodology is to obtain a robust and precise estimate of density (Efford et al., 2009; Sollmann et al., 2011; Royle et al., 2011, 2014) in order to inform conservation, management or protection plans (Kéry et al., 2011; Gray \& Prum, 2011). However, parameters which explore additional behavioral effects are rarely used (but see Casula et al., 2018). SCR modelling provides relevant information on how the probability of detection varies according to sex, time or behavior (chapter 1), and how these decrease as a function of the distance between an individual's activity center and a detector (e.g., devices such as traps, cameras, hair snares: or observers in transects), and informs on whether these parameters differ between sexes or not. To date, only one paper on small rodents has made use of this information and evaluated whether home range size (as estimated by the sigma parameter) varied with population density. In their study, Casula et al. (2018) found that home ranges of co-occurring Apodemus flavicolis and Myodes glareolus where both negatively related to intraspecific density, meaning that the respective species tended to reduce both home range and dispersal with higher values of abundance resources which is related with high density populations.

Regarding home range estimation, a large variety of statistical models of home range estimators have been developed to date, with increasing complexity and sophistication from the simpler ones, like very high-frequency (VHF), to the most advanced like the global position system (GPS) locations (Kie et al., 2010). In spite of the advances of GPS devices (i.e. as opposed to VHF) there are still some technical issues between the frequency of the data 
collected by the device and the battery life in small vertebrate species because of the problem of the device weight (Kie et al., 2010), in addition to some bias (e.g. missing data and locations) which introduce further detection variation which must be taken into account, such as varying observability within distinct habitat types and landscape peculiarities (Frair et al., 2004, 2010).

In relation to the information obtained from the sigma parameter (movement) and the detection probability $\left(g_{0}\right)$ in this thesis, I have shown that using all this information it has been possible to improve our understanding of the basic ecological functioning of a population of the common voles, but it has also allowed to understand certain biases that have occurred in a capture method that does not take into account the heterogeneities in the probability of capture (in this case, due to sex-specific features; chapter 3) whereas males move larger distance (higher sigma parameter), meanwhile females moved less and were more detectable. As well as the seasonal variation in detecting VAS due to lower detectability of VAS with reduced vole activity (as estimated from vole detectability in traps; chapter 2). In addition, I emphasize that the movement of the females was affected, so that they reduced their movements when more reproduction of females was detected.

SCR models can also be enriched with further variables, such as movement data as explained previously (e.g. telemetry data -variable that can be inaccurate in a single way but can be stronger complementing SCR models) to better improve the calculation of the movement parameter sigma, or incorporate sampling characteristics (e.g. device operability, closed or stolen traps, etc.). Moreover, the SCR models give us the opportunity to visually get the space use by the individuals captured through the map of activity centers 
but also to work with the maps because the outputs (e.g. raster map) are setting by pixels and therefore containing information within (e.g. activity centers). Moreover, exemplifying how the output of SCR can be better utilized, the output maps in this thesis were used to extract the density values that overlapped with the transects line areas used in obtaining the number of voles per transects (chapter 2).

In this thesis I used the information obtained from the monitoring of a free-ranging population of common voles in an experimental plot that was carried out during a decline phase of the population between the years 2016 to 2018, following a population peak in summer 2014. Based on a well-structured monthly monitoring design, I was able to: (i) adapt the SCR methodology for the study of small mammals identifying those key aspects, both design and modeling for the proper functioning of the methodology and obtain estimates of reliable density. I have also used robust and precise estimates to (ii) validate an indirect method based on abundance indices (VAS) identifying those aspects that should be taken into account to improve VAS predictions and, finally to (iii) validate the adult sex-ratio of a direct method that does not take into account the heterogeneity of the capture of the individuals, highlighting the variables and proposing alternatives to reduce the potential biases in the capture.

\subsubsection{Limitations of SCR models to our model species}

Although the SCR models are the state-of-art for estimating densities, they also have some drawbacks and would benefit from further improvements. In the thesis study species, the common vole, which has burrowing habits, and spends most of the time within chambers and tunnels underground, the majority 
of individuals may not be detected since some age-classes like the newborn suckling pups or those that have not yet left the nest may not be taken into account (i.e. age or stage dependent detectability) since they have not been captured by the traps leading to underestimating population size (abundance or density). Moreover, it is important to highlight that SCR modelling assumes that home range has a circular shape (see Royle et al., 2014; Chapter 1; Romairone et al., 2018). However, in the study species tend to occur in linear habitats, often occurring within field margins (Rodríguez-Pastor et al., 2016) [see outputs maps of chapters 1 and 2]. Therefore, when voles occur in fields, circular home ranges are likely suitable as colonies are circular by nature, however, when occupying field margins the home range shape is more likely linear, due to the elongated and narrow shape of the margins. In such scenarios the home range assumption, is likely violated, meaning it would be desirable that home range shape is made context specific, allowing an "edge effect" to be incorporated in the future. A further limitation of the SCR methodology is that for proper functioning a large number of spatial recaptures are required (i.e. marked individuals captured in more than one trap during the session of the species of study; chapter 1). However, with our study this was not an issue, but it could be a limitation for other species that may have lower capturability.

\subsection{Future research lines}

The use of the SCR models, and of the parameters they provide, opens a wide range of possibilities to continue researching and learning about the ecology of our study species, the common vole. Additional research can be done in order to expand knowledge about other demographic parameters not investigated in 
the present study (e.g. survival, immigration/emigration), the space use between males and females, and the possibility of a spatial sexual segregation or of spatial structuring based on colonies of females cooperating during breeding). It can also be extended to space use by different species living in the same area (potential interspecific competition between common voles and wood mouse). It should be noted that the results of this thesis were focused on a decreasing phase of the population, but it would be vital to follow the same steps during an increase phase. Repeating the same monitoring during a vole increase phase (growing demographic phase) would be also very useful to better understand how demography, behavior and space use differ between the increase and decline phase of vole cycles.

Given a sufficiently large scale long term data set (i.e. like the several primary sessions of the present study), more robust and powerful demographic studies could be carried out (extending beyond variations in density, sex-ratio and space use), which could investigate variation in survival between individuals across populations over space and time. Methods such as CR allow us to obtain information on population dynamics, demography and life-history traits in wild populations (e.g. Williams et al., 2002; Ergon \& Gardner, 2014). By using these methods along with appropriate statistical modelling, interesting demographic parameters can be obtained, like true survival (i.e. as opposed to apparent survival) especially in open-populations (see Ergon \& Gardner, 2014). Traditional CR models are often referred to as estimating 'apparent survival' because the method is not able to distinguish between the different sources of missing individuals (e.g. mortality, emigration) and therefore only estimate the combined probability of surviving and not emigrating from the study area 
between primary sessions (Ergon \& Gardner, 2014). In a recent study using field voles (M. agrestis) as a model species, Ergon \& Gardner (2014) applied an extension in the SCR methodology for robust-design data (Gardner et al., 2010) to obtain true survival estimates by separating mortality and emigration. This methodology could be similarly be applied to the data collected during this thesis.

In terms of population structure, the sex-ratio has been studied in this thesis, but other aspects remain to be explored, like for instance age structure (e.g. juveniles, sub-adults) and its interaction over time. It would be interesting to study how male and female juveniles, sub-adults and adult individuals are distributed in space across seasons. We could better understand the use of the space of individuals throughout the seasons and be able to better understand the ecology of the species of study, for instance, in an important season, like spring, when the reproduction starts.

Other potential research perspectives would be the use of spatial information to the potential existence of sexual segregation. Such information is difficult to gather when the species spend most of their time underground and not on the surface but can be evaluated using SCR model outputs. Classically, sexual segregation has been described as the differential use of resources by the sexes out of the mating season (e.g. Barboza \& Bowyer, 2000, Bleich et al., 1997; Bowyer, 1984; Kie \& Bowyer, 1999; McCullough et al., 1989). Sexual segregation has important ecological consequences for population dynamics such as individual increase and breeding success. Three types of sexual segregation may occur: i) sexes live in separate groups out of the breeding season, ii) sexes differ in their use of habitat, and iii) sexes occupy different 
zones within the same habitat (Conradt, 2005). New advances in SCR models have suggested that this methodology is relatively robust to spatial segregation (or aggregation) of individuals (López-Bao et al., 2018), a main property of social animals that living in well-defined groups that could potentially violate the assumption that activity centers are independently distributed over the statespace S (Royle et al., 2014). By analyzing the use of space by female and male voles, we could determine the degree of sexual segregation of both groups at certain times of the year, for example, in the breeding season. This issue has been shown in other species of Microtines, in which females aggregate in groups at certain times of the year (e.g. kinship, relatedness, territoriality) and cooperate during breeding and attempt to avoid infanticide or to maintain their colony or territory. In this case, territorial defense in females is mostly related to food resources, shelter or nest sites, but exclusive territories may be needed to counter infanticide, with other females posing a higher risk than males (Wolff, 1993).

It would similarly be interesting to analyze the use of the space by different species inhabiting the same area. This could also be done by using the output maps of the SCR modelling. It is known that spatial distribution and resource abundance define animal spacing behavior (Ostfeld, 1990). Therefore, some species with similar life-style or requirements may share available resources (Leibold \& McPeek, 2006). However, interspecific competition can affect space use patterns, with home ranges becoming smaller with higher density of resources (Mazurkiewicz \& Rajska-Jurgiel, 1998; Stradiotto et al., 2009) or animals (Sanderson, 1996; Efford et al., 2016). Nevertheless, this competition usually is less intense (e.g. in comparison with intraspecific 
competition) as a consequence of higher niche differentiation between competing species (Chesson, 2000). 


\section{Conclusions}

- The monitoring of small mammals is traditionally done by means of activity signs or trappings, and both methods have associated biases related to variation in detection probability or capturability. Understanding and correcting for these biases is necessary to obtain reliable estimates of abundance.

1. In order to successfully apply spatial capture-recapture methods, basic knowledge on the study species is necessary. A pilot study may be particularly useful in the absence of a priori knowledge.

2. Particular attention must be paid to: (i) reducing mortality in traps, (ii) evaluating the influence of animal behavior on capture rate, (iii) monitoring saturation levels and adjusting the number of traps and/or time intervals between capture occasions accordingly, and (iv) adjusting the trap configuration (minimum distance between traps) to account for the variation in movement patterns of the species under study.

3. Monitoring based on vole activity signs provide a useful tool for large-scale monitoring of rodents, being easy to use, cheap and requiring less time than trapping methods. Indirect methods should however be validated using accurate vole density estimates.

4. The performance of a vole activity signs method varied among seasons, with fewer signs detected than that expected compared to actual density during spring-summer as compared with autumn-winter. This could be due to a 
lower detectability of vole activity signs indices when vegetation is taller and vole activity above ground is reduced. Vole activity sign assessments perform better in fields than in margins.

5. Estimating the sex-ratio of an adult population using single trapping sessions is subject to potential biases. Adult sex-ratio was consistently female-biased when estimated using the spatial capture-recapture methodology, but more often male-biased when estimated using single trapping sessions.

6. Biases in adult sex-ratio estimation varied seasonally and were correlated with variations in female movement and male detection probability. The proportion of reproductive females was also linked to deviations in adult sexratio estimated using each method, and could be used, together with season, as a correcting factor.

7. The maximum vole density reached during this study was 113 voles per ha and was conducted during a decline phase (common vole populations in the region can reach densities of $>1000$ voles per ha at population density peaks). Further work would thus be needed to calibrate the vole activity sign method and the estimates of adult sex-ratio at higher vole densities and during the increase phase of vole outbreaks. 


\section{Conclusiones}

- La monitorización de pequeños mamíferos se realiza tradicionalmente a través de signos y rastros de su actividad o bien capturándolos mediante el uso de trampas, donde ambos métodos tienen sesgos asociados con la variación en la probabilidad de detección o capturabilidad. Es necesario comprender y corregir estos sesgos para obtener estimas precisas y fiables de la abundancia.

1. Para poder aplicar con éxito la metodología de captura-recaptura espacial, es necesario un conocimiento ecológico básico de la especie estudiada. Un estudio piloto es necesario en ausencia de un conocimiento previo sobre el comportamiento de la especie en cuestión.

2. Se debe prestar especial atención a: (i) reducir la mortalidad en trampas, (ii) evaluar la influencia del comportamiento del animal en la tasa de captura, (iii) monitorizar los niveles de saturación y ajustar el número de trampas y/o intervalos de tiempo entre las ocasiones de captura, y (iv) ajustar la configuración de la malla de trampeo (distancia mínima entre trampas) a la variación de los patrones de movimiento de la especie en estudio.

3. La monitorización basada en los signos de actividad de topillos es una herramienta útil para el monitoreo a gran escala de roedores, fácil de usar, barato y menos costoso que los métodos de captura. Sin embargo, los métodos indirectos deben validarse utilizando estimas precisas de la densidad de topillos. 
4. El uso del método basado en signos de actividad varió estacionalmente, con menos signos detectados de lo esperado a partir de la densidad durante la primavera-verano en comparación con el otoño-invierno. Esta variación estacional podría deberse a una menor detectabilidad de los índices de signos de actividad cuando la vegetación es más alta y la actividad de los topillos en la superficie se ve reducida. Las evaluaciones de los signos de actividad funcionan mejor en el interior de los campos que en los márgenes.

5. La estima de la proporción de sexos de individuos adultos en la población mediante sesiones de trampeo de un solo evento está sujeta a posibles sesgos. La proporción de sexos adultos tuvo un sesgo sistemático hacia las hembras cuando se estimaron utilizando la metodología de capturarecaptura espacial, sin embargo, estuvo sesgado hacia machos cuando se estimaron usando sesiones de trampeo únicas.

6. Los sesgos en la estima de la proporción de sexos de individuos adultos variaron según la estación y se correlacionaron con las variaciones en el movimiento de las hembras y la probabilidad de detección de los machos. La proporción de hembras reproductoras en un mes dado se vinculó a las desviaciones en la proporción de sexos adultos estimada utilizando cada método, y podría usarse, junto con la estación, como un factor de corrección.

7. La densidad máxima alcanzada en el estudio fue de 113 topillos por hectárea a lo largo de una fase de disminución poblacional (las poblaciones de topillo campesino pueden sobrepasar los más de 1000 individuos por ha 
en los picos de densidad máxima en la región). Por lo tanto, se necesitaría más trabajo para calibrar el método de signos de actividad de topillos y las estimas de la proporción de sexos adultos en densidades de topillos más altas y/o durante la fase de aumento exponencial de topillos. 


\section{References}

Acevedo, P., Vicente, J., Höfle, U., Cassinello, J., Ruiz-Fons, F., Gortazar, C., 2007. Estimation of European wild boar relative abundance and aggregation: a novel method in epidemiological risk assessment. Epidemiol. Infect. 135, 519-527. DOI:10.1017/S0950268806007059

Akaike, H., 1974. A new look at statistical model identification. IEEE Trans. Automat. Contr.19, 716-723.

Amstrup, S.C., McDonald, T.L., Manly, B.F.J., 2005. Handbook of capturerecapture analysis. Princeton University Press, Princeton, New Jersey, USA. ISBN-13: 978-0-691-08967-6

Amrhein, V., Scaar, B., Baumann, M., Minéry, N., Binnert, J., Korner-Nievergelt, F., 2012. Estimating adult sex ratios from bird mist netting data. Methods in Ecology and Evolution, 3: 713-720. doi:10.1111/j.2041210X.2012.00207.x

Amori, G., Locasciulli, O., Tuccinardi, P., Riga, F., 2000. Ecological structure of a population of Clethrionomys glareolus in central Italy: An eight-year study. Polish Journal of Ecology 48:125-132

Anderson, D.L., 2009. Ground Versus Canopy Methods for the Study of Birds in Tropical Forest Canopies: Implications for Ecology and Conservation. Condor. 111: 226-237

Anthony, N.M., Ribic, C.A., Bautz, R., Garland, T. Jr., 2005. Comparative effectiveness of Longworth and Sherman live traps. Wildlife Society Bulletin; 33(3)

Ancona, S., Dénes, F. V., Krüger, O., Székely, T. \& Beissinger, S.R., 2017. Estimating adult sex ratios in nature. Philosophical Transactions of the Royal Society B: Biological Sciences. Vol. 372, Issue 1729. DOI:https://doi.org/10.1098/rstb.2016.0313

Anderson, D.R., 2003. Index values rarely constitute reliable information. Wildlife Society Bulletin, 31, 288-291.

Alldredge, M.W., Simons, T.R., Pollock, K.H., 2007. Factors affecting aural detections of songbirds. Ecological Applications 17:948-955

Aplin, K.P., Brown, P.R., Jacob, J., Krebs, C.J., Singleton, G.R., 2003. Field methods for rodent studies in Asia and the Indo-Pacific. ACIAR Monograph No. 100, 223p. ISBN 1863203931

Aschwanden, J., Holzgang, O., Jenni, L., 2007: Importance of ecological compensation areas for small mammals in intensively farmed areas. Wildl. Biol. 13: 150-158. 
Barboza P. S. Bowyer R. T. 2000. Sexual segregation in dimorphic deer: a new gastrocentric hypothesis. Journal of Mammalogy 81:473-489.

Batzli, G. O., Pitelka, F. A., Cameron, G. N., 1983. Habitat use by lemmings near Barrow, Alaska. Holarctic Ecology 6: 255- 262.

Baker, J.A., Brooks, R.J. 1981. Distribution patterns of raptors in relation to density of meadow voles. Condor 83: 4247

Battersby, J., Jeremy, J., Greenwood, D., 2003. Monitoring terrestrial mammals in the UK: past, present and future using lessons from the bird world. Mammal review. Vol.34, Issue 1-2, 3-29.

Bennett, A.F., Owens, I.P.F., 2002. Evolutionary Ecology of Birds: Life Histories, Mating Systems and Extinction. Oxford: Oxford University Press. ISBN10: 0198510896

Bessa-Gomes, C., Legendre, S., Colbert, J., 2004. Allee effects, mating systems and the extinction risk in populations with two sexes. Ecol. Lett., 7:802-812. DOI: 10.1111/j.1461-0248.2004.00632.x

Berger, J., Gompper, M. E., 1999. Sex ratios in extant ungulates: products of contemporary predation or past life histories?. Journal of Mammalogy 80: 1084-1113.

Bischof, R., Steyaert, S., Kindberg, J., 2017. Caught in the mesh: roads and their network-scale impediment to animal movement. Ecography. Vol. 40, Issue, 12, pp. 1369-1380. https://doi.org/10.1111/ecog.02801

Bleich V. C. Bowyer R. T. Wehausen J. D. 1997. Sexual segregation in mountain sheep: resources or predation? Wildlife Monographs 134:150.

Boonstra, R., Kanter, M., Krebs, C.J., 1992. A tracking technique to locate small mammals at low densities. - J. Mammal. 73: 683-685.

Boonstra, R. \& Krebs, C. J. 1976. The effect ofodour on trap response in 3ticrotus townsendii. J. Zool., Lond., 180,467-476.

Boulanger, J., G. C. White, B. N. McLellan, J. Woods, M. Proctor, and S. Himmer. 2002. A meta-analysis of grizzly bear DNA mark-recapture projects in British Columbia. Ursus 13:137-152. [online] URL: www.ecological.bc.ca/ursus_2002_dna_meta_analysis.pdf

Borchers, D.L., Efford, M.G., 2008. Spatially explicit maximum likelihood methods for capture-recapture studies. Biometrics;64: 377-385.

Borowski, Z., 2003. Habitat selection and home range size of field voles Microtus agrestis in Sowiñski National Park, Poland. Acta Theriologica. 48 (3): 325-333.

Borowski, Z., Owadowska, E., 2009. Field vole (Microtus agrestis) seasonal 
spacing behavior: the effect of predation risk by mustelids. Naturswissenschaften. Vol.97, Issue 5, pp. 487-493

Boyce, C.C.K., Boyce, J.L., 1988. Population biology of Microtus arvalis I. Lifetime reproductive success of solitary and grouped breeding females. J. Anim. Ecol., 57: 711-722.

Bowyer R. T. 1984. Sexual segregation in southern mule deer. Journal of Mammalogy 65:410-417.

Brown, J.S., 1988. Patch use as indicator of habitat preference, predation risk and competition. Behav Ecol Sociobiol 22:37-47

Brown, J.S., Laundré, J.W., Gurung, M., 1999. The ecology of fear: optimal foraging, game theory, and trophic interactions. J Mammal 80:385-399

Brouard, M.J., Coulson, T., Newman, C., Macdonald, D.W., Buesching, C.D., 2015. Analysis on population level reveals trappability of wild rodents is determined by previous trap occupant. PLoS One;10: 1-15. pmid:26689683

Brügger, A., Nentwig, W., Airoldi, J.P., 2010. The burrow system of the common vole (M. arvalis, Rodentia) in Switzerland. Mammalia;74: 311-315.

Briner, T., Nentwig, W., Airoldi, J.P., 2005. Habitat quality of wildflower strips for common voles (Microtus arvalis) and its relevance for agriculture. Agriculture Ecosystems \& Environment.105(1-2):173-179.

Brügger, A., Nentwig, W., Airoldi, J-P., 2010. The burrow system of the common vole (M. arvalis, Rodentia) in Switzerland. Mammalia;74: 311-315.

Bryja, J., Nesvadbová, J., Heroldová, M., Jánová, E., Losík, J., Trebatická, L., et al. 2005. Common vole (Microtus arvalis) population sex ratio: biases and process variation. Can J Zool.;83: 1391-1399. doi:10.1139/z05-133

Buckland, S.T., Marsden, S.J., Green, R.E. 2008. Estimating bird abundance: making methods work. Bird Conservation International, 18, 91-108.

Burnham, K., Anderson, D., 2002. Formal Inference From More Than One Model: Multimodel Inference (MMI). In: Burnham K.P., Anderson D.R. (eds) Model Selection and Multimodel Inference. Springer, New York, NY. DOI: https://doi.org/10.1007/978-0-387-22456-5_4

Bujalska, G., Saitoh, T., 2000. Territoriality and its consequences. Polish J Ecol; 48:37-49. https://www.infona.pl/resource/bwmeta1.element.baztech-articleBGPK-0055-2148

Bryja, J., Nesvadbová, J., Heroldová, M., Jánová, E., Losík, J., Trebatická, L., et al., 2005. Common vole (Microtus arvalis) population sex ratio: biases and process variation. Can J Zool;83: 1391-1399.

Cablk, M.E., Heaton, J.S., 2006. Accuracy and Reliability of Dogs in Surveying for Desert Tortoise (Gopherus agassizii). Ecol Appl. 1926-1935. 
pmid:17069383

Casula, P., Luiselli, L., Amori, G., 2018. Which population density affects home ranges of co-occurring rodents?. Basic and Applied Ecology. Vol(34), 4654 .

Capizzi, D., Luiselli, L., 1996. Feeding relationships and competitive interactions between phylogenetically unrelated predators (owls and snakes). Acta Oecologica. 17: 265-84.

Castañeda, I., Pisanu, B., Díaz, M., Rézouki, C., Baudry, E., Chapuis, J-L., Bonnaud, E., 2018. Minimizing trapping effort without affecting population density estimations for small mammals. Mammalian Biology.93, 144-152.

Conradt, L. 2005. Definitions, hypotheses, models and the measures in the study of animal segregation. Pp. 11-34 in Sexual segregation in vertebrates: ecology of the two sexes (K. E. Ruckstuhl and P. Neuhaus, eds.). Cambridge University Press, Cambridge, United Kingdom

Clarke, A. L., B.-E. Sæther, and E. Røskraft. 1997. Sex bias in aviandispersal: a reappraisal. Oikos 79:429-438.

Clout, M.N, Elliott G.P, Robertson B.C., 2002. Effects of supplementary feeding on the offspring sex ratio of kakapo: a dilemma for the conservation of a polygynous parrot. Biol. Conserv.107:13-18. doi:10.1016/S00063207(01)00267-1

Clutton-Brock T., 2007. Sexual selection in males and females. Science;318(5858):1882-5.

Conn, P.B, Arthur, A.D, Bailey, L.L, Singleton, G.R, 2006. Estimating the abundance of mouse populations of known size: Promises and pitfalls of new methods. Ecological applications. Vol.16, Issue 2. Pages 829-837.

Cornulier, T., Yoccoz, N.G., Bretagnolle, V., Brommer, J.E., Butet, A., Ecke, F., Elston, D.A., Framstad, E., Henttonen, H., Hornfeldt, B., Huitu, O., Imholt, C., Ims, R.A., Jacob, J., Jedrzejewska, B., Millon, A., Petty, S.J., Pietiainen, H., Tkadlec, E., Zub, K., Lambin, X., 2013. Europe-Wide Dampening of Population Cycles in Keystone Herbivores. Science (80-.). 340, 63-66. https://doi.org/10.1126/science.1228992

Chao, A., Huggins, M., 2005. Classical Closed-population Capture-Recapture Models. In: Amstrup, S.C., McDonald, T.L., Manly, B.F.J, editors. Handbook of capture-recapture analysis. 2010th ed. Princeton University Press; $2005 . \quad$ pp. 23-35. Available: https://www.safaribooksonline.com/library/view/handbook-ofcapture-recapture/9781400837717/

Chesson, P., 2000. Annual Review of Ecology and Systematics. Volume 31, pp 343-366. https://doi.org/10.1146/annurev.ecolsys.31.1.343 
Chitty, D.H., Kempson, D.A., 1949. Pre-baiting small mammals and a new design of live trap. Ecology; 30:536-542.

Chandler, R.B., Royle, J., 2013. Spatially explicit models for inference about density in unmarked or partially marked populations. Ann Appl Stat;7: 936-954.

Dawson, D.K, Efford, M.G., 2009. Bird population density estimated from acoustic signals. Journal of Applied Ecology 46: 1201-1209.

Dale, S., 2001. Female-biased dispersal, low female recruitment, unpaired males, and the extinction of small and isolated bird populations. Oikos 92: 344-356.

Delibes, J., 1989. Plagas de topillos en Espana. Quercus 35, 17-20

Delibes-Mateos, M., Ferreras, P., Villaduerte, R., 2008. Rabbit populations and game management: the situation after 15 years of rabbit haemorrhagic disease in central-southern Spain. Biodivers Conserv. 17:559-574. DOI $10.1007 / \mathrm{s} 10531-007-9272-5$

Dénes, F.V., Silveria, L.F., Beissinger, S.R., 2015. Estimating abundance of unmarked animal populations: accounting for imperfect detection and other sources of zero inflation. Methods in Ecology and Evolution, Vol 6, issue 5, pp. 543-556. https://doi.org/10.1111/2041-210X.12333

Daedlow, D., Westerman, P. R., Baraibar, B., Rouphael, S., Gerowitt, B., 2014. Weed seed predation rate in cereals as a function of seed density and patch size, under high predation pressure by rodents. Weed Res. 54:186-195

Delattre, P., De Sousa B., Fichet-Calvet, E., Quéré J. P., Giraudoux P. 1999. Vole outbreaks in a landscape con-text: evidence from a six year study of Microtus arvalis. Landscape Ecology 14: 401-412

Delattre, P., Giraudoux, P., Damange, J.P., Quéré, J.P., 1990. A density indicator for monitoring natural populations of common voles Microtus arvalis. Rev. d'Ecologie-La Terre la Vie. https://doi.org/10.1016/j.geomorph.2010.09.029

Dillon, A., Kelly, M.J., 2007. Ocelot Leopardus pardalis in Belize: the impact of trap spacing and distance moved on density estimates. Oryx;41: 469477.

Distiller, G., Borchers, D.L., 2015. A spatially explicit capture-recapture estimator for single-catch traps. Ecol Evol;5: 5075-5087. pmid:26640683

Dizney, L., Jones, P.D., Ruedas, L.A., 2008. Efficacy of three types of live traps used for surveying small mammals in the Pacific Northwest. Northwest Nat;89: 171-180. 
De Valpine, P., Turek, D., Paciorek, C.J., Anderson-Bergman, C., Lang, D.T., Bodik, R., 2017. Programming with models: writing statistical algorithms for general model structures with NIMBLE. J Comput Graph Stat;26: 403-413.

Donald, P. F., 2007. Adult sex ratios in wild bird populations. Ibis, 149: 671-692. doi:10.1111/j.1474-919X.2007.00724.x

Donald, P.F., 2011. Lonely males and low lifetime productivity in small populations. Ibis, 153, 465-467.

Drickamer, L.C., Feldhamer, G.A., Mikesic, D.G., Holmes, C.M., 1999. Trapresponse heterogeneity of house mice (Mus musculus) in outdoor enclosures. J. Mammal.80: 410-420

Elliot, N. B. \& Gopalaswamy, A. M., 2017. Toward accurate and precise estimates of lion density. Conservation Biology 31, 934-943

Eccard, J.A., Klemme, I., 2013. Reducing mortality of shrews in rodent live trapping-A method increasing live-trap selectivity with shrew exits. Ann Zool Fennici;50: 371-376.

Engeman, R.M. Whisson, D.A., 2006. Using a general indexing paradigm to monitor rodent populations. Int Biodeterior Biodegradation 58:2

Engeman, R.M., Massei, G., Sage, M., Gentle, M.N., 2013. Monitoring wild pig populations: a review of methods. Environ. Sci. Pollut. Res. 20, 80778091.

Efford, M.G., 2004. Density estimation in live-trapping studies. Oikos;106: 598610.

Efford, M. G., Mowat, G., 2014. Compensatory heterogeneity in spatially explicit capture-recapture data. Ecology, 95: 1341-1348. doi:10.1890/13-1497.1

Efford, M.G., Borchers, D.L., Byrom, A.E., 2009. Density estimation by spatially explicit capture-recapture: likelihood-based methods. Modeling demographic processes in marked populations. Boston, MA: Springer US;pp. 255-269. https://doi.org/10.1007/978-0-387-78151-8_11

Efford, M.G., 2016. secr: Spatially explicit capture-recapture models. https://CRAN.R-project.org/package=secr

Embleton, N.L., Petrovskaya, N.B., 2013. On numerical uncertainty in evaluation of pest population size. Ecol. Complex. Vol.14, pp. 117-131 https://doi.org/10.1016/j.ecocom.2012.11.004

Ergon, T., Gardner, B., 2014. Separating mortality and emigration: Modelling space use, dispersal and survival with robust-design spatial capturerecapture data. Methods Ecol Evol;5: 1327-1336. 
Erlinge, S., Hoogenboom, I., Agrell, J., Nelson, J., Sandell, M., 1990. Densityrelated home-range size and overlap in adult field voles (Microtus agrestis) in southern Sweden. J. Mammal; 71: 597-603

Fauteux, D., Gauthier, G., Mazerolle, M.J., Coallier, N., Bety, J., Berteaux, D., 2018. Evaluation of invasive and non-invasive methods to monitor rodent abundance in the Arctic. Ecosphere. Vol 9, Issue 2, e02124. https://doi.org/10.1002/ecs2.2124

Ferreria, C.M., Sabino-Marques, H., Barbosa, S., Costa, P., Encarnaçao, C., Alpizar-Jara, R., Pita, R., Beja, P., Mira, A., Searle, J.B., Paupério, J., Alves, P.C., 2018. Genetic non-invasive sampling (gNIS) as a costeffective tool for monitoring elusive small mammals. European Journal of Wildlife Research. 64:46. https://doi.org/10.1007/s10344-018-1188-8

Fisher, R.A., 1930. The Genetical Theory of Natural Selection. Clarendon Press, Oxford. http://dx.doi.org/10.5962/bhl.title.27468

Franklin, T.W., McKelvey, K.S., Golding, J.D., Mason, D.H., Dysthe, J.C., Pilgrim, K.L., Squires, J.R, et al., Schwartz., 2019. Using environmental DNA methods to improve winter surveys for rare carnivores: DNA from snow and improved noninvasive techniques. Biological Conservation. Vol. 229, pp. 50-58. https://doi.org/10.1016/j.biocon.2018.11.006

Frair J. L., Nielsen S. E., Merrill E. H., Lele S. R., Boyce M. S., Munro R. H. M., Stenhouse G. B., Beyer H. L. 2004. Removing GPS collar bias in habitat selection studies. J. Appl. Ecol. 41, 201-212 doi:10.1111/j.00218901.2004.00902.x

Frair J. L., Fieberg J., Hebblewhite M., Cagnacci F., DeCesare N. J., Pedrotti L. 2010.Resolving issues of imprecise and habitat-biased locations in ecological analyses using GPS telemetry data. Phil. Trans. R. Soc. B 365, 2187-2200 doi:10.1098/rstb.2010.0084

Fischer, C., Schröder, B., 2014. Predicting spatial and temporal habitat use of rodents in a highly intensive agricultural area. Agriculture Ecosystems \& Environment, 189, 145- 153.

Fischer, C., Türke, M., 2016. Seed preferences by rodents in the agrienvironment and implications for biological weed control. Ecology and Evolution 6(16):5796-5807. DOI: 10.1002/ece3.2329

Focardi, S., Isotti, R., Raganella-Pelliccioni, E. lannuzzo, D., 2002: The use of distance sampling and mark-resight to estimate the local density of wildlife populations. - Environmetrics 13: 177-186

Flowerdew, J., Shore, R.F., Poulton, S., Sparks, T., 2004. Live trapping to monitor small mammals in Britain. Mammal Review 34(1-2):31 - 50. DOI: 10.1046/j.0305-1838.2003.00025.x 
Gauthier, G., Berteaux, D., Bêty, J., Tarroux, A., Therrien, J.F., McKinnon, L., Legagneux, P., Cadieux, M.C., 2011. The tundra food web of Bylot Island in a changing climate and the role of exchanges between ecosystems, Écoscience, 18:3, 223-235, DOI: 10.2980/18-3-3453.

Gauffre, B., Petit, E., Brodier, S., Bretagnolle, V., \& Cosson, J. F., 2009. Sexbiased dispersal patterns depend on the spatial scale in a social rodent. Proceedings. Biological sciences, 276(1672), 3487-3494. doi:10.1098/rspb.2009.0881

Garrote, G., Pérez de Ayala, R., Pereira, P., Robles, F., Guzmán, N., García, F.J., Iglesias, M.C., Hervás, J., Fajardo, I., Simón, M., Barroso, J.L., 2011. Estimation of the Iberian Iynx (Lynx pardinus) population in the Doñana area, SW Spain, using capture-recapture analysis of cameratrapping data. European Journal of Wildlife Research 57:355-362

Gardner, B., Royle, J.A., Wegan, M.T., Rainbolt, R.E., Curtis, P.D., 2010. Estimating black bear density using DNA data from hair snares. J Wildl Manage;74: 318-325.

Gardner, B., Royle, J.A., Wegan, M.T., 2009. Hierarchical models for estimating density from DNA mark - recapture studies. Ecology 90, 1106-1115. https://doi.org/10.1890/07-2112.1

Gerber, B.D., Parmenter, R.R., 2015. Spatial capture-recapture model performance with known small-mammal densities. Ecol Appl;25: 695705. pmid:26214915

Gervais, J.A., 2010. Testing sign indices to monitor voles in Grasslands and Agriculture. Northwest Science 84, 281-288.

Getz, L.L., McGuire, B., Pizzuto, T., Hofmann, J.E, Frase, B., 1993. Social organization of the prairie vole Microtus ochrogaster. J. Mam- mal. 74: 44 $-58$.

Gilg, O., Hanski, I., Sittler, B., 2003. Cyclic dynamics in a simple vertebrate predator-prey community. Science. 302(5646):866-8

Giraudoux, P., Pradier, B., Delatre, P., Deblay, S., Salvim D., Defaut, R. 1995. Estimation of water vole abundance by using surface indices. Acta Theriologica 40: 77-96.

Gliwicz, J., 1997. Space use in the root vole: basic patterns and variability. Ecography; 20: 383-389.

Godfray H.C.J., Werren J.H., 1996. Recent developments in sex-ratio studies. Trends Ecol Evol 11: 59-63

Goswami, V. R., Getz, L. L., Hostetler, J. A., Ozgul, A. \& Oli, M. K., 2011. Synergistic influences of phase, density, and climatic variation on the dynamics of fluctuating populations. Ecology 92, 1680-1690 
Goswami, V.R., Yadaba, M.K., Vasudev, D., Prasad, P.K., Sharma, P., Jathanna, D., 2019. Towards a reliable assessment of Asian elephant population parameters: the application of photographic spatial capturerecapture sampling in a priority floodplain ecosystem. Scientific Reports, 9. 8578. DOI: 10.1038/s41598-019-44795-y

Gortázar, C., Acevedo, P., Ruiz-Fons, F., Vicente, J., 2006. Disease risks and overabundance of game species. European Journal of Wildlife Research 52, pp. 81-87

Gompper, M. E., Kays, R.W., Ray, J.C, Lapoint, S.D, Bogan, D.A, Cryan, J.R., 2006. A comparison of noninvasive techniques to survey carnivore communities in northeastern North America. Wildl. Soc. Bull. 34: 11421151.

Gygli, A.C., 2017. Integrating molecular and visual surveys to determine factors impacting detection and habitat choice of native montane amphibians in Wyoming and Colorado. University of Wyoming.

Glisson, W.J., Conway, C.J., Nadeau, C.P., Borgmann, K.L., 2017. Habitat models to predict wetland bird occupancy influenced by scale, anthropogenic disturbance, and imperfect detection. Ecosphere. 8: 1-20.

Graham, I.M., Lambin, X., 2002. The impact of weasel predation on cyclic fieldvole survival: the specialist predator hypothesis contradicted. Journal of Animal Ecology. Vol. 71, pp. 946-956.

Gray, T.N.E., Prum, S., 2012. Leopard density in post-conflict landscape, Cambodia: Evidence from spatially explicit capture-recapture. Population Ecology. Vol. 76, Issue 1, pp. 163-169. https://doi.org/10.1002/jwmg.230

Grayson, K.L, Mitchell, N.J., Monks, J.M., Keall, S.N., Wilson, J.N., et al., 2014. Sex Ratio Bias and Extinction Risk in an Isolated Population of Tuatara (Sphenodon punctatus). PLOS ONE 9(4): e94214. https://doi.org/10.1371/journal.pone.0094214

Grimm, A., Gruber, B., Henle, K., 2014. Reliability of different mark-recapture methods for population size estimation tested against reference population sizes constructed from field data. PLoSONE. 9(6):e98840. doi: 10.1371/journal.pone.0098840

Greenwood, J.D., Robinson, R.A., 2006. General census methods. Ecological Census Techniques, 2nd edition (ed. W.J. Sutherland), pp. 87- 185. University Press, Cambridge.

Gurnell, J., Langbein, J., 1983. Effects of trap position on the capture of woodland rodents. Notes from Mammal Soc;3: 289-292.

Gu, W., Swihart, R.K., 2004. Absent or undetected? Effects of non-detection of species occurrence on wildlife-habitat models. Biol Conserv. 116: 195203. 
Güthlin, D., Storch, I., Küchenhoff, H., 2014. Toward Reliable Estimates of Abundance: Comparing Index Methods to Assess the Abundance of a Mammalian Predator. PLoSONE 9(4): e94537. https://doi.org/10.1371/journal.pone.0094537

Gyllenberg, M., Hanski, I., Hastings, A., 1997. Structured metapopulation models. In: Metapopulation Biology: Ecology, Genetics and Evolution, p. 93-122. Hanski, I., Gilpin, M.E., Eds, Academic Press, London.

Han, B.A., Schmidt, J.P., Bowden, S.E., Drake, J.M., 2015. Rodent reservoirs of future zoonotic diseases. Proc Natl Acad Sci;112: 7039-7044. pmid:26038558

Hansson, L., 1979: Food as a limiting factor for small rodent numbers. Test of two hypotheses. Oecologia (Berl.), 37: 297-314.

Harris, C.E., Knowlton, F.F., 2001. Differential responses of coyotes to novel stimuli in familiar and unfamiliar settings. Canadian Journal of Zoology, 79, 2005-2013.

Harkins, K.M., Keinath, D., Ben-David, M., 2019. It's a trap: Optimizing detection of rare small mammals. PLoS ONE 14(3): e0213201. https://doi.org/10.1371/journal.pone.0213201

Hayward, G.F., Phillipson, J., 1979. Community structure and functional role of small mammals in ecosystems. In: Stoddart D.M. (eds) Ecology of small mammals. Springer, Dordrecht

Henttonen, H., Oksanen, T., Jortikka, A., Haukisalmi, V., 1987. How much do weasels shape microtine cycles in the northern Fennoscandian taiga? Oikos 50: 353- 365.

Hilborn, R., J. A. Redfield, Krebs, C. J., 1976. On the reliability of enumeration for mark and re-capture census of voles. Canadian Journal of Zoology 54:1019-1024.

Hickey, J.R., Sollmann, R. 2018. A new mark-recapture approach for abundance estimation of social species. PLoS ONE 13(12):e0208726.https://doi.org/10.1371/journal.pone.0208726

Hooten, M.B., Hobbs, N.T., 2015. A Guide to bayesian model selection for ecologists. Ecol Monogr. ap;85: 3-28

Iknayan, K., Tingley, M.W., Furnas, B., Beissinger, S.R., 2014. Detecting diversity: emerging methods to estimate species diversity. Trends in Ecology \& Evolution 29(2). DOI: 10.1016/j.tree.2013.10012

Ims, R. A., Fuglei, E. 2005. Trophic interaction cycles in tundra ecosystems and the impact of climate change. - BioScience 55: 311-322.

Jareño, D., Viñuela, J., Luque-Larena, J.J., Arroyo, L., Arroyo, B., Mougeot, F. A., 2014. Comparison of methods for estimating common vole (Microtus arvalis) abundance in agricultural habitats. Ecol Indic;36: 111-119 
Jareño, D., Viñuela, J., Luque-Larena, J.J., Arroyo, L., Arroyo, B., Mougeot, F., 2015. Factors associated with the colonization of agricultural areas by common voles Microtus arvalis in NW Spain. Biol Invasions;17: 23152327.

Jacob, J., Hempel, N., 2003. Effects of farming practices on spatial behaviour of common voles. J Ethol;21: 45-50.

Jacob, J., 2003. Short-term effects of farming practices on populations of common voles. Agric. Ecosyst. Environ., 95, pp. 321-325

Jacob, J., Manson, P., Barfknecht, R., Fredricks, T., 2014. Common vole (Microtus arvalis) ecology and management: Implications for risk assessment of plant protection products. Pest Manag Sci;70: 869-878. pmid:24293354

Jacob, J., Brown, J.S., 2000. Microhabitat use, giving-up densities and temporal activity as short- and long-term anti-predator behaviors in Common voles. Oikos;91: 131-138.

Jacob, J., Tkadlec, E., 2010. Rodent outbreaks in Europe: dynamics and damage. In: Singleton G, Belmain S, Brown P, Hard B, editors. Rodent outbreaks: Ecology and Impacts. The International Rice Research Institute(IRRI);pp.207-

224.Available: http://books.irri.org/9789712202575_content.pdf

Jackson, D.M., Trayhurn, P., Speakman, J.R., 2001. Associations between energetics and over-winter survival in the short-tailed field vole Microtus agrestis. J Anim Ecol;70: 633-640.

Jensen, T. S., Olsen, K., Hansen, T.S., Vedel-Smith, C., 2012. Home range and movements of the field vole Microtus agrestis in an organic agro ecosystem. Mammalian Biology. http://www.orgprints.org/19073/

Jiménez, J. 2017. Modelos jerárquicos bayesianos aplicados al seguimiento de fauna (PhD Thesis) CSIC-UCLM - Instituto de Investigación en Recursos Cinegéticos (IREC). Ciudad Real. Available at: http://hdl.handle.net/10578/15277

Jiménez, J., Nuñez-Arjona, J.C., Rueda, C., González, L.M., GarcíaDomínguez, F., Muñoz-Igualada, J., et al., 2017. Estimating carnivore community structures. Sci Rep;7: 41036-. pmid:28120871

Jung, T.S., O'Donovan, K.S., 2005. Mortality of Deer mice, Peromyscus maniculatus, in wire mesh live-traps: A cautionary note. Can FieldNaturalist.;119:445-

446.http://www.canadianfieldnaturalist.ca/index.php/cfn/article/viewFile/1 $60 / 160$ 
Jung, T.S., 2016. Comparative efficacy of Longworth, Sherman, and Ugglan live-traps for capturing small mammals in Nearctic boreal. Mammal Research; 61(1):57-64.

Kikkawa, J., 1964. Movement, activity and distribution of small rodents Clethrionomys glareolus and Apodemus syluaticus in woodland. Journal of Animal Ecology 33: 259-299.

Karanth, K.U., Nichols, J.D, 1998. Estimation of tiger densities in India using photographic captures and recaptures. Ecology, 79(8), pp. 2852-2862

Kellner, K. F., \& Swihart, R. K., 2014. Accounting for imperfect detection in ecology: a quantitative review. Plos one, 9(10), e111436. doi:10.1371/journal.pone.0111436

Krebs, C., Boonstra, R., 1984. Trappability estimates for mark-recapture data. Canadian Journal of Zoology 62(12):2440-2444. DOI: 10.1139/z84-360

Kéry, M. \& Schmidt, B.R., 2008. Imperfect detection and its consequences for monitoring for conservation. Community Ecology, 9, 207- 216.

Kie, J. G., Matthiopoulos, J., Fieberg, J., Powell, R. A., Cagnacci, F., Mitchell, M. S. Moorcroft, P. R. 2010. The home-range concept: are traditional estimators still relevant with modern telemetry technology?. Philosophical transactions of the Royal Society of London. Series B, Biological sciences, 365(1550), 2221-2231. doi:10.1098/rstb.2010.0093

Kie J. G. Bowyer R. T.1999. Sexual segregation in white-tailed deer: densitydependent changes in use of space, habitat selection, and dietary niche. Journal of Mammalogy 80: 1004-1020.

Ketz, A.C., Johnson, T.L., Monello, R.J., Mack, J.A., George, J.L., Kraft, B.R., Wild, M.A., Hooten, M.B., Hobbs, N.T., 2018. Estimating abundance of an open population with an $\mathrm{N}$-mixture model using auxiliary data on animal movements. Ecological Applications 28(3): 816-825. https://doi.org/10.1002/eap.1692

Kery, M., Gardner, B., Stoeckle, T., Weber, D. \& Royle, J.A., 2011. Use of spatial capture-recapture modelling and DNA data to estimate densities of elusive animals. Conserv. Biol. 25, 356-364.

Kéry, M., Schaub, M., 2012. Bayesian population analysis using WinBUGS. A hierarchical perspective, Academic Press/Elsevier.ISBN: 9780123870209

Klemola, T., Korpimäki, E., Koivula, M., 2002. Rate of population change in voles from different phases of the population cycle. Oikos,Vol. 96, No. 2, pp. 291-298. https://doi.org/10.1034/j.1600-0706.2002.960211.x 
Korpimäki, E., Brown, P.R., Jacob, J., Pech, R.P., 2004. The puzzles of population cycles and outbreaks of small mammals solved? BioScience, 54, 1071-1079

Kosztolányi, A., Barta, Z., Küpper, C. \& Székely, T., 2011. Persistence of an extreme male-biased adult sex ratio in a natural population of polyandrous bird. Journal of Evolutionary Biology. 24(8):1842-6. doi: 10.1111/j.1420-9101.2011.02305.x

Kokko, H., Jennions, M.D., 2008. Parental investment, sexual selection and sex ratios. J Evol Biol;21 (4):919-48. doi: 10.1111/j.1420-9101.2008.01540.x

Koskela, E., Mappes, T. and Ylonen, H., 1997. Territorial behaviour and reproductive success ofbank voleClethrionomys glareolusfemales.J. Anim. Ecol.66, 341-349.

Krebs, C.J., Bilodeau, F., D. Reid, D., Gauthier, G., Kenney, A.J., Gilbert, S., Duchesne, D., Wilson, D.J., 2012. Are lemming winter nest counts a good index of population density? Journal of Mammalogy 93:87-92.

Krebs, C.J, Boonstra, R., Gilbert, S., Reid, D., Kenney, A.J., Hofer, E.J., 2011. Density estimation for small mammals from live trapping grids: rodents in northern Canada. J Mammal;92: 974-981

Krebs, C.J., Reid, D.G., Kenney, A.J., Gilbert, S., 2011. Fluctuations in lemming populations in north Yukon, Canada, 2007-2010. Canadian Journal of Zoology 89:297- 306.

Krebs, C.J., 2013. Population fluctuations in rodents. The University of Chicago Press, Chicago. University of Chicago Press, Chicago, Illinois, ix +306 pp. ISBN 978-0-226-01035-9

Krebs, C.J., 2014. In Chapter 2: Estimating Abundance and Density: MarkRecapture Techniques. Ecological Methodology, 3rd ed. Addison-Wesley Educational Publishers, Inc.

Krebs, C.J., 2011. Of lemmings and snowshoe hares: the ecology of northern Canada. - Proc. R. Soc. B 278: 481-489.

Krebs, C.J., Boonstra, R., Kenney, A.J., 1995. Population dynamics of the collared lemming and the tundra vole at Pearce Point, Northwest Territories, Canada. Oecologia 103: 481- 489.

Krebs, C.J., 1999. Ecological Methodology. 2nd Edition, Benjamin Cummings, Menlo Park, $620 \mathrm{p}$.

Krebs, C.J., 1999. Chapter 5 - Estimating Abundance: Line Transects, in: Ecological Methodology.

Krebs, C.J., Boonstra, R., 1984. Trappability estimates for mark-recapture data. Can J Zool;62: 2440-2444. 
Krebs, C.J. 1978. Ecology. The Experimental Analysis of Distribution and Abundance. Second Edition. Harper and Row, New York. 678 pp

Kuo, L., Mallick, B., 1998. Variable selection for regression models. Sankhyā Indian J Stat Ser B;60: 65-81.

Kvarnemo, C., Ahnesjö, I., 1996. The dynamics of operational sex ratios and competition for mates. Trends in Ecology and Evolution, vol. 11, issue 10, pp. 404-408

Lambin, X., N. G. Yoccoz. 1998. The effect of populationkin structure on nestling survival in Townsend's voles,Mi-crotus townsendii. Journal of Animal Ecology67:1-16.

Lambin, X., Petty, S.J., Mackinnon, J.L., 2000. Cyclic dynamics in field vole populations and generalist predation. J. Anim. Ecol. 69, 106-118. https://doi.org/10.1046/j.1365-2656.2000.00380.x

Lambin, X., Bretagnolle, V., Yoccoz, N.G., 2006. Vole population cycles in northern and southern Europe: Is there a need for different explanations for single pattern? J Anim Ecol;75: 340-349. pmid:16637987

Lambin, X., MacKinnon, J., 1997. The relative efficiency of two commercial livetraps for small. J. Zool., Lond. 242,400404. https://zs/publications.onlinelibrary.wiley.com/doi/pdf/

Lambin X., 1997. Home range shifts by breeding female Townsend's voles (Microtus townsendii): A test of the territory bequeathal hypothesis. Behavioral Ecology and Sociobiology 40(6):363-372, Doi: $10.1007 / \mathrm{s} 002650050352$

Lambin, X., J. Aars, S. B. Piertney, 2001. Dispersal, intraspecific competition, kin competition and kin facilitation: a review of the empirical evidence. Pages 110-122 in J. Clobert, E. Danchin, A. A. Dhondt, and J. D. Nichols, eds. Dispersal. Oxford University Press, Oxford

Leibold, M.A., McPeek, M.A., 2006. Coexistence of the niche and neutral perspectives in community ecology. Ecology. 87(6):1399-410.

Le Galliard, J.F, Fitze, P.S, Ferrière, R., Clobert, J., 2005. Sex ratio bias, male aggression, and population collapse in lizards. Proceedings of the National Academy of Sciences, 102 (50) 18231-18236; doi: 10.1073/pnas.0505172102

Lee, A.M., Saether, B.-E., Engen, S., 2011. Demographic stochasticity, Allee effects, and extinction: the influence of mating system and sex ratio. Am. Nat. 177: 301-313.

Lindberg, M. S. 2012. A review of designs for capture-mark-recapture indiscrete time. Journal of Ornithology 152:355-370

Liro, A. 1974. Renewal of burrows by the common vole as the indicator of its 
numbers. Acta Theriologica 19:259-272.

Lima, S.L., Bednekoff, P.A., 1999. Temporal variation in danger drives antipredatory behaviour: the predation risk allocation hypothesis. Am Nat 153:649-659

Lisická, L., Losik, J., Zejda, J., Heroldová, M., Nesvadbová, J., Tkadlec, E., 2007. Measurement error in a burrow index to monitor relative population size in the common vole. Folia Zool.-56 (2): 169-176

Liker, A., Freckleton RP, Székely T., 2013. The evolution of sex roles in birds is related to adult sex ratio. Nat Commun; 4:1587. doi: 10.1038/ncomms2600.

Loughran, M.F.E., 2006. Social organization of female field voles Microtus agrestis in a population in Southern England. Acta Theologica 51: 233242.

López-Bao, J.V., Godinho, R., Pacheco, C., Lema, F.J., García, E., Llaneza, L., Palacios, V., Jiménez, J., 2018. Toward reliable population estimates of wolves by combining spatial capture-recapture models and non-invasive DNA monitoring. Scientific Reports 8, 2177

Luque-Larena, J.J., Mougeot, F., Viñuela, J., Jareño, D., Arroyo, L., Lambin, X., Arroyo, B., 2013. Recent large-scale range expansion and eruption of common vole (Microtus arvalis) outbreaks in NW Spain Basic and Applied Ecology in press. DOI: 10.1016/j.baae.2013.04.006

Luque-Larena, J.J., Mougeot, F., Roig, D.V., Lambin, X., Rodríguez-Pastor, R., Rodríguez-Valín, E., et al., 2015. Tularemia outbreaks and common vole (Microtus arvalis) irruptive population dynamics in Northwestern Spain, 1997-2014. Vector-Borne Zoonotic Dis.;15: 568-570. pmid:26333034

Luque-Larena, J.J., Mougeot, F., Arroyo, B., Dolors, Vidal, M., RodríguezPastor, R., Escudero, R., et al., 2017. Irruptive mammal host populations shape Tularemia epidemiology. Plos Pathog;13: e1006622. pmid:29145508

Luque-Larena, J.J., López, P., Gosálbez, J., 2001. Scent matching modulates space use and agonistic behaviour between male snow voles, Chionomys nivalis. Anim Behav;62: 1089-1095.

Luque-Larena, J.J., López, P., Gosálbez, J., 2002. Microhabitat use by the snow vole Chionomys nivalis in alpine environments reflects rockdwelling preferences. Can J Zool;80: 36-41.

Luque-Larena, J.J., López, P., Gosálbez, J., 2002. Relative dominance affects use of scent-marked areas in male snow voles Chionomys nivalis. Ethology;108: 273-285.

Lyra-Jorge, M.C., Ciochet, G., Pivello, V.R., Meirelles, S.T., 2008. Comparing methods for sampling large- and medium-sized mammals: camera traps 
and track plots. Eur J Wildl Res 54: 739-744. DOI 10.1007/s10344-0080205-8

Mazurkiewicz, M., Rajska, E., 1998. Spatial behavior and population dynamics of woodland rodents. Acta theriologica 43(2):137-161. DOI: 10.4098/AT.arch.98-11

Madison, D.M., 1978. Movement Indicators of Reproductive Events among Female Meadow Voles as Revealed by Radiotelemetry, Journal of Mammalogy, Volume 59, Issue 4, Pages 835-843, https://doi.org/10.2307/1380147

Martin, G., 2003. The role of small ground-foraging mammals in topsoil health and biodiversity: implications to management and restoration. Ecol Manag Restor 4(2):114 - 119.

Mayle, B.A., Peace A.J., Gill, R.M.A.,1999. How Many Deer? A Field Guide to Estimating Deer Population Size. Edinburgh, Forestry Commission: 96.

Massei, G., Coats, J., Lambert, M.S., Pietravalle, S., Gill, R., Cowan, D., 2018. Camera traps and activity signs to estimate wild boar density and derive abundance indices. Pest Manag Sci. 74(4):853-860. doi: $10.1002 / p s .4763$

Mackin-Rogalska., 1981. Spatial structure of rodent populations co-occurring in different crop fields. Pol. Ecol. Stud.7, pp. 213-227

Mazerolle, M.J., Larissa L. Bailey, William L. Kendall, J. Andrew Royle, Sarah J. Converse, James D. Nichols., 2007. "Making Great Leaps Forward: Accounting for Detectability in Herpetological Field Studies," Journal of Herpetology 41(4), 672-689. https://doi.org/10.1670/07-061.1

Mauvisseau, Q, Parrondo, M., Fernández, M.P., García, L., Martínez, J.L,. García-Vazquez, E., Borrell Pichs, Y., 2017. On the way for detecting and quantifying elusive species in the sea: The Octopus vulgaris case study. Fisheries Research, 191: 41-48. DOI: 10.1016/j.fishres.2017.02.023

Martin, J., Edwards, H.H., Burgess, M.A., Percival, H.F., Fagan, D.E., Gardner, B.E, et al., 2012. Estimating distribution of hidden objects with drones: From tennis balls to manatees. PLoS One. 7: 1-8. pmid:22761712

McCullough D. R. Hirth D. H. Newhouse S. J.. 1989. Resource partitioning between sexes in white-tailed deer. Journal of Wildlife Management $53: 277-283$.

MacKenzie, D.I., Nichols, J.D., Royle, J.A., Pollock, K.H., Bailey, L.L., Hines, J.E., 2006. Occupancy Estimation and Modeling: Inferring Patterns and Dynamics of Species Occurrence. Burlington, MA, USA: Academic Press; 
Mowat G, Strobeck C., 2000. Estimating population size of grizzly bears using hair capture, DNA profiling, and mark-recapture analysis. J. Wildlife Manage.;64:183-193.

McCarthy, M.A., Moore, J.L., Morris, W.K., Parris, M.P., Garrard, G.E., Vesk, P.A. et al. 2013. The influence of abundance on detectability. Oikos, 122, $717-726$

McKnight, D.T., Ligon, D.B., 2017. Correcting for unequal catchability in sex ratio and population size estimates. PLoSONE 12(8): e0184101. https://doi.org/10.1371/journal.pone.0184101

Miñarro, M., Montiel, C., Dapena, E., 2012. Vole pests in apple orchards: use of presence signs to estimate the abundance of Arvicola terrestris cantabriae and Microtus lusitanicus. J Pest Sci. DOI 10.1007/s10340012-0438-x

Morin, D.J., Fuller, A.K., Royle, J.A., Sutherland, C., 2017. Model-based estimators of density and connectivity to inform conservation of spatially structured populations. Ecosphere;8: e01623.

Moore, S.L, Wilson, K., 2002. Parasites as a viability cost of sexual selection in natural populations of mammals. Science;297(5589):2015-8.

Mougeot, F., Lambin, X., Rodríguez-Pastor, R., Romairone, J., Luque-Larena, J.J., 2019. Numerical response of a mammalian specialist predator to multiple prey dynamics in Mediterranean farmlands. Ecology. Online: https://doi.org/10.1002/ecy.2776

Montgomery, W.I., 1980. Mortality of small rodents captured in live-traps. Acta Theriol(Warsz);25:277294. http://rcin.org.pl/Content/10645/BI002_2613_ Cz-40-2_Acta-T25-nr22-277-294_o.pdf

Muneza, A., Linde, D.W., Montgonery, R., Dickman, A.J., Roloff, G., MacDonald, D., Fennessy, J., 2017. Examining disease prevalence for species of conservation concern using non-invasive spatial capturerecapture techniques. Journal of Applied Ecology. DOI: 10.1111/13652664.12796

Navara, J., 2018. Choosing Sexes: Mechanisms and Adaptive Patterns of Sex Allocation in Vertebrates. Springer-Verlag. ISBN: 9783319712697

Nichols, J.D., Pollock, K. H., 1983. Estimation methodology in contemporary small mammal capture-recapture studies. Journal of Mammalogy 64: 253-260.

Nichols, J.D., Karanth, K.U., 2002. Statistical concepts: estimating absolute densities of tigers using capture-recapture sampling. Pages 123-138 in K.U Karanth and Nichols, editors. Monitoring tigers and their prey: a manual for researchers, managers and conservacionist in tropical Asia. Centre of wildlife studies, Bangalore. 
Noguerales, V., Traba, J., Mata, C., Morales, B., 2015. Winter habitat selection and partitioning in two sympatric farmland small mammals: Apodemus sylvaticus and Mus spretus. Revue d'Ecologie; 70: 7082.http://hdl.handle.net/2042/56311

O'Brien, J., 1994. Voles. Pages B-177-B-182 in S. Hygnstrom, R., Timm, and G. Larsen, editors. Prevention and control of wildlife damage. Cooperative Extension Division, University of Nebraska, Lincoln, Nebraska, USA.

O'Connell, A.F., Nichols, J.D., Karanth K.U., 2011. Camera traps in animal ecology: methods and analyses. Tokyo: Springer

O'Connor KM, Nathan LR, Liberati MR, Tingley MW, Vokoun JC, Rittenhouse TAG, 2017. Camera trap arrays improve detection probability of wildlife: Investigating study design considerations using an empirical dataset. PLoS ONE 12(4): e0175684. https://doi.org/10.1371/journal. pone. 0175684

Ostfeld, R. S. 1990. The ecology of territoriality in small mammals. Trends Ecol. Evol., 5: 411-415.

Otis, D.L., Burnham, K.P., White, G.C., 1978. Anderson DR. Statistical inference from capture data on closed animal populations. Wildl Monogr;62: 3-135.

Ostfeld, R.S., Pugh, S.R., Seamon, J.O., Tamarin, R.H., 1988. Space use and reproductive success in a population of Meadow Voles. J Anim Ecol;57: 385-394.

Paz, A., Jareño, D., Arroyo, L., Viñuela, J., Arroyo, B., Mougeot, F., LuqueLarena, J.J., Fargallo, J.A., 2013. Avian predators as a biological control system of common vole (Microtus arvalis) populations in north-western Spain: experimental set-up and preliminary results. Pest Manage. Sci. 69, 444-450.

Palombit, R.A., 2012: in The Evolution of Primate Societies, J. C. Mitani, J. Call, P. Kappeler, R. A. Palombit, J. B. Silk, Eds. (Univ. of Chicago Press, Chicago, 2012), pp. 432-468.

Palomo, L. J., Gisbert, J., 2002. Atlas de los Mamíferos Terrestres de España. Dirección General de Conservación de la Naturaleza-SECEM -SECEMU, Madrid. 564 pp.

Parmenter, R.R., Yates, T.L., Anderson, D.R., Burnham, K.P., Dunnum, J.L., Franklin, A.B., Friggens, M.T., Lubow, B.C., Miller, M., Olson, G.S., Parmenter, C.A., Pollard, J., Rexstad, E., Shenk, T.M., Stanley, T.R., White, G.C., 2003. Small- mammal density estimation: a field comparison of grid- based vs. web-based density estimators. Ecol. Monogr. 73, 1-26. 
Pearson, D.E., Hierro, J.L., Chiuffo, M., Villarreal, D., 2014. Rodent seed predation as a biotic filter influencing exotic plant abundance and distribution. Biological Invasions, 16, 1185-1196.

Pinot, A., Barraquand, F., Tedesco, E., Lecoustre, V., Bretagnolle, V., Gauffre, B., 2016. Density-dependent reproduction causes winter crashes in a common vole population. Popul Ecol;58: 395-405.

Piggot, M.P., Taylor, A.C., 2003. Remote collection of animal DNA and its applications in conservation management and understanding the population biology of rare and cryptic species. Wildlife Research 30(1) 1 13

Pita, R., Beja, P., Mira, A., 2007. Spatial population structure of the Cabrera vole in Mediterranean farmland: The relative role of patch and matrix effects. Biological Conservation, 134: 383-392.

Pita, R., Mira, A., Beja, P., 2010. Spatial segregation of two vole species (Arvicola sapidus and Microtus cabrerae) within habitat patches in a highly fragmented farmland landscape. Eur. J. Wildl. Res; 56: 651-662.

Pikula, J., Treml, F., Beklova, M., Holesovska, Z., Pikulova, J., 2002. Geographic information systems in epidemiology - ecology of common vole and distribution of natural foci of Tularaemia. Acta Veterinaria Brno; 71:379-387

Plummer, M., Best, N., Cowles, K., Vines, K., 2006. CODA: convergence diagnosis and output analysis for MCMC. $R$ News;6: 7-11. Available: https://cran.r-project.org/doc/Rnews/Rnews_20061.pdf\#page $=7$

Pollock, K. H., Nichols, J.D., Simons, T.R., Farnsworth, G.L., Bailey, L.L., Suaer, R., 2002. Large scale wildlife monitoring studies: statistical methods for design and analysis. Environmetrics. Vol.13, Issue 2, pp.105-119.

Pollock, K.H., Nichols, J.D., Hines, J. E., Brownie, C., 1990. Statistical inference for capture-recapture experiments. Wildlife Monographs 107.

Pollock, K.H., 1982. A capture-recapture design robust to unequal probability of capture. The Journal of Wildlife Management. Vol. 46, No. 3, pp. 752-757

Power, M., 1992. Top-down and bottom-up forces in food webs: do plants have primacy. Ecology, 73, 733-746

Pugh, S., Johnson, S., Tamarin, R., 2003. Voles. Pages 349-370 in G. Feldhamer, B. Thompson, and J. Chapman, editors. Wild Mammals of North America. The Johns Hopkins University Press, Baltimore, Maryland. 
Preston, C.R., 1990. Distribution of raptor foraging in relation to prey biomass and habitat structure. Condor 92, 107-112.

Quéré, J.P., Raoul, F., Giraudoux, P., Delattre, P., 2000. An index method applicable at landscape scale to estimate relative population densities of the common vole (Microtus arvalis). Revue d'Écologie, Terre et Vie $55: 25-32$

Rankin, D.J. Dieckmann, U, Kokko, H., 2011. Sexual conflict and the tragedy of the commons. Am. Nat., 177 (6), pp. 780-791

Radchuk, V., Ims, R.A., Andreassen, H.P., 2016. From individuals to population cycles: the role of extrinsic and intrinsic factors in rodent populations. Ecology, Vol.97, Issue 3, pp. 720-732

Rees, S.G., Goodenough, A.E, Hart, A.G., Stafford, R., 2011. Testing the effectiveness of capture mark recapture population estimation techniques using a computer simulation with known population size. Ecological Modelling 222, 3291-3294.

Réale, D., Reader, S.M., Sol, D., McDougall, P.T., Dingemanse, N.J., 2007. Integrating animal temperament within ecology and evolution. Biol Rev;82: 291-318. pmid:17437562

Roff, D.A. 2002. Life History Evolution. Sinauer Associates, Sunderland, MA. DOI: 10.1016/B978-0-12-384719-5.00087-3. ISBN: 0878937560

Rodríguez-Pastor, R., Escudero, R., Vidal, D., Mougeot, F., Arroyo, B., Lambin, X., Vila-Cor, A.M., Rodriguez-Moreno, I., Anda, P., Luque-Larena, J.J., 2017. Density-dependent prevalence of Francisella tularensis in fluctuating vole populations, Northwestern Spain. Emerg Infect Dis;23: 1377-1379. pmid:28726608

Rodríguez-Pastor, R., Luque-Larena, J.J., Lambin, X., Mougeot, F., 2016. "Living on the edge": The role of field margins for common vole (Microtus arvalis) populations in recently colonised Mediterranean farmland. Agric Ecosyst Environ;231: 206-217.

Rodríguez-Pastor, R., Escudero, R., Lambin, X., Vidal, M.D., Gil, H., Jado, I., Rodríguez-Vargas, M., Luque-Larena, J.J., Mougeot, F., 2019. Zoonotic pathogens in fluctuating common vole (Microtus arvalis) populations: occurrence and dynamics. Parasitology, 146(3), 389-398. https://doi.org/10.1017/S0031182018001543

Romairone, J., Jiménez, J., Luque-Larena, J.J., Mougeot, F., 2018. Spatial capture-recapture design and modelling for the study of small mammals. PLoS ONE 13(6): e0198766.

Rosenberg, D.K., Anthony, R.G., 1993. Differences in trapping mortality rates of northern flying squirrels. Can J Zool;71(3): 660-663. 
Royle, J.A., Kéry, M., Guélat, J., 2011. Spatial capture-recapture models for search-encounter data. Vol. 2, Issue 6, pp. 602-611. https://doi.org/10.1111/j.2041-210X.2011.00116.x

Royle, J.A., Chandler, R.B., Sun, C.C., Fuller, A.K., 2013. Integrating resource selection information with spatial capture-recapture. O'Hara RB, editor. Methods Ecol Evol;4: 520-530. https://doi.org/10.1111/2041-210X.12039

Royle, J.A., Chandler, R.B., Sollmann, R., Gardner, B., 2014. Spatial capturerecapture. Waltham, Massachusetts: Elsevier, Academic Press; https://doi.org/10.1016/B978-0-12-405939-9.00020-7

Royle, J.A., Young, K.A., 2008. Hierarchical model for spatial capture-recapture data;89: 2281-2289.

Royle, J.A., Fuller, A.K., Sutherland, C., 2017. Unifying population and landscape ecology with spatial capture-recapture. Ecography (Cop); 112.

Royle, J.A., Karanth, K.U., Gopalaswamy, A.M., Kumar, N.S., 2009. Bayesian inference in camera trapping studies for a class of spatial capturerecapture models. Ecology;90: 3233-3244. pmid:19967878

Royle, J.A., Converse, S.J., 2014. Hierarchical spatial capture-recapture models: modelling population density in stratified populations. Methods Ecol Evol;5: 37-43.

Rota, C.T., Fletcher, R.J., Evans, J.M, Hutto, R.L., 2011. Does accounting for imperfect detection improve species distribution models? Ecography (Cop). 34: 659-670

Sabino-Marques, H., Ferreira, C.M., Paupério, J., Costa, P., Barbosa, S., Encarnação, C., Alpizar Jara, R., Alves, P.C., Searle, J.B., Mira, A., Beja, P., Pita, R., 2018. Combining genetic non-invasive sampling with spatially explicit capture-recapture models for density estimation of a patchily distributed small mammal. Eur. J. Wildl.Res. 64. https://doi.org/10.1007/s10344-018-1206-x

Sandell, M., Agrell, J., Erlinge, S., Nelson, J., 1991. Adult philopatry and dispersal in the field vole Microtus agrestis. Oecologia 86: 153-158

Seber, G.A.F., 1982. The Estimation of Animal Abundance and Related Parameters. Blackburn Press. ISBN: 1930665555

Seber, G., 1986. A review of estimating animal abundance. Biometrics;42: 267292. Available: http://www.jstor.org/stable/2531049

Sinclair, A., Krebs, C.J., 2002. Complex numerical responses to top-down and bottom-up processes in vertebrate populations. Philosophical Transactions of the Royal Society of London, 357, 1221. 
Sollmann, R., Furtado, M.M., Gardner, B., Hofer, H., Jácomo, A.T.A., Tôrres, N.M., et al., 2011. Improving density estimates for elusive carnivores: Accounting for sex-specific detection and movements using spatial capture-recapture models for jaguars in central Brazil. Biol Conserv. Elsevier Ltd;144: 1017-1024.

Sollmann, R., Gardner, B., Belant, J.L., 2012. How does spatial study design influence density estimates from spatial capture-recapture models? PLoS One;7: e34575. pmid:22539949

Sollmann, R., Gardner, B., Parsons, A.W., Stocking, J.J., McClintock, B.T., Simons, T.R, Pollock, K-H., O'Connell, A.F., 2013. A spatial mark-resight model augmented with telemetry data. Vol. 94, Issue 3, pp. 553-559. https://doi.org/10.1890/12-1256.1

Skalski, J., K. Ryding., J. Millspaugh., 2005. Analysis of Sex, Age, and Count Data. Wildlife Demography, $1^{\text {st }}$ Ed. Academic Press. ISBN: 9781493300068

Székely, T., Weissing, F. J., Komdeur, J., 2014. Adult sex ratio variation: implications for breeding system evolution. J. Evol. Biol., 27: 1500-1512. doi:10.1111/jeb.12415

Schmidt, B.R., Pellet, J., 2010: Quantifying abundance: counts, detection probabilities, and estimates. In: Amphibian Ecology and Conservation, p. 465-479. Dodd, K.C., Ed., Oxford, Oxford University Press.

Schmitz, O.J., Beckerman, A.P., O’Brien, K.M., 1997. Behaviorally mediated trophic cascades: effects of predation risk on food webs. Ecology 78:13881399

Schwarz, C.J., Seber, G.A.F., 1999. Estimating Animal Abundance: Review III. Statistical Science, $14, \quad 427-456$. http://dx.doi.org/10.1214/ss/1009212521

Sheridan, M., Tamarin, R.H., 1988. Space use, longevity, and reproductive success in meadow voles. Behav Ecol Sociobiol; 22: 85-90.

Schaub M., Royle J. A.. 2014. Estimating true instead of apparent survival using spatial Cormack-Jolly-Seber models. Methods Ecol. Evol. 5:1316-1326

Shannon, G., Lewis, J.S., Gerber, B.D., 2014. Recommended survey designs for occupancy modelling using motion-activated cameras: insights from empirical wildlife data. PeerJ. 2: e532. pmid:25210658

Spiegelhalter, D., Thomas, A., Best, N., Way, R., 2003. WinBUGS User Manual. Components; 2, 1-60. http://www.mrc-bsu.cam.ac.uk/bugs 
Stamps, J., Krishnan, V.V., 1997. Sexual bimaturation and sexual size dimorphism in animals with asymptotic growth after maturity. Evolutionary Ecology 11: 21-39

Stenseth, N.C., Ims, R.A., 1993. Population dynamics of lemmings: temporal and spatial variation-an introduction. In: Stenseth NC and Ims RA, editors. The Biology of Lemmings. Linnean Society, London. pp. 61-96.

Stephens, R.B., Anderson, E.M., 2014. Effects of trap type on small mammal richness, diversity, and mortality. Wildl Soc Bull;38: 619-627.

Steiner, J. J., Gavin, W. E., Mueller-Warrant, G. W., Griffith, S. M., Whittaker, G. W., Banowetz, G. M., 2007. Conservation practices in western Oregon perennial grass seed systems. II. Impacts on gray-tailed vole activity. Agronomy Journal 99:537-542.

Stoddart, D.M., Smith, P.A., 1986. Recognition of odour-induced bias in the livetrapping of Apodemus sylvaticus. Oikos;46: 194-199.

Stradiotto, A., Francesca Cagnacci, Richard Delahay, Silvia Tioli, Luis Nieder, Annapaola Rizzoli,. 2009. Spatial Organization of the Yellow-Necked Mouse: Effects of Density and Resource Availability, Journal of Mammalogy, Volume 90, Issue 3, Pages 704-714, https://doi.org/10.1644/08-MAMM-A-120R1.1

Stromgren, E.J., 2008. Improving Live trapping methods for Shrews (Sorex spp.) [Internet]. The University of British Columbia (Vancouver). Available: https://open.library.ubc.ca/cIRcle/collections/ubctheses/24/item s/1.0066780\#downloadfiles

Stromgren, E.J., Sullivan, T.P., 2014. Influence of pitfall versus Longworth livetraps, bait addition, and drift fences on trap success and mortality of shrews. Acta Theriol (Warsz);59: 203-210.

Schaub, M., Royle, J.A., 2014. Estimating true instead of apparent survival using spatial Cormack-Jolly-Seber models. Methods Ecol Evol;5: 13161326.

Sun, C.C., Fuller, A.K., Hare, M.P., Hurst, J.E., 2017. Evaluating population expansion of black bears using spatial capture-recapture. J Wildl Manage;81: 814-823.

Sun, C.C., Fuller, A.K., Royle, J.A., 2014. Trap configuration and spacing influences parameter estimates in spatial capture-recapture models. PLoS One;9: e88025. pmid:24505361

Sutherland, C.S., Elston, D.A., Lambin, X., 2014. A demographic, spatially explicit patch occupancy model of metapopulation dynamics and persistence. Ecology;95: 3149-3160. 
Sutherland, C., Royle, J.A., Linden, D., 2018. oSCR: Multi-Session SexStructured Spatial Capture-Recapture Models. R package version 0.41.0. https://rdrr.io/github/jaroyle/oSCR/

Sutherland, C., Fuller, A.K., Royle, A.J., 2015. Modelling non-Euclidean movement and landscape connectivity in highly structured ecological networks. Methods in Ecology and Evolution. Vol. 6, Issue, 2. Pp. 169177. https://doi.org/10.1111/2041-210X.12316

Tapper, S., 1979. The Effect of Fluctuating Vole Numbers (Microtus agrestis) on a Population of Weasels (Mustela nivalis) on Farmland. Journal of Animal Ecology. Vol. 48, No. 2, pp. 603-617. DOI: 10.2307/4182

Tattersall, F.H., Macdonald, D.W., 2003. Wood mice in the arable ecosystem. Pages 82-96 in: F.H. Tattsersall \& W.J. Manley (eds.) Conservation and Conflict: Mammals and Farming in Britain. Linnean Society Occasional Publications, Westbury Publishing, West Yorkshire, UK.

Tanaka, R., 1963. On the problem of trap-response types of small mammal populations. Res Popul Ecol (Kyoto);5: 139-146.

Taylor, A.K., Hellström, P., Angerbjörn, A., 2011. Effects of trap density and duration on vole abundance indices. Ann Zool Fennici; 48: 45-55.

Terraube, J., Arroyo, B., Madders, M., Mougeot, F., 2011. Diet specialisation and foraging efficiency under fluctuating vole abundance: A comparison between generalist and specialist avian predators. Oikos 120, 234-244. https://doi.org/10.1111/j.1600-0706.2010.18554.x

Tellería, J., 2004. Métodos de Censos en Vertebrados Terrestres, Dpto. Biología. Animal I (Zoología de Vertebrados). Fac. de Biología, Univ. Complutense. Madrid-España.

Available: https://www.ucm.es/zooantropo/zoologia-aplicada-vertebrados-terrestres

Thomson, D., Conroy, E.G., Cooch, M.J., 2009. Modeling demographic processes in marked populations. Thomson DL, Cooch EG, Conroy MJ, editors. Modeling Demographic Processes in Marked Populations, Environmental and Ecological Statistics. Boston, MA: Springer US; https://doi.org/10.1007/978-0-387-78151-8

Thompson, W.L., White, G.C., Gowan, C., 1998: Monitoring Vertebrate Populations. San Diego, Academic Press. ISBN: 9780126889604

Tkadlec, E., Stenseth, N.C., 2001. A new geographical gradient in vole population dynamics. Proc R Soc B Biol Sci;268: 1547-1552. pmid:11487400

Traviani, A., Aldama, J.J., Delibes, M., 1993. Red fox capture locations in relation to home range boundaries. Mammalia 57, 448-451 
Trolle, M., Marc Kéry, 2003. Estimation of Ocelot Density in the Pantanal Using Capture-Recapture Analysis of Camera-Trapping Data, Journal of Mammalogy, Volume 84, Issue 2, Pages 607-614, https://doi.org/10.1644/1545-1542(2003)084<0607:EOODIT>2.0.CO;2

Van der Hoek, Y., Zuckerberg, B., Manne, L.L., 2015. Application of habitat thresholds in conservation: Considerations, limitations, and future directions. Global Ecology and Conservation, 3, 736743. https://doi.org/10.1016/j.gecco.2015.03.010

Valente, A.M., Fonseca, C., Marques, T.A., Santos, J.P, Rodrigues, R., Torres, R.T, 2014. Living on the edge: Roe deer (Capreolus capreolus) density in the margins of its geographical range. PLoS ONE 9(2): e88459. DOI: https://doi.org/10.1371/journal.pone.0088459

Veran, S., Beissinger, S.R., 2009. Demographic origins of skewed operational and adult sex ratios: perturbation analyses of two-sex models. Ecology Letters. 12(2):129-43. doi: 10.1111/j.1461-0248.2008.01268.x.

Verdolin, J.L., 2006. Meta-analysis of foraging and predation risk trade-offs in terrestrial systems. Behav Ecol Sociobiol;60: 457-464.

Verhagen, R., Leirs, H., Verheyen, W., 2000. Demography of Clethrionomys glareolus in Belgium. Pol. J. Ecol; 48:113-123

Vidal, D., Alzaga, V., Luque-Larena, J.J., Mateo, R., Arroyo, L., Viñuela, J., 2009. Possible interaction between a rodenticide treatment and a pathogen in common vole (Microtus arvalis) during a population peak. Sci. Total Environ. 408(2) :267-271. https://doi.org/10.1016/j.scitotenv.2009.10.001

Virgós, E., Casanovas, J., 1999. Badger Meles meles sett site selection in low density Mediterranean areas of central Spain. Acta Theriologica, 44 (2): 173-182.

Warren, C.C., Veech, J.A., Weckerly, F.W., O'Donnell, L., Ott, J.R., 2013. Detection Heterogeneity and Abundance Estimation in Populations of Golden-Cheeked Warblers (Setophaga chrysoparia). The Auk 130(4), https://doi.org/10.1525/auk.2013.13022

Waterman, J., 2007. Male mating strategies in rodents In: Wolff JO, Sherman PW, eds. Rodent societies: an ecological \& evolutionary perspective. Chicago: The University of Chicago Press; pp. 27-41.

Wedekind, C., Evanno, G., Szekely, T., Pompini, M., Darbellay, O. et al., 2013. Persistent Unequal Sex Ratio in a Population of Grayling (Salmonidae) and Possible Role of Temperature Increase. Conservation Biology, Wiley, 27 (1), pp.229 - 234.

White, G.C., D. R. Andersen, K. P. Burnham, and D. L. Otis., 1982. Capturerecapture and removal methods for sampling closed populations. Los Alamos National Laboratory Report LA-8787-NERP,. Los Alamos. New Mexico, USA. pp 
Wheeler, P., 2008. Effects of sheep grazing on abundance and predators of field vole (Microtus agrestis) in upland Britain. Agriculture, Ecosystems \& Environment, 123(1-3) pp. 49-55.

Williams, B.K., Nichols, J.D., Conroy, M.J., 2002. Analysis and management of animal populations. Academic Press, California. Pp: 242, 257-332.

Wilson, G.J., Delahay, R.J., 2001. A review of the methods to estimate the abundance of terrestrial carnivores using field signs and observation. Wildlife Res 28:151-164

Wilson, K.R., Anderson, D.R., 1985. Evaluation of two density estimators of small mammal population size. J Mammal;66: 13-21.

Willson, J.D., Winne, C.T., Todd, B.D., 2011. Ecological and methodological factors affecting detectability and population estimation in elusive species. J Wildl Manage; 75: 36-45.

Wiewel, A.S., Clark, W.R., Sovada, M.A., 2007. Assessing small mammal abundance with track- tube indices and mark-recapture population estimates. Journal of Mammalogy 88:250-260.

Witmer, G.W., 2005. Wildlife population monitoring: some practical considerations. Wildl. Res. 32, 259-263.

Wobeser, G.A., 2007. Disease in Wild Animals. Investigation and Management. Springer. ISBN 978-3-540-48978-8

Wolff, J.O., Cicirello, D.M., 1990. Mobility versus territoriallity: alternative reproductive strategies in white-footed mice. Anim Behav;39: 1222-1224.

Wolff, J.O., 2007. Social biology of rodents. Integr Zool;2(4):193-204. doi: 10.1111/j.1749-4877.2007.00062.x.

Wolff, J.O., 1993. Why Are Female Small Mammals Territorial? Oikos, 68(2), 364-370. doi:10.2307/3544853

Wood, S., 2017. Generalized Additive Models. An Introduction with R, Second Edition. New York. https://doi.org/10.1201/9781315370279. ISBN: 9781315370279

Ylönen, H., Mappes, T., Viitala, J., 1990. Different demography of friends and strangers: an experiment on the impact of kinship and familiarity in Clethrionomys glareolus. Oecologia;83: 333-337. pmid:28313003

Ylönen, H., Jacob, J., Kotler, B.P., 2003. Trappability of rodent in single-capture and multiple-capture traps in arid and open environments: Why don't Ugglan traps work?. Ann. Zool. Fennici; 40: 537-541. ISSN: 0003-455X

Ylönen, H., Ronkainen, H., 1994. Breeding suppression in the bank vole as antipredatory adaptation in a predictable environment. Evol Ecol 8:658-666 
Yip, P.S., Xi, L., Chao, A., Hwang, W.H., 2000. Estimating the population size with a behavioral response in capture-recapture experiment. Environmental and Ecological Statistics 7(4):405-414. DOI: 10.1023/A:1026575616678

Yoccoz, N.G., Steen, H., Ims, R.A., Stenseth, N.C. 1993. Estimating demographic parameters: an updated methodological survey. In Biology of lemmings. Edited by N.C. Stenseth and R.A. Ims. Academic Press, London. Pp. 565-587.

Zhang, Y., Zhang, Z., Liu, J., 2003. Burrowing rodents as ecosystem engineers: the ecology and management of plateau zokors Myospalax fontanierii in alpine meadow ecosystems on the Tibetan Plateau. Mammals review. Vol.33, Issue, 3-4, pp. 284-294. https://doi.org/10.1046/j.13652907.2003.00020.x 


\section{Acknownledgments}

This is the part of the thesis that everyone loves to read (and if anyone denies that, they are a liar!). The acknowledgments are also the part which requires the most time and thought, especially when it the author's desire not to leave anyone unmentioned, especially since, in a direct or indirect way, all of you have participated in this thesis. This thesis becomes a reality with the kind support and help of many people. I would like to extend my sincere thanks to all of them. Therefore, I will take my time and dedication to express my thanks to all of you.

I would like to express my deepest appreciation and gratitude to my Supervisors Dr. Juan José Luque Larena and Dr. François R. Mougeot, for giving me the opportunity to fulfill my dream of being a Ph.D. in Ecology, and for your support throughout the years. But overall for imparting and sharing your expertise and knowledge that allowed me to achieve my aim successfully. Without your guidance and persistent help this thesis would not have been possible.

Also, I would like to thank to the most fantastic ecologists I have ever met, such as José "Pepe" Jiménez, Beatriz "Bea" Arroyo and Xavier Lambin. Pepe, one of the biggest "cracks" of Bayesian statistics (with love, one of those crazy lovers of numbers, and extensive and incomprehensible mathematical equations). Many thanks Pepe for teaching me to be more orderly and meticulous with the data (otherwise, models would never have run!). Also, for being available 24/7: when I had a simple problem and I contacted you via mail, you responded back to me in milliseconds. Bea, a renowned researcher whom I 
did not hesitate for a second when asking her if she wanted to provide me comments and advice in one of the chapters. I would be remised if I did not also mention her wonderful hospitality when letting me stay in her home (the homemade hummus was delicious, Bea!). As for Lambin, thanks a million for your valuable comments and advice in the development of my chapters. For me, it is an honor to have participated in and be published together in "Ecology" - the magnificent weasel paper. It is a pleasure to know you as a person (outside of academics or Professor status) and to have spent time "waiting's" trying to spot brown bears and iberian wolves in Montaña Palentina (Palencia, Spain). I am very grateful to all of you, my friends.

Many thanks and appreciations to all my $\mathrm{Ph}$. D colleagues of the "Escuela Técnica Superior de Ingenierías Agrarias" (ETSIIAA, Palencia), from the University of Aberdeen (Scotland) and other friends (my lifelong friends who already know who you are!). Special mention to Ruth Rodríguez-Pastor who never hesitated to share her time (either in the office or in the field) with me. It was like having another Supervisor (she already is/will be a fantastic researcher!). She was always available and willing to perfectly answer and clarify my infinite doubts. Deon Roos, a magnificent training bio-statistician who helped me in many tasks such as field work in the enclosure, correcting my written English and gave me some valuables comments and advices. Also, Silvia Herrero who was ever-willing to help me in field when I needed it and Sara García for all these great moments capturing voles in the field. Without forgetting, all my colleagues whom I shared with so many good moments at the University (having coffee or having lunch in the cafeteria) and some adventures in the mountains (Montaña Palentina). Many thanks to Cristina Zamora, Nicolás 
Cattaneo, José Riofrío, Sara Uzquiano, Olaya Mediavilla, Jordán Muñóz, Jorge Aldea, Carmen Romeralo, Ana Martin, María Casado, Juan C. Vinagrero, Elena Muñoz, Asdrubal Flores, Jorge Martín, etc., for all the marvelous and special moments disconnecting from the world, as well as the delightful companionship that was an omnipresent with you all.

My sincere thanks to my friend Fernando Jubete. Firstly for being one of my mentors in the field for a few years now, with whom I have been able to learn a lot of things about birds, the capture and identification of butterflies, monitoring wild cats in the mountains, carrying out routine censuses in the Cerrato Palentino, the capture and marking of owls, etc., and now, in this case, the world of small mammals like voles. On the other hand, I want to highlight your unending availability for everything, and having the doors of your house is routinely open. Not to mention, the enviable visits to your house in Montaña Palentina that you were always gracious enough to offer. Thanks, Fer.

Many thanks to the members of ITACyL who participated in this thesis, especially Constantino "Tinín" Caminero-Saldaña and María "Charo" Rosario for helping in the enclosure and collecting data that has been used in a chapter of this thesis but also for your valuable comments and suggestions in the development of it.

I would like to offer thanks to all of the people I met during my stays at IREC ("Instituto de Investigación en Recursos Cinegéticos") as well as all the researchers, technicians and other Ph.D. students with whom made me feel very welcome. 
I am really grateful to all my friends who have supported me in the development of this thesis during these last 3.5 years. You all have endured my lengthy discussions of voles (...and surprisingly you are all still my friends!!). You have all guided and even advised me to focus on the thesis and not go to the mountains every weekend - many thanks to Cristina Zamora, Elena Muñoz, Natalia Diez, Daniel Sanchez, Álvaro Giraldo.

My greatest gratitude to my little family for their steadfast and permanent support and for believing in me. Special thanks to my mum, this thesis is dedicated to her. She worked tirelessly on the daunting task of doing capturemark-recapture of a highly reproductive pest species, yet throughout it you were unwavering with your gracious help. The high quality of the data gathered and used in this thesis has only been possible because of the inestimable help of my mother who was monitoring with me for several weeks, months, etc. Not once did she complain despite working with rodents (which she does not like too much!) and being under adverse weather conditions in summer, autumn or winter. Sandwiches and a hot/cold drink were an ever-present with her (a mother's care). Thanks.

This thesis was funded by research projects ECOVOLE (CGL201235348) and ECOTULA (CGL2015-66962-C2-1-R), financed by Ministerio de Economía y Competitividad (MINECO), Spain. I held a research contract (PEJ2014A-05890_IEJ15/15; "Contrato de empleo joven e implantación de la garantía juvenil I+D+I", 2015-2017) at the University of Valladolid (UVA). I had a grant for $\mathrm{Ph}$. D students at the University of Valladolid (MOVILIDAD DOCTORANDOS UVa 2019). 
Finally, I want to thank the external reviewers of this thesis and the reviewers of the published manuscripts for the constructive remarks and suggestions. 


\section{Appendix}

9.1. Appendix 1

Peer-reviewed articles:

Romairone, J., Jiménez, J., Luque-Larena, J.J., Mougeot, F., 2018. Spatial capture-recapture design and modelling for the study of small mammals. PLoS ONE 13(6): e0198766. https://doi.org/10.1371/journal.pone.0198766 


\section{S1 Supporting Information}

Variables contributing to monthly variation in the VAS performance: $g_{0}$ and $\sigma$

To explore an influence of vole behavior, $g_{0}$ and $\sigma$ obtained from the SCR models were included in subsequent models with a total of 20 month (vs. 18 months), therefore, including those months that were excluded because of extra-compensatory heterogeneity (January 2017 and April 2017). Including or excluding these months did not affect the results or conclusions.

Table 1. SCR model estimates of vole behavior ( $g_{0}$ and $\sigma$ ) when applied all the months (20 months) of the study.

\begin{tabular}{ccccc}
\hline Model & F & $\boldsymbol{p}$ & ${\text { Adjusted- } \mathbf{r}^{2}}$ & Multiple- $^{2}$ \\
\hline BU_CL_DR $\sim \log (\mathrm{D})+\log (\sigma)$ & $\mathrm{F}_{2,17}=16.48$ & 0.0001 & 0.61 & 0.65 \\
BU_CL_DR $\sim \log (\mathrm{D})+\log \left(\mathrm{g}_{0}\right)$ & $\mathrm{F}_{2,17}=32.14$ & $<.001$ & 0.76 & 0.79 \\
\hline
\end{tabular}




\section{S1 Supporting information}

This supplementary material shows the results of the analyses repeated using 17 months of data (i.e. including the two months - January 2017 and April of 2017- that were excluded from analyses in the main text because of evidence of extra-compensatory heterogeneity). Note that including or excluding these two months did not affect conclusions.

\section{Association between $A_{S R} R_{C M R}$ and $A S R_{S T S}$}

I ran SCR models using a total of 17 monthly CMR sessions. The association $A_{\text {SRMR }}$ and ASR $R_{S T S}$ was positive but only marginally significant $\left(F_{1,15}=3.80, p=\right.$ 0.07 , Multiple- $r^{2}=0.202 ;$ Adjusted- $r^{2}=0.149 ;$ Figure S1)

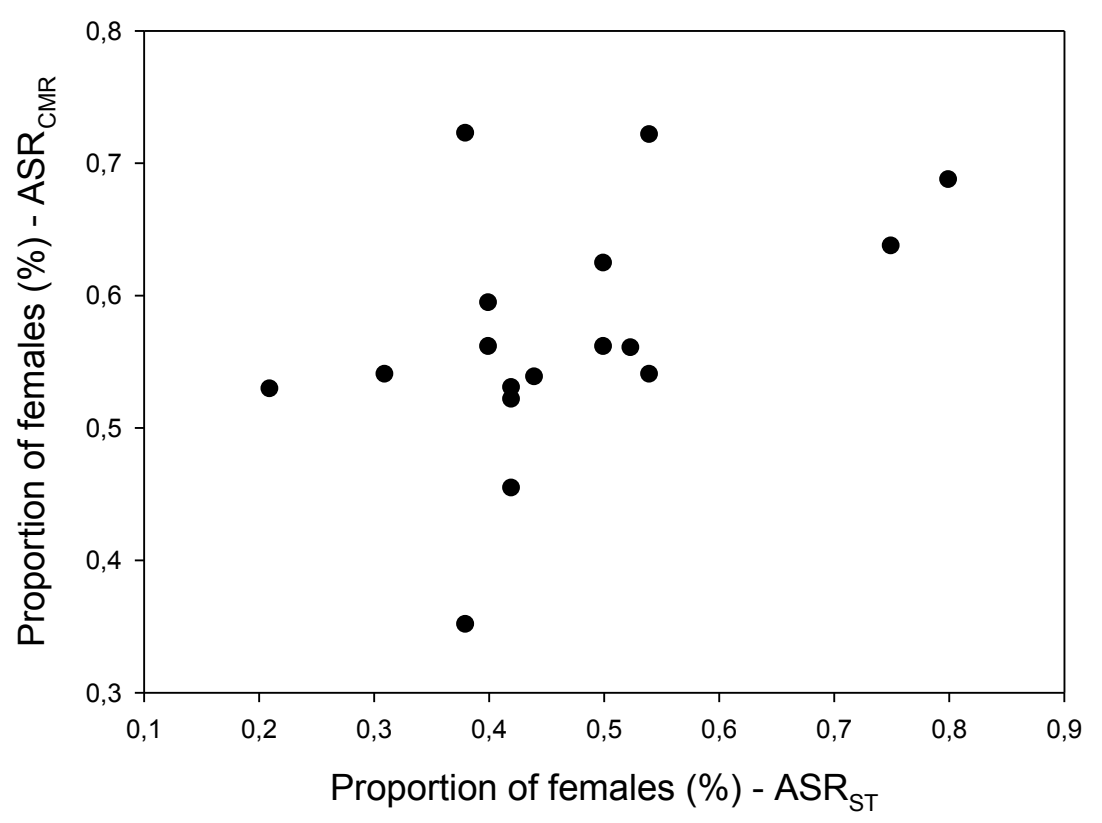

Figure S1. Relationship between the proportion of females (\%) obtained in SCR modelling (CMR) and the proportion of females (\%) obtained by STS method ( $\mathrm{n}=17$ months). 


\section{Model outputs and other parameters}

a) Sigma ( $\sigma$ ) and basal probability detection ( $\left.g_{0}\right)$

Table 1. Parameters $\sigma$ (movement) and $g_{0}$ (basal probability of detection) of male and female voles (data including all 17 months).

\begin{tabular}{lcccc}
\hline \multicolumn{1}{c}{ Model parameters } & \multicolumn{2}{c}{ Females } & \multicolumn{2}{c}{ Males } \\
\cline { 2 - 5 } & $\operatorname{Min}$ & $\operatorname{Max}$ & Min & Max \\
$\boldsymbol{\sigma}$ (movement) & $3.50 \pm 0.37$ & $14.53 \pm 1.18$ & $6.25 \pm 0.38$ & $12.45 \pm 1.27$ \\
$\mathbf{g}_{\mathbf{0}}$ (basal probability detection) & $0.05 \pm 0.01$ & $0.42 \pm 0.10$ & $0.04 \pm 0.02$ & $0.45 \pm 0.13$ \\
\hline
\end{tabular}

b) Vole density (D) and vole behavior

b.1) Univariate analysis

Table 2. Results of the univariate analyses ( $n=17$ months) to explain the residuals from the association between $A \mathrm{AR}_{\mathrm{CMR}}$ and $\mathrm{ASR}_{\mathrm{STS}}$ (square root-transformed). Significant variables are highlighted in bold.

\begin{tabular}{lcccc}
\hline Models & $\mathbf{F}$ & $\boldsymbol{p}$ & Multiple-r $^{2}$ & Adjusted-r $^{2}$ \\
\hline ASR $_{\mathrm{CMR}} \sim \mathrm{ASR}_{\mathrm{ST}}+$ Density & $\mathrm{F}_{2,14}=3.04$ & 0.05 & $\mathbf{0 . 3 3 6}$ & $\mathbf{0 . 2 4 1}$ \\
ASR $_{\mathrm{CMR}} \sim \mathrm{ASR}_{\mathrm{ST}}+\sigma_{\text {females }}$ & $\mathrm{F}_{2,14}=4.12$ & 0.03 & $\mathbf{0 . 3 7 0}$ & $\mathbf{0 . 2 8 0}$ \\
ASR $_{\mathrm{CMR}} \sim \mathrm{ASR}_{\mathrm{ST}}+\sigma$ males & $\mathrm{F}_{2,14}=3.40$ & 0.06 & 0.327 & 0.231 \\
ASR $_{\mathrm{CMR}} \sim \mathrm{ASR}_{\mathrm{ST}}+\mathrm{g}_{\text {o females }}$ & $\mathrm{F}_{2,14}=2.12$ & 0.15 & 0.232 & 0.123 \\
ASR $_{\mathrm{CMR}} \sim \mathrm{ASR}_{\mathrm{ST}}+\mathrm{g}_{0 \text { males }}$ & $\mathrm{F}_{2,14}=5.42$ & 0.01 & $\mathbf{0 . 4 3 6}$ & $\mathbf{0 . 3 5 6}$ \\
\hline
\end{tabular}

b.2) Multivariate analysis

Table 3. Results of the multivariate analyses ( $n=17$ months) to explain the residuals from the association between $A_{S R_{C M R}}$ and $A_{S R_{S T S}}$ (square root-transformed).

\begin{tabular}{ccccc}
\hline Model & $\mathbf{F}$ & $\boldsymbol{p}$ & $\begin{array}{c}\text { Multiple } \\
-\mathbf{r}^{2}\end{array}$ & $\begin{array}{c}\text { Adjusted } \\
-\mathbf{r}^{2}\end{array}$ \\
\hline $\begin{array}{l}\mathrm{ASR}_{\mathrm{CMR}} \sim \mathrm{ASR}_{\mathrm{ST}}+\text { season }+\sigma \\
\text { females }+\mathrm{g}_{0} \text { males }\end{array}$ & $\mathrm{F}_{6,10}=10.31$ & 0.0008 & $\mathbf{0 . 8 6 9}$ & $\mathbf{0 . 7 7 7}$ \\
\hline
\end{tabular}


c) Variations in parameters $\sigma$ and go according to season, reproduction and density

Table 4. Results of the GLMs explaining variations in the $\sigma$ parameter of females and males. Significant variables are highlighted in bold.

\begin{tabular}{llcccc}
\hline \multirow{2}{*}{ Models } & F & $\boldsymbol{p}$ & $\begin{array}{c}\text { Multiple } \\
-\mathbf{r}^{2}\end{array}$ & $\begin{array}{c}\text { Adjusted } \\
-\mathbf{r}^{2}\end{array}$ \\
\hline \multirow{3}{*}{$\boldsymbol{\sigma}$ females } & + season & $\mathrm{F}_{3,17}=0.64$ & 0.59 & 0.102 & -0.056 \\
& + density & $\mathrm{F}_{1,15}=0.26$ & 0.61 & 0.017 & -0.048 \\
& + reproduction & $\mathrm{F}_{1,19}=8.61$ & 0.008 & $\mathbf{0 . 3 1 2}$ & $\mathbf{0 . 2 7 5}$ \\
& & & & & \\
\multirow{6}{*}{$\boldsymbol{\sigma}$ males } & & & & \\
& + season & $\mathrm{F}_{3,17}=0.94$ & 0.44 & 0.142 & -0.008 \\
& + density & $\mathrm{F}_{1,15}=0.74$ & 0.40 & 0.047 & -0.016 \\
& + reproduction & $\mathrm{F}_{1,19}=8.64$ & 0.008 & $\mathbf{0 . 3 1 2}$ & $\mathbf{0 . 2 7 6}$ \\
\hline
\end{tabular}

Table 5. Results of the GLMs explaining variations in the go parameter of females and males.

\begin{tabular}{llcccc}
\hline & Models & F & $\boldsymbol{p}$ & $\begin{array}{c}\text { Multiple } \\
-\mathbf{r}^{2}\end{array}$ & $\begin{array}{c}\text { Adjusted } \\
-\mathbf{r}^{2}\end{array}$ \\
\hline \multirow{3}{*}{$\mathbf{g}_{0}$ females } & + season & $\mathrm{F}_{3,17}=1.11$ & 0.37 & 0.164 & 0.017 \\
& + density & $\mathrm{F}_{1,15}=0.07$ & 0.78 & 0.005 & -0.061 \\
& + reproduction & $\mathrm{F}_{1,19}=0.00$ & 0.95 & 0.000 & -0.052 \\
& & & & & \\
\multirow{3}{*}{$\mathbf{g}_{0}$ males } & & & & \\
& + season & $\mathrm{F}_{3,17}=1.35$ & 0.29 & 0.193 & 0.050 \\
& + density & $\mathrm{F}_{1,15}=2.85$ & 0.11 & 0.159 & 0.103 \\
& + reproduction & $\mathrm{F}_{1,19}=0.11$ & 0.73 & 0.006 & -0.046 \\
\hline
\end{tabular}




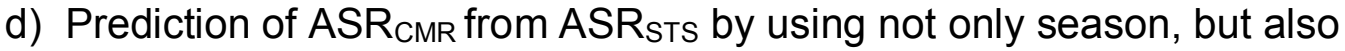
information on the proportion of reproductive females

Table 6. Table of coefficients of a linear best model with season and reproduction as best predictors ( $n=17$ months).

\begin{tabular}{lcccc}
\hline \multicolumn{1}{c}{ Factor } & Estimate & SE & $\mathbf{t}$ & $\mathbf{P}$ \\
\hline Intercept & 0.38055 & 0.07043 & 5.403 & 0.000216 \\
ASRst & 0.41524 & 0.12926 & 3.213 & 0.008268 \\
Season_spring & 0.01859 & 0.05971 & 0.311 & 0.761316 \\
Season_summer & -0.11337 & 0.04094 & -2.770 & 0.018241 \\
Season_winter & -0.06284 & 0.04770 & -1.317 & 0.214532 \\
Reproduction & 0.13384 & 0.08773 & 1.526 & 0.155323 \\
\hline
\end{tabular}

*ASRST = proportion of females captured in the population.

** Reproduction $=$ proportion of reproductive females

\section{S2 Supporting Information}

Number of vole recaptures in the study period

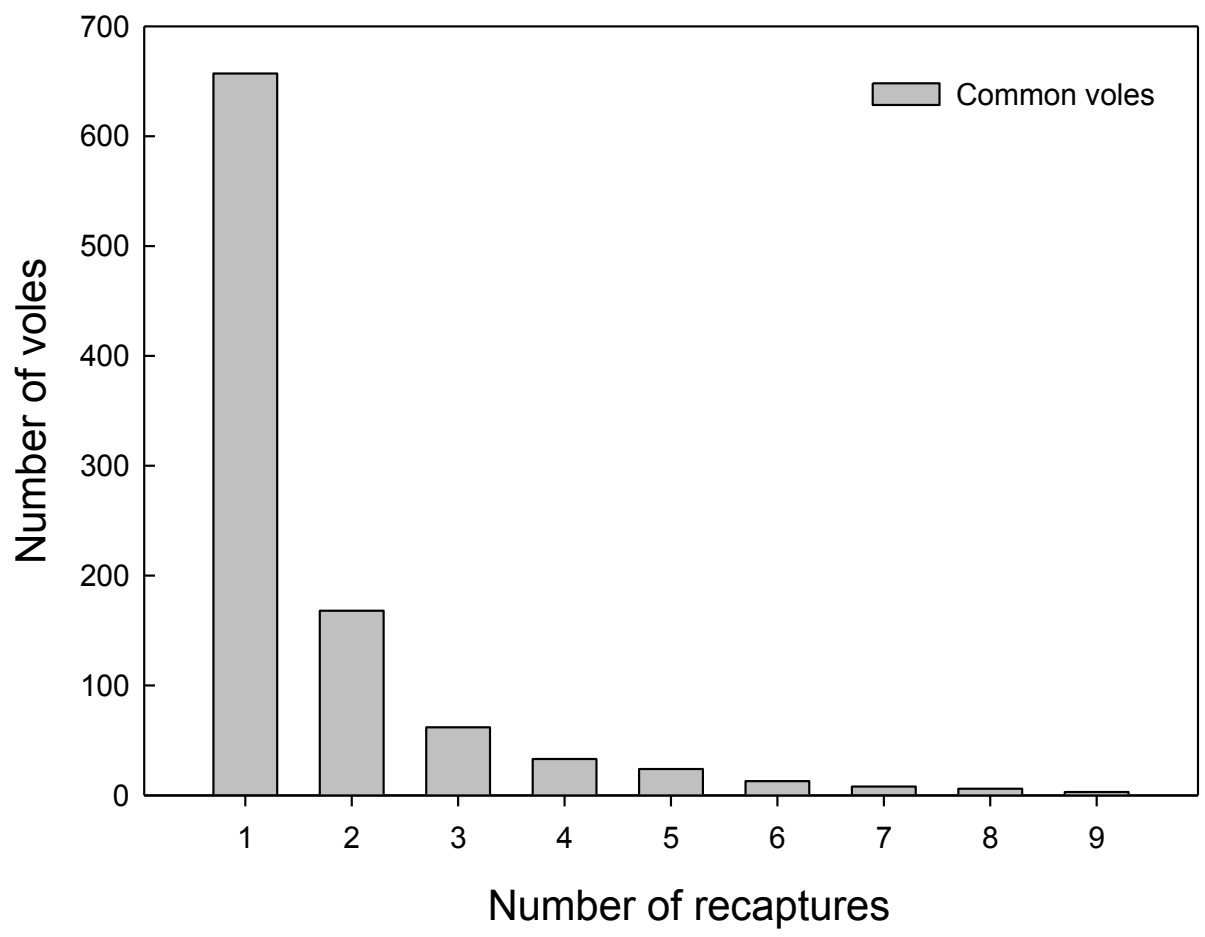

Figure 1. Number of the maximum times of common voles recaptured in the plot in the study period (May 2016 - July 2018). 